\title{
Some New Aspects of the Boyer Reaction
}

\author{
Rupak Chakraborty, Veronica Franz, Ghanashyam Bez, Dipali Vasadia, Chenchu Popuri \\ and Cong-Gui Zhao*
}

Supplementary Material 


\section{Supporting Information}

Table of Contents

General Methods

Page

S2

General Procedure for Acetal Formation

S3

Procedure for the preparation of macrocyclic ligand $\mathbf{3}$

S4

Procedure for the preparation of propargylamine

S5

General Procedure for the Boyer reaction of aliphatic aldehydes

And 2-Aryl-2-azidoethanol

S6

$\begin{array}{lr}\text { NMR Data for New Compounds } & \text { S7 }\end{array}$

$\begin{array}{lr}\text { NMR Spectra of New Compounds } & \text { S18 }\end{array}$

HPLC Analysis of Optically Active 3-Oxazolines $\quad$ S82 


\section{General Methods}

All solvents and reagents, unless individually specified, were purchased from Aldrich Chemical Company and were used without further purification. Dichloromethane was dried over calcium hydride and distilled prior to use. Benzene was purchased from Alfa Aesar and used without further purification. HPLC grade methanol (EM Science) was used directly.

Silica gel 60 (230-400 Mesh ASTM, particle size 0.04-0.063 mm) for column chromatography was purchased from EM Science. Thin layer chromatography (TLC) was performed using TLC aluminium sheets precoated with Merck silica gel $60 \mathrm{~F}_{254}$.

${ }^{1} \mathrm{H}$ and ${ }^{13} \mathrm{C}$ NMR spectra were recorded on a Varian $500 \mathrm{MHz}$ NMR spectrophotometer using $\mathrm{CDCl}_{3}$ as solvent with TMS as an internal standard.

Enantiomeric excess was determined by using a Shimadzu HPLC with a Chiralcel OD-H column and hexane/iso-propanol as eluent. 


\section{General Procedure for Acetal Formation}

Caution: Diazides are potential explosives and should be handled with care and all necessary protective measures should be taken during the reaction.

To an oven dried $15 \mathrm{~mL}$ round-bottom flask equipped with a magnetic stirring bar was added 0.3 $\mathrm{mmol}$ of aldehyde, $0.3 \mathrm{mmol}$ of 2-aryl-2-azidoethanol, $5 \mathrm{~mL}$ of dry dichloromethane and $5 \mathrm{~mol} \%$ of $\mathrm{Cu}(\mathrm{OTf})_{2}$. The reaction mixture was refluxed overnight. The reaction mixture was diluted with dichloromethane $(25 \mathrm{~mL})$ and neutralized with $10 \%$ aqueous $\mathrm{NaHCO}_{3}$ solution $(10 \mathrm{~mL})$. Organic layer was separated, dried (over $\mathrm{MgSO}_{4}$ ) and evaporated under reduced pressure. The crude product was purified by flash chromatography, using $2 \%$ ethyl acetate in hexane as eluent to afford the desired acetal. 


\section{Procedure for the Preparation of Macrocyclic Ligand 3}

A mixture of the acetal $1 \mathrm{a}(537 \mathrm{mg}, 1.28 \mathrm{mmol}), 5 \% \mathrm{Pd}-\mathrm{C}(27 \mathrm{mg}, 5 \% \mathrm{~mol})$ in $20 \mathrm{~mL}$ methanol was hydrogenated under stirring overnight by using hydrogen balloon. After hydrogenation, the reaction mixture was filtered. The solvent was evaporated under reduced pressure. The crude product was purified by column chromatography over silica gel using $10 \% \mathrm{MeOH}$ in $\mathrm{CH}_{2} \mathrm{Cl}_{2}$ to get the diamine $\mathbf{2 a}$ in $318 \mathrm{mg}(71 \%)$.

Diamine 2a: ${ }^{1} \mathrm{H}$ NMR $\left(500 \mathrm{MHz}, \mathrm{CDCl}_{3}\right) \delta 0.81(\mathrm{~s}, 3 \mathrm{H}), 1.19(\mathrm{~m}, 6 \mathrm{H}), 1.53(\mathrm{~m}, 2 \mathrm{H}), 3.60(\mathrm{~m}, 2 \mathrm{H})$, $3.80(\mathrm{~m}, 2 \mathrm{H}), 4.17(\mathrm{brs}, 2 \mathrm{H}), 4.47(\mathrm{~s}, 1 \mathrm{H}), 5.26(\mathrm{br}, 4 \mathrm{H}), 7.49(\mathrm{~m}, 10 \mathrm{H}) \mathrm{ppm}$.

${ }^{13} \mathrm{C}$ NMR $\left(125 \mathrm{MHz}, \mathrm{CDCl}_{3}\right) \delta 139.7,139.4,128.8,128.7,128.2,128.1,128.0,127.5,127.5$, $127.4,103.8,70.7,70.2,55.7,55.5,33.2,31.7,24.4,22.8,14.2 \mathrm{ppm}$.

A mixture of diamine $\mathbf{2 a}(494 \mathrm{mg}, 1.38 \mathrm{mmol})$ and 2,6-pyridine dicarboxaldehyde (206 $\mathrm{mg}, 1.52$ $\mathrm{mmol}$ ) in $110 \mathrm{~mL}$ methanol was refluxed for $2 \mathrm{~h}$ at an oil bath temperature of $75^{\circ} \mathrm{C}$. Afterwards methanol was removed under reduced pressure to get a solid product. The crude product was further purified by washing two times with $15 \mathrm{~mL}$ boiling methanol. The yield of the final macrocyclic ligand $\mathbf{3}$ is $579 \mathrm{mg}(92 \%)$.

Ligand 3: ${ }^{1} \mathrm{H}$ NMR $\left(500 \mathrm{MHz}, \mathrm{CDCl}_{3}\right) \delta 0.87(\mathrm{~m}, 3 \mathrm{H}), 1.11(\mathrm{~m}, 6 \mathrm{H}), 1.54(\mathrm{~m}, 2 \mathrm{H}), 3.83(\mathrm{~m}, 4 \mathrm{H})$, $4.60(\mathrm{~m}, 3 \mathrm{H}), 7.44(\mathrm{~m}, 11 \mathrm{H}), 8.08(\mathrm{~m}, 2 \mathrm{H}), 8.51$ (brs, 2H) ppm.

${ }^{13} \mathrm{C}$ NMR $\left(125 \mathrm{MHz}, \mathrm{CDCl}_{3}\right) \delta 158.9,142.6,142.5,142.4,142.4,137.6,128.6,128.6,128.6$, 127.7, 127.6, 127.1, 127.1, 127.08, 119.3, 104.0, 72.06, 71.9, 64.5, 55.9, 55.8, 33.5, 31.8, 24.5, 22.7, $14.2 \mathrm{ppm}$. 


\section{Procedure for the Preparation of Propargylamine}

The ligand 3 (9.1 mg, $0.02 \mathrm{mmol}, 10 \mathrm{~mol} \%$ ), copper(I)triflate toluene complex (10 mg, $0.02 \mathrm{mmol}$, $10 \mathrm{~mol} \%$ ) were stirred at room temperature in $0.5 \mathrm{~mL}$ benzene under nitrogen atmosphere for 15 min. N-Benzylideneaniline (36 mg, $0.2 \mathrm{mmol})$, phenyl acetylene $(38 \mathrm{mg}, 0.22 \mathrm{mmol})$ in $1 \mathrm{~mL}$ benzene were added at room temperature under nitrogen. The reaction mixture was heated to $60{ }^{\circ} \mathrm{C}$ oil bath temperature and stirred overnight $(20 \mathrm{~h})$. After cooling, the mixture was extracted with ether $(3 \times 10 \mathrm{~mL})$ and washed with water $(10 \mathrm{~mL})$ and dried over anhydrous $\mathrm{MgSO}_{4}$. The solvent was evaporated under reduced pressure. The crude product was purified by column chromatography over silica gel by using $1 \%$ ethyl acetate in hexane as eluent to afford the propargylamine $^{7 \mathrm{c}}(43 \mathrm{mg}, 75 \%)$. 
General Procedure for the Boyer reaction of aliphatic aldehydes with 2-aryl-2-azidoethanol

A mixture of the aldehyde $(0.3 \mathrm{mmol})$, 2-aryl-2-azidoethanol $(0.3 \mathrm{mmol})$ in $5 \mathrm{~mL}$ dry dichloromethane and $\mathrm{BF}_{3} \cdot \mathrm{Et}_{2} \mathrm{O}$ (equivalents as mentioned in table $2 \& 3$ ) were stirred at specific temperature (Table $2 \& 3$ ). Afterwards the reaction mixture was diluted with dichloromethane (25 $\mathrm{mL}$ ) and neutralized with $10 \%$ aqueous $\mathrm{NaHCO}_{3}$ solution $(10 \mathrm{~mL})$. Organic layer was separated, dried (over $\mathrm{MgSO}_{4}$ ) and evaporated under reduced pressure. The crude product was purified by column chromatography to get the products 4 (eluted in 2\% EtOAc-hexane), 5 (eluted in 10\% EtOAc-hexane) and $\mathbf{6}$ (eluted in methanol). 


\section{Characterization of acetals in Table 1}

\section{Entry 1}

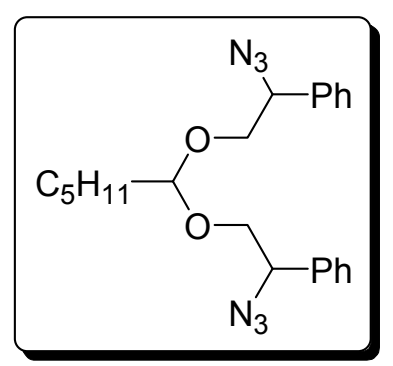

${ }^{1} \mathrm{H}$ NMR (500 MHz, CDCl 3$) \delta 0.80$ (brs, 3H), 1.24 (brs, 6H), $1.55(\mathrm{~m}, 2 \mathrm{H}), 3.60(\mathrm{~m}, 4 \mathrm{H}), 4.56(\mathrm{~m}$, $3 \mathrm{H}), 7.25(\mathrm{~m}, 10 \mathrm{H}) \mathrm{ppm}$.

${ }^{13} \mathrm{C}$ NMR $\left(125 \mathrm{MHz}, \mathrm{CDCl}_{3}\right) \delta 136.9,136.9,129.0,129.0,128.9,128.7,128.7,128.7,127.4$, $127.3,127.3,127.3,103.7,69.3,68.6,65.8,65.5,32.8,31.7,24.4,22.7,14.2 \mathrm{ppm}$.

\section{Entry 2}

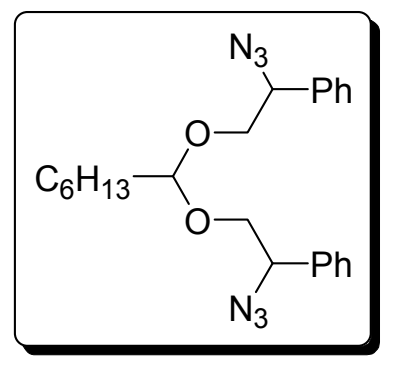

${ }^{1} \mathrm{H}$ NMR $\left(500 \mathrm{MHz}, \mathrm{CDCl}_{3}\right) \delta 0.88$ (brs, 3H), 1.27 (brs, 8H), $1.61(\mathrm{~m}, 2 \mathrm{H}), 3.67(\mathrm{~m}, 4 \mathrm{H}), 4.67(\mathrm{~m}$, $3 \mathrm{H}), 7.34(\mathrm{~m}, 10 \mathrm{H}) \mathrm{ppm}$.

${ }^{13} \mathrm{C}$ NMR $\left(125 \mathrm{MHz}, \mathrm{CDCl}_{3}\right) \delta 136.9,136.8,128.9,128.9,128.9,128.7,128.7,128.7,127.3$, $127.3,127.3,127.2,103.7,69.3,68.9,65.7,65.5,33.0,31.9,29.2,24.7,22.7,14.3$ ppm. 


\section{Entry 3}

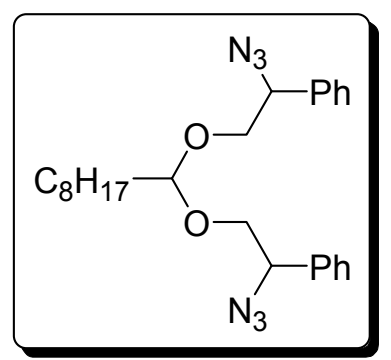

${ }^{1} \mathrm{H}$ NMR $\left(500 \mathrm{MHz}, \mathrm{CDCl}_{3}\right) \delta 0.88(\mathrm{~s}, 3 \mathrm{H}), 1.29$ (brs, 12H), 1.61 (brs, 2H), $3.67(\mathrm{~m}, 4 \mathrm{H}), 4.63(\mathrm{~m}$, $3 \mathrm{H}), 7.34(\mathrm{~m}, 10 \mathrm{H}) \mathrm{ppm}$.

${ }^{13} \mathrm{C}$ NMR $\left(125 \mathrm{MHz}, \mathrm{CDCl}_{3}\right) \delta 136.9,136.8,128.9,128.9,128.9,128.7,128.7,128.7,127.3$, $127.3,127.3,127.2,103.7,69.3,68.9,65.7,65.5,33.1,32.8,32.0,29.5,24.7,22.9,22.8,14.3$ ppm.

\section{Entry 4}

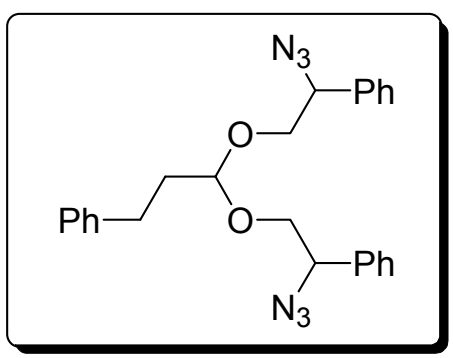

${ }^{1} \mathrm{H}$ NMR $\left(500 \mathrm{MHz}, \mathrm{CDCl}_{3}\right) \delta 1.99(\mathrm{~m}, 2 \mathrm{H}), 2.68(\mathrm{~m}, 2 \mathrm{H}), 3.69(\mathrm{~m}, 4 \mathrm{H}), 4.65(\mathrm{~m}, 3 \mathrm{H}), 7.28(\mathrm{~m}, 10$ H) $\mathrm{ppm}$.

${ }^{13} \mathrm{C}$ NMR $\left(125 \mathrm{MHz}, \mathrm{CDCl}_{3}\right) \delta 141.4,136.9,136.8,129.2,129.1,129.0,128.8,128.6,128.6$, 128.6, 128.6, 128.5, 127.3, 127.3, 127.3, 127.2, 126.5, 126.2, 102.9, 69.5, 69.1, 65.7, 65.5, 34.7, $31.0 \mathrm{ppm}$. 


\section{Entry 5}

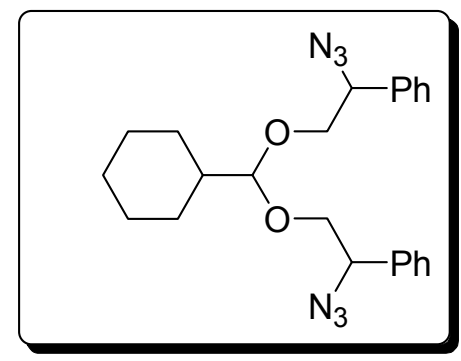

${ }^{1} \mathrm{H}$ NMR $\left(500 \mathrm{MHz}, \mathrm{CDCl}_{3}\right) \delta 1.13(\mathrm{~m}, 5 \mathrm{H}), 1.69(\mathrm{~m}, 6 \mathrm{H}), 3.68(\mathrm{~m}, 4 \mathrm{H}), 4.25(\mathrm{~m}, 1 \mathrm{H}), 4.67(\mathrm{~m}$, $2 \mathrm{H}), 7.35(\mathrm{~m}, 10 \mathrm{H}) \mathrm{ppm}$.

${ }^{13} \mathrm{C}$ NMR $\left(125 \mathrm{MHz}, \mathrm{CDCl}_{3}\right) \delta 136.9,136.9,129.0,128.9,128.8,128.7,128.7,127.4,127.4$, $127.3,127.3,127.2,107.4,69.9,69.7,65.8,65.6,40.3,28.2,28.1,26.5,25.9,25.8$ ppm.

Entry 6

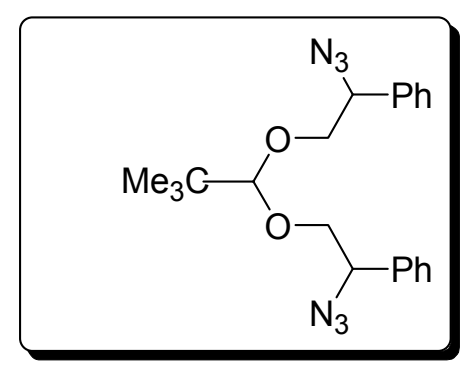

${ }^{1} \mathrm{H}$ NMR $\left(500 \mathrm{MHz}, \mathrm{CDCl}_{3}\right) \delta 0.92(\mathrm{~m}, 9 \mathrm{H}), 3.62(\mathrm{~m}, 2 \mathrm{H}), 3.83(\mathrm{~m}, 1 \mathrm{H}), 3.98(\mathrm{~m}, 1 \mathrm{H}), 4.07(\mathrm{~m}$, $1 \mathrm{H}), 4.71(\mathrm{~m}, 2 \mathrm{H}), 7.30(\mathrm{~m}, 10 \mathrm{H}) \mathrm{ppm}$.

${ }^{13} \mathrm{C}$ NMR $\left(500 \mathrm{MHz}, \mathrm{CDCl}_{3}\right) \delta 136.9,136.8,128.9,128.7,128.7,128.6,127.4,127.3,127.2$, $127.1,112.2,74.7,74.2,65.9,65.6,37.2,37.0,25.0,25.0 \mathrm{ppm}$. 


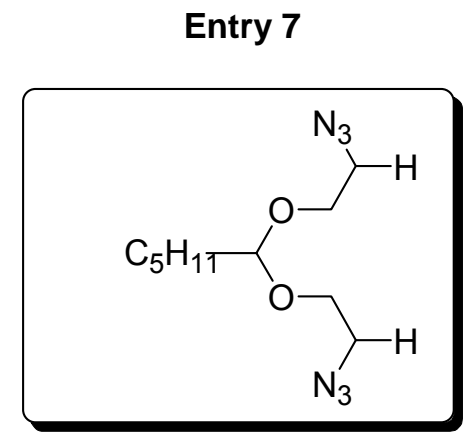

${ }^{1} \mathrm{H}$ NMR $\left(500 \mathrm{MHz}, \mathrm{CDCl}_{3}\right) \delta 0.87(\mathrm{brs}, 3 \mathrm{H}), 1.31(\mathrm{~m}, 6 \mathrm{H}), 1.63(\mathrm{~m}, 2 \mathrm{H}), 3.40(\mathrm{~m}, 4 \mathrm{H}), 3.62(\mathrm{~m}$, $2 \mathrm{H}), 3.76(\mathrm{~m}, 2 \mathrm{H}), 4.60(\mathrm{~m}, 1 \mathrm{H}) \mathrm{ppm}$.

${ }^{13} \mathrm{C}$ NMR $\left(125 \mathrm{MHz}, \mathrm{CDCl}_{3}\right) \delta$ 103.4, 63.9, 51.2, 32.9, 31.7, 24.4, 22.7, 14.2 ppm.

Entry 8

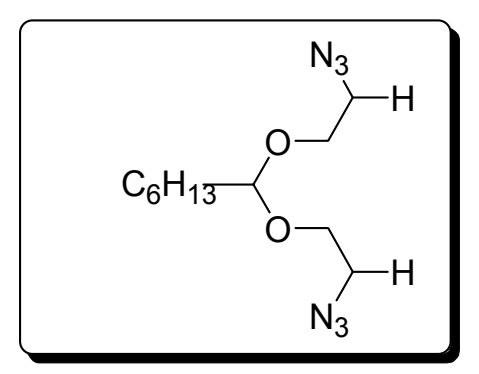

${ }^{1} \mathrm{H}$ NMR $\left(500 \mathrm{MHz}, \mathrm{CDCl}_{3}\right) \delta 0.88(\mathrm{~s}, 3 \mathrm{H}), 1.31(\mathrm{~m}, 8 \mathrm{H}), 1.64(\mathrm{~m}, 2 \mathrm{H}), 3.40(\mathrm{~m}, 4 \mathrm{H}), 3.62(\mathrm{~m}, 2 \mathrm{H})$, $3.76(\mathrm{~m}, 2 \mathrm{H}), 4.60(\mathrm{~m}, 1 \mathrm{H})$.

${ }^{13} \mathrm{C}$ NMR $\left(125 \mathrm{MHz}, \mathrm{CDCl}_{3}\right) \delta 103.4,63.9,51.2,33.0,31.9,29.2,24.7,22.7,14.2 \mathrm{ppm}$. 


\section{Entry 9}

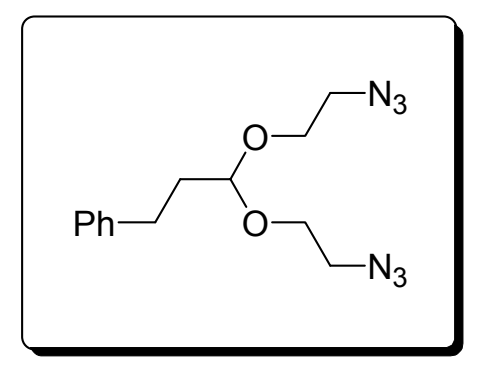

${ }^{1} \mathrm{H}$ NMR $\left(500 \mathrm{MHz}, \mathrm{CDCl}_{3}\right) \delta 2.0(\mathrm{~m}, 2 \mathrm{H}), 2.73(\mathrm{~m}, 2 \mathrm{H}), 3.41(\mathrm{~m}, 4 \mathrm{H}), 3.65(\mathrm{~m}, 2 \mathrm{H}), 3.79(\mathrm{~m}, 2 \mathrm{H})$, $4.62(\mathrm{t}, 1 \mathrm{H}), 7.26(\mathrm{~m}, 5 \mathrm{H}) \mathrm{ppm}$.

${ }^{13} \mathrm{C}$ NMR $\left(125 \mathrm{MHz}, \mathrm{CDCl}_{3}\right) \delta 141.4,128.7,128.6,128.6,128.6,126.2,102.6,64.2,51.2,34.6$, $31.0 \mathrm{ppm}$.

\section{Entry 10}

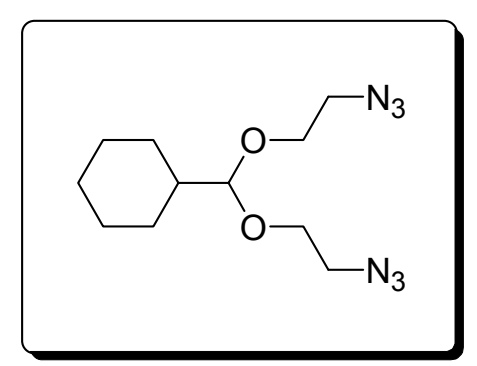

${ }^{1} \mathrm{H}$ NMR $\left(500 \mathrm{MHz}, \mathrm{CDCl}_{3}\right) \delta 1.12(\mathrm{~m}, 5 \mathrm{H}), 1.71(\mathrm{~m}, 6 \mathrm{H}), 3.40(\mathrm{~m}, 4 \mathrm{H}), 3.62(\mathrm{~m}, 2 \mathrm{H}), 3.75(\mathrm{~m}$, $2 \mathrm{H}), 4.23(\mathrm{~m}, 1 \mathrm{H}) \mathrm{ppm}$.

${ }^{13} \mathrm{C}$ NMR $\left(125 \mathrm{MHz}, \mathrm{CDCl}_{3}\right) \delta$ 107.1, 64.6, 51.2, 40.2, 28.1, 26.5, 26.1, 25.9, $25.2 \mathrm{ppm}$. 


\section{Characterization of Products in Table 2}

\section{Entry 1}

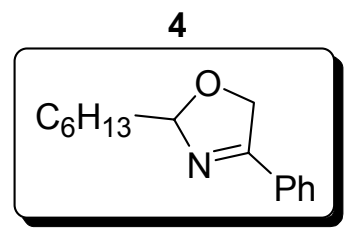

${ }^{1} \mathrm{H}$ NMR $\left(500 \mathrm{MHz}, \mathrm{CDCl}_{3}\right) \delta 0.89(\mathrm{~s}, 3 \mathrm{H}), 1.37(\mathrm{~m}, 6 \mathrm{H}), 1.80(\mathrm{~m}, 2 \mathrm{H}), 4.99(\mathrm{~m}, 4 \mathrm{H}), 5.89$ (brs, $1 \mathrm{H}), 7.48(\mathrm{~m}, 3 \mathrm{H}), 7.77(\mathrm{~m}, 2 \mathrm{H}) \mathrm{ppm}$.

${ }^{13} \mathrm{C}$ NMR $\left(125 \mathrm{MHz}, \mathrm{CDCl}_{3}\right) \delta 167.2,131.6,131.2,129.0,127.9,111.6,73.7,36.2,32.0,24.2$, 22.8, $14.3 \mathrm{ppm}$.

\section{Entry 2}

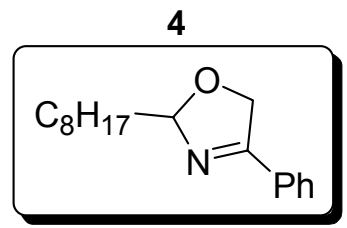

${ }^{1} \mathrm{H}$ NMR $\left(500 \mathrm{MHz}, \mathrm{CDCl}_{3}\right) \delta 0.88(\mathrm{~s}, 3 \mathrm{H}), 1.37(\mathrm{~m}, 6 \mathrm{H}), 1.75(\mathrm{~m}, 2 \mathrm{H}), 4.93(\mathrm{~m}, 1 \mathrm{H}), 5.05(\mathrm{~m}, 1 \mathrm{H})$, $5.89(\mathrm{~m}, 1 \mathrm{H}), 7.46(\mathrm{~m}, 3 \mathrm{H}), 7.75(\mathrm{~m}, 2 \mathrm{H}) \mathrm{ppm}$.

${ }^{13} \mathrm{C}$ NMR $\left(125 \mathrm{MHz}, \mathrm{CDCl}_{3}\right) \delta 167.2,131.6,131.2,128.9,127.9,107.6,73.7,36.2,32.1,29.8$, 29.7, 29.5, 24.5, 22.9, $14.3 \mathrm{ppm}$.

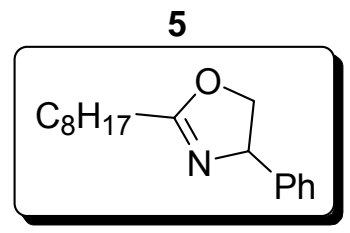

${ }^{1} \mathrm{H}$ NMR $\left(500 \mathrm{MHz}, \mathrm{CDCl}_{3}\right) \delta 0.88(\mathrm{brs}, 3 \mathrm{H}), 1.34(\mathrm{~m}, 10 \mathrm{H}), 1.71(\mathrm{~m}, 2 \mathrm{H}), 2.39(\mathrm{~m}, 2 \mathrm{H}), 4.07(\mathrm{~m}$, $1 \mathrm{H}), 4.60(\mathrm{~m}, 1 \mathrm{H}), 5.17(\mathrm{~m}, 1 \mathrm{H}), 7.30(\mathrm{~m}, 1 \mathrm{H}) \mathrm{ppm}$.

${ }^{13} \mathrm{C}$ NMR $\left(125 \mathrm{MHz}, \mathrm{CDCl}_{3}\right) \delta$ 169.2, 142.8, 128.9, 127.6, 126.8, 74.7, 69.8, 32.0, 29.5, 29.4, $29.4,28.3,26.3,22.9,14.3 \mathrm{ppm}$.

\section{Entry 3}

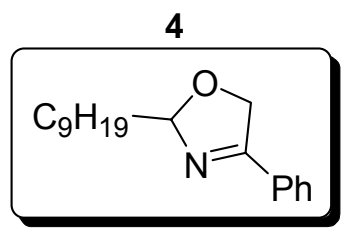


${ }^{1} \mathrm{H}$ NMR $\left(500 \mathrm{MHz}, \mathrm{CDCl}_{3}\right) \delta 0.87(\mathrm{~s}, 3 \mathrm{H}), 1.31(\mathrm{~m}, 12 \mathrm{H}), 1.46(\mathrm{~m}, 2 \mathrm{H}), 1.80(\mathrm{~m}, 2 \mathrm{H}), 4.92(\mathrm{dd}, \mathrm{J}$ $=3.5,13.5 \mathrm{~Hz}, 1 \mathrm{H}), 5.03(\mathrm{dd}, \mathrm{J}=6.0,13.9 \mathrm{~Hz}, 1 \mathrm{H}), 5.87(\mathrm{~m}, 1 \mathrm{H}), 7.45(\mathrm{~m}, 3 \mathrm{H}), 7.74(\mathrm{~m}, 2 \mathrm{H})$ ppm.

${ }^{13} \mathrm{C}$ NMR $\left(125 \mathrm{MHz}, \mathrm{CDCl}_{3}\right) \delta 167.2,131.6,131.3,129.0,127.9,107.6,73.7,36.3,32.1,29.8$, 29.8, 29.8, 29.5, 24.6, 22.9, $14.4 \mathrm{ppm}$.

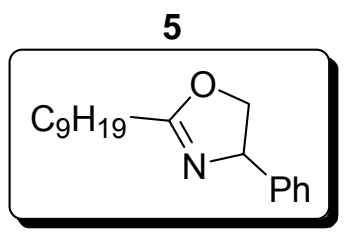

${ }^{1} \mathrm{H}$ NMR $\left(500 \mathrm{MHz}, \mathrm{CDCl}_{3}\right) \delta 0.87(\mathrm{~s}, 3 \mathrm{H}), 1.33(\mathrm{~m}, 12 \mathrm{H}), 1.69(\mathrm{~m}, 2 \mathrm{H}), 2.38(\mathrm{~m}, 2 \mathrm{H}), 4.05(\mathrm{~m}$, $1 \mathrm{H}), 4.57(\mathrm{~m}, 1 \mathrm{H}), 5.15(\mathrm{~m}, 1 \mathrm{H}), 7.29(\mathrm{~m}, 5 \mathrm{H}) \mathrm{ppm}$.

${ }^{13} \mathrm{C}$ NMR $\left(125 \mathrm{MHz}, \mathrm{CDCl}_{3}\right) \delta 169.0,142.6,128.6,128.6,127.4,126.5,74.4,69.5,31.8,29.4$, 29.2, 29.2, 28.0, 26.1, 22.6, $14.0 \mathrm{ppm}$.

\section{Entry 4}

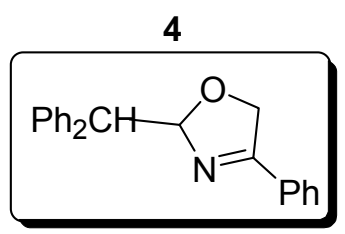

${ }^{1} \mathrm{H}$ NMR $\left(500 \mathrm{MHz}, \mathrm{CDCl}_{3}\right) \delta 4.30(\mathrm{dd}, \mathrm{J}=6.0,13.5 \mathrm{~Hz}, 1 \mathrm{H}), 4.45(\mathrm{~d}, \mathrm{~J}=3 \mathrm{~Hz}, 1 \mathrm{H}), 4.77(\mathrm{dd}, \mathrm{J}=$ 3.5, $13.5 \mathrm{~Hz}, 1 \mathrm{H}), 6.53(\mathrm{~m}, 1 \mathrm{H}), 7.15(\mathrm{~m}, 8 \mathrm{H}), 7.35(\mathrm{~m}, 5 \mathrm{H}), 7.55(\mathrm{~m}, 2 \mathrm{H}) \mathrm{ppm}$.

${ }^{13} \mathrm{C}$ NMR $\left(125 \mathrm{MHz}, \mathrm{CDCl}_{3}\right) \delta 168.2,140.9,139.9,131.3,130.8,129.7,129.4,128.6,128.0$, $127.9,127.6,126.4,126.4,108.6,74.0,56.5 \mathrm{ppm}$.

\section{Entry 5}

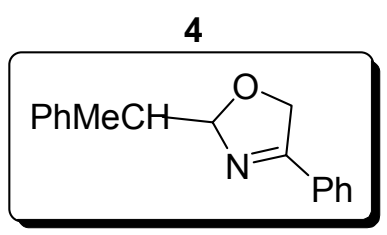

${ }^{1} \mathrm{H}$ NMR $\left(500 \mathrm{MHz}, \mathrm{CDCl}_{3}\right) \delta 1.41(\mathrm{dd}, \mathrm{J}=7.0,42.0 \mathrm{~Hz}, 3 \mathrm{H}), 3.30(\mathrm{~d}, \mathrm{~J}=42.0 \mathrm{~Hz}, 1 \mathrm{H}), 4.75$ (dd, $\mathrm{J}=6.0,13.0 \mathrm{~Hz}, 1 \mathrm{H}), 4.91(\mathrm{~m}, 1 \mathrm{H}), 6.07(\mathrm{~m}, 1 \mathrm{H}), 7.36(\mathrm{~m}, 8 \mathrm{H}), 7.72(\mathrm{~m}, 2 \mathrm{H}) \mathrm{ppm}$.

${ }^{13} \mathrm{C}$ NMR $\left(125 \mathrm{MHz}, \mathrm{CDCl}_{3}\right) \delta 168.3,142.5,142.2,131.6,131.0,128.9,128.9,128.7,128.7$, $128.2,127.9,126.7,126.7,110.8,74.5,47.2,14.6 \mathrm{ppm}$.

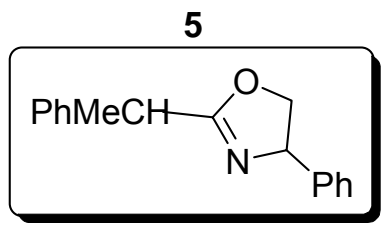

${ }^{1} \mathrm{H}$ NMR $\left(500 \mathrm{MHz}, \mathrm{CDCl}_{3}\right) \delta 1.66(\mathrm{~m}, 3 \mathrm{H}), 3.89(\mathrm{~m}, 1 \mathrm{H}), 4.07(\mathrm{dd}, \mathrm{J}=8.0,16.0 \mathrm{~Hz}, 1 \mathrm{H}), 4.60(\mathrm{~m}$, $1 \mathrm{H}), 5.21(\mathrm{~m}, 1 \mathrm{H}), 7.32(\mathrm{~m}, 10 \mathrm{H}) \mathrm{ppm}$.

${ }^{13} \mathrm{C}$ NMR $\left(125 \mathrm{MHz}, \mathrm{CDCl}_{3}\right) \delta 171.0,142.7,141.6,128.9,128.9,127.8,127.7,127.7,127.6$. $127.4,127.3,126.9,126.8,75.1,69.7,39.8,19.7 \mathrm{ppm}$. 


\section{Entry 6}

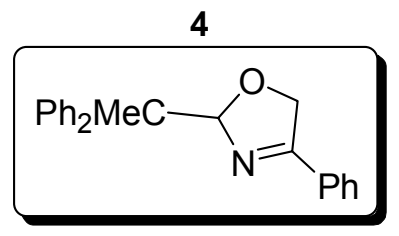

${ }^{1} \mathrm{H}$ NMR $\left(500 \mathrm{MHz}, \mathrm{CDCl}_{3}\right) \delta 1.82(\mathrm{~s}, 3 \mathrm{H}), 4.36(\mathrm{dd}, \mathrm{J}=6.0,13.5 \mathrm{~Hz}, 1 \mathrm{H}), 4.84(\mathrm{dd}, \mathrm{J}=3.0,13.0$ $\mathrm{Hz}, 1 \mathrm{H}), 6.71$ (brs, 1H), $7.41(\mathrm{~m}, 15 \mathrm{H}) \mathrm{ppm}$.

${ }^{13} \mathrm{C}$ NMR $\left(125 \mathrm{MHz}, \mathrm{CDCl}_{3}\right) \delta 168.6,146.9,145.7,131.5,131.0,129.3,129.2,129.1,128.8$, $128.8,128.7,128.7,127.9,127.9,127.6,127.3,126.2,126.2,111.4,74.5,53.4,25.0$ ppm.

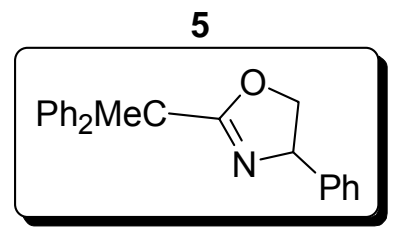

${ }^{1} \mathrm{H}$ NMR $\left(500 \mathrm{MHz}, \mathrm{CDCl}_{3}\right) \delta 2.07(\mathrm{brs}, 3 \mathrm{H}), 4.14(\mathrm{~m}, 1 \mathrm{H}), 4.67(\mathrm{~m}, 1 \mathrm{H}), 5.27(\mathrm{~m}, 1 \mathrm{H}), 7.33(\mathrm{~m}$, $15 \mathrm{H}) \mathrm{ppm}$.

${ }^{13} \mathrm{C}$ NMR $\left(125 \mathrm{MHz}, \mathrm{CDCl}_{3}\right) \delta 172.2,145.0,145.0,142.6,128.9,128.3,128.3,128.2,128.2$, $127.7,127.0,127.0,126.8,75.4,69.7,50.8,28.7 \mathrm{ppm}$.

\section{Entry 7}

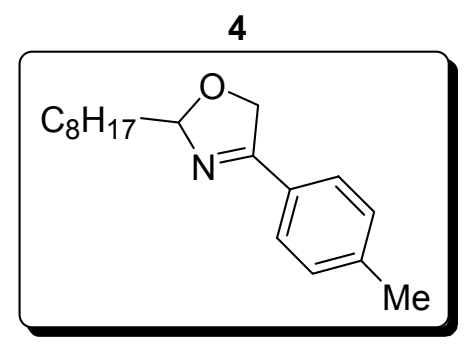

${ }^{1} \mathrm{H}$ NMR $\left(500 \mathrm{MHz}, \mathrm{CDCl}_{3}\right) \delta 0.89(\mathrm{t}, \mathrm{J}=6.0 \mathrm{~Hz}, 3 \mathrm{H}), 1.38(\mathrm{~m}, 12 \mathrm{H}), 1.81(\mathrm{~m}, 2 \mathrm{H}), 2.42(\mathrm{~s}, 3 \mathrm{H})$, $4.93(\mathrm{dd}, \mathrm{J}=4.0,13.5 \mathrm{~Hz}, 1 \mathrm{H}), 5.05(\mathrm{dd}, \mathrm{J}=5.5,13.5 \mathrm{~Hz}, 1 \mathrm{H}), 5.89(\mathrm{t}, J=4.5 \mathrm{~Hz}, 1 \mathrm{H}), 7.27(\mathrm{~m}$, $3 \mathrm{H}), 7.66(\mathrm{~m}, 2 \mathrm{H}) \mathrm{ppm}$.

${ }^{13} \mathrm{C}$ NMR $\left(125 \mathrm{MHz}, \mathrm{CDCl}_{3}\right) \delta 167.1,142.0,129.7,129.6,128.5,127.9,127.9,107.5,73.7,36.3$, $32.1,29.9,29.5,24.5,22.9,21.8,21.8,14.3 \mathrm{ppm}$.

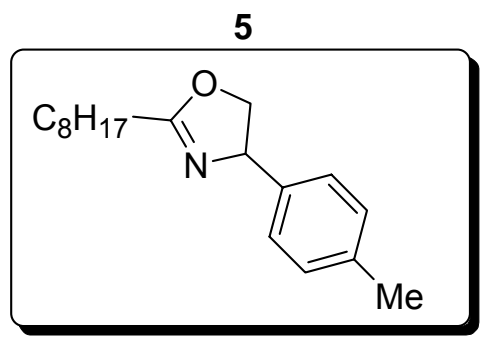


${ }^{1} \mathrm{H}$ NMR (500 MHz, CDCl $\left.)_{3}\right) \delta 0.90(\mathrm{t}, \mathrm{J}=7.0 \mathrm{~Hz}, 3 \mathrm{H}), 1.35(\mathrm{~m}, 12 \mathrm{H}), 1.71(\mathrm{~m}, 2 \mathrm{H}), 2.35(\mathrm{~s}, 3 \mathrm{H})$, $4.07(\mathrm{t}, J=8.0 \mathrm{~Hz}, 1 \mathrm{H}), 4.59$ (dd, $J=8.5,10.5 \mathrm{~Hz}, 1 \mathrm{H}), 5.15(\mathrm{t}, J=9.0 \mathrm{~Hz}, 1 \mathrm{H}), 7.17(\mathrm{~m}, 4 \mathrm{H}) \mathrm{ppm}$. ${ }^{13} \mathrm{C}$ NMR $\left(125 \mathrm{MHz}, \mathrm{CDCl}_{3}\right) \delta 169.3,139.8,137.3,129.5,126.7,74.8,69.4,37.0,32.0,29.5$, 29.4, 28.3, 26.3, 22.9, 21.3, $14.3 \mathrm{ppm}$.

\section{Entry 8}

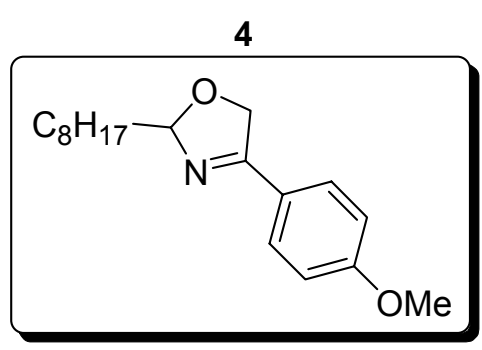

${ }^{1} \mathrm{H}$ NMR $\left(500 \mathrm{MHz}, \mathrm{CDCl}_{3}\right) \delta 0.90(\mathrm{t}, \mathrm{J}=6.5 \mathrm{~Hz}, 3 \mathrm{H}), 1.33(\mathrm{~m}, 10 \mathrm{H}), 1.47(\mathrm{~m}, 2 \mathrm{H}), 1.80(\mathrm{~m}, 2 \mathrm{H})$, $3.88(\mathrm{~s}, 3 \mathrm{H}), 4.91(\mathrm{dd}, \mathrm{J}=4.0,13.0 \mathrm{~Hz}, 1 \mathrm{H}), 5.03(\mathrm{dd}, \mathrm{J}=5.5,13.0 \mathrm{~Hz}, 1 \mathrm{H}), 5.87(\mathrm{~m}, 1 \mathrm{H}), 6.97$ $(\mathrm{d}, J=9.0 \mathrm{~Hz}, 2 \mathrm{H}), 7.71(\mathrm{~d}, J=8.5 \mathrm{~Hz}, 2 \mathrm{H}) \mathrm{ppm}$.

13C NMR $\left(125 \mathrm{MHz}, \mathrm{CDCl}_{3}\right) \delta 166.5,129.6,124.0,114.3,107.5,73.6,36.4,32.1,29.8,29.8$, 29.5, 24.6, 22.9, $14.4 \mathrm{ppm}$.

\section{Entry 9}

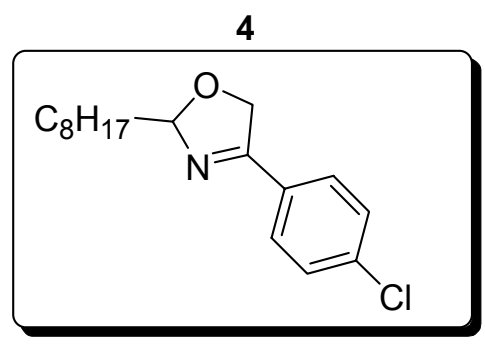

${ }^{1} \mathrm{H}$ NMR $\left(500 \mathrm{MHz}, \mathrm{CDCl}_{3}\right) \delta 0.90$ (brs, 3H), $1.40(\mathrm{~m}, 12 \mathrm{H}), 1.82(\mathrm{~m}, 2 \mathrm{H}), 4.94(\mathrm{~m}, 1 \mathrm{H}), 5.04(\mathrm{~m}$, $1 \mathrm{H}), 5.90(\mathrm{~m}, 1 \mathrm{H}), 7.45(\mathrm{~m}, 2 \mathrm{H}), 7.72(\mathrm{~m}, 2 \mathrm{H}) \mathrm{ppm}$.

${ }^{13} \mathrm{C}$ NMR $\left(125 \mathrm{MHz}, \mathrm{CDCl}_{3}\right) \delta 166.5,129.6,124.0,114.3,107.3,73.6,36.4,32.1,29.8,29.8$, 29.5, 24.6, 22.9, $14.4 \mathrm{ppm}$.

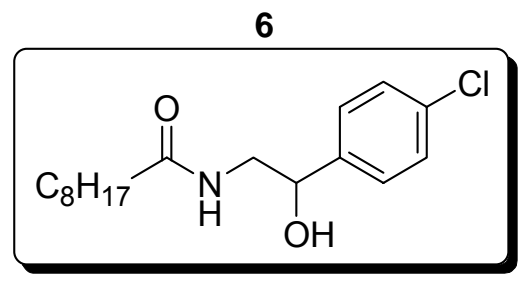

${ }^{1} \mathrm{H}$ NMR (500 MHz, CDCl $)$ ) 0.77 (brs, 3H), $1.15(\mathrm{~m}, 10 \mathrm{H}), 1.52(\mathrm{~m}, 2 \mathrm{H}), 2.14(\mathrm{~m}, 2 \mathrm{H}), 3.75(\mathrm{~m}$, $2 \mathrm{H}), 4.93(\mathrm{~m}, 1 \mathrm{H}), 6.11(\mathrm{~m}, 1 \mathrm{H}), 7.18(\mathrm{~m}, 4 \mathrm{H}) \mathrm{ppm}$.

${ }^{13} \mathrm{C}$ NMR $\left(125 \mathrm{MHz}, \mathrm{CDCl}_{3}\right) \delta 174.0,137.9,133.8,129.2,128.3,66.4,55.2,36.9,32.0,29.5$, $29.4,29.4,25.9,22.9,14.3 \mathrm{ppm}$. 


\section{Entry 10}

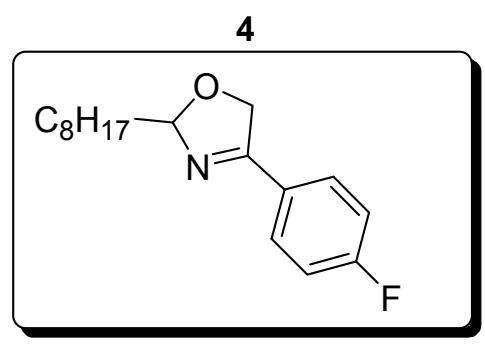

${ }^{1} \mathrm{H}$ NMR $\left(500 \mathrm{MHz}, \mathrm{CDCl}_{3}\right) \delta 0.89(\mathrm{~m}, 3 \mathrm{H}), 1.40(\mathrm{~m}, 12 \mathrm{H}), 1.75(\mathrm{~m}, 2 \mathrm{H}), 4.92(\mathrm{dd}, \mathrm{J}=4.0,13.5$ $\mathrm{Hz}, 1 \mathrm{H}), 5.04(\mathrm{dd}, \mathrm{J}=6.0,13.5 \mathrm{~Hz}, 1 \mathrm{H}), 5.88(\mathrm{t}, \mathrm{J}=5.0 \mathrm{~Hz}, 1 \mathrm{H}), 7.15(\mathrm{~m}, 2 \mathrm{H}), 7.77(\mathrm{~m}, 2 \mathrm{H}) \mathrm{ppm}$. ${ }^{13} \mathrm{C}$ NMR $\left(125 \mathrm{MHz}, \mathrm{CDCl}_{3}\right) \delta 166.0,130.1,130.0,116.2,116.0,107.6,73.6,33.2,32.1,29.9$, 29.8, 29.5, 24.6, 22.9, $14.3 \mathrm{ppm}$.

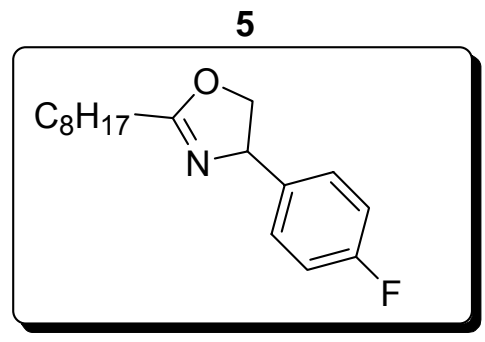

${ }^{1} \mathrm{H}$ NMR $\left(500 \mathrm{MHz}, \mathrm{CDCl}_{3}\right) \delta 0.90(\mathrm{t}, \mathrm{J}=6.5 \mathrm{~Hz}, 3 \mathrm{H}), 1.36(\mathrm{~m}, 10 \mathrm{H}), 1.73(\mathrm{~m}, 2 \mathrm{H}), 2.41(\mathrm{~m}, 2 \mathrm{H})$, $4.04(\mathrm{~m}, 1 \mathrm{H}), 4.60(\mathrm{~m}, 1 \mathrm{H}), 5.17(\mathrm{~m}, 1 \mathrm{H}), 7.02(\mathrm{~m}, 2 \mathrm{H}), 7.25(\mathrm{~m}, 2 \mathrm{H}) \mathrm{ppm}$.

${ }^{13} \mathrm{C}$ NMR $\left(125 \mathrm{MHz}, \mathrm{CDCl}_{3}\right) \delta 173.9,138.6,135.2,128.4,128.4,74.8,69.1,32.0,29.9,29.4$, 28.3, 26.3, 25.9, 22.8, $14.3 \mathrm{ppm}$.

6

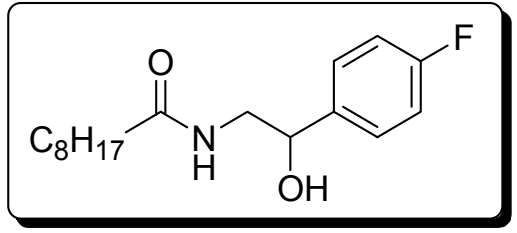

${ }^{1} \mathrm{H}$ NMR (500 MHz, CDCl 3 ) $\delta 0.90(\mathrm{t}, \mathrm{J}=5 \mathrm{~Hz}, 3 \mathrm{H}), 1.28(\mathrm{~m}, 11 \mathrm{H}), 1.66(\mathrm{~m}, 2 \mathrm{H}), 2.27(\mathrm{t}, \mathrm{J}=7.5$ $\mathrm{Hz}, 2 \mathrm{H}), 3.90(\mathrm{~d}, \mathrm{~J}=5.0 \mathrm{~Hz}, 2 \mathrm{H}), 5.07(\mathrm{~m}, 1 \mathrm{H}), 6.16(\mathrm{~m}, 1 \mathrm{H}), 7.07(\mathrm{~m}, 2 \mathrm{H}), 7.30(\mathrm{~m}, 2 \mathrm{H}) \mathrm{ppm}$.

${ }^{13} \mathrm{C}$ NMR $\left(125 \mathrm{MHz}, \mathrm{CDCl}_{3}\right) \delta 174.0,135.1,128.6,116.1,115.9,66.7,55.4,37.0,32.0,29.9$, 29.5, 29.4, 25.9, 22.9, $14.3 \mathrm{ppm}$. 


\section{Entry 11}

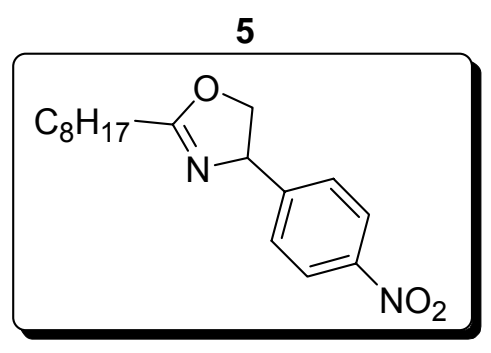

${ }^{1} \mathrm{H}$ NMR $\left(500 \mathrm{MHz}, \mathrm{CDCl}_{3}\right) \delta 0.90(\mathrm{t}, \mathrm{J}=6.5 \mathrm{~Hz}, 3 \mathrm{H}), 1.35(\mathrm{~m}, 10 \mathrm{H}), 1.73(\mathrm{~m}, 2 \mathrm{H}), 2.42(\mathrm{~m}, 2 \mathrm{H})$, $3.74(\mathrm{dd}, J=7.5,14.0 \mathrm{~Hz}, 1 \mathrm{H}), 4.35(\mathrm{dd}, J=10.0,14.0 \mathrm{~Hz}, 1 \mathrm{H}), 5.56(\mathrm{dd}, \mathrm{J}=7.5,10.0 \mathrm{~Hz}, 1 \mathrm{H})$, $7.46(\mathrm{~d}, \mathrm{~J}=8.5 \mathrm{~Hz}, 2 \mathrm{H}), 8.26(\mathrm{~d}, \mathrm{~J}=8.0 \mathrm{~Hz}, 2 \mathrm{H}) \mathrm{ppm}$.

${ }^{13} \mathrm{C}$ NMR $(125 \mathrm{MHz}, \mathrm{CDCl} 3) \delta 168.3,148.8,147.9,126.4,124.3,79.4,63.0,32.0,29.5,29.5$, $29.4,28.3,26.2,22.9,14.3 \mathrm{ppm}$.

6

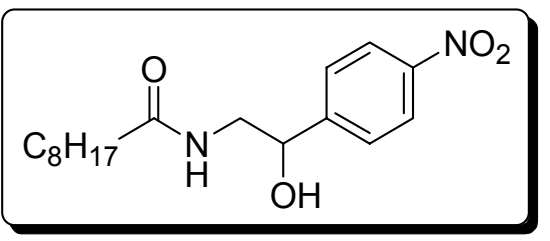

${ }^{1} \mathrm{H}$ NMR $\left(500 \mathrm{MHz}, \mathrm{CDCl}_{3}\right) \delta 0.90(\mathrm{t}, \mathrm{J}=6.0 \mathrm{~Hz}, 3 \mathrm{H}), 1.30(\mathrm{~m}, 11 \mathrm{H}), 1.62(\mathrm{~m}, 2 \mathrm{H}), 2.21(\mathrm{~m}, 2 \mathrm{H})$, $3.40(\mathrm{~m}, 1 \mathrm{H}), 3.75(\mathrm{~m}, 1 \mathrm{H}), 5.02(\mathrm{dd}, \mathrm{J}=2.5,7.0 \mathrm{~Hz}, 1 \mathrm{H}), 5.96(\mathrm{brs}, 1 \mathrm{H}), 7.57(\mathrm{~d}, J=9.0 \mathrm{~Hz}, 2 \mathrm{H})$, $8.22(\mathrm{~d}, \mathrm{~J}=8.0 \mathrm{~Hz}, 2 \mathrm{H}) \mathrm{ppm}$.

${ }^{13} \mathrm{C}$ NMR $\left(125 \mathrm{MHz}, \mathrm{CDCl}_{3}\right) \delta 176.0,149.6,147.6,126.9,123.9,73.8,48.1,36.6,32.0,29.5,29.4$ ,29.3, 25.8, 22.9, $14.3 \mathrm{ppm}$. 


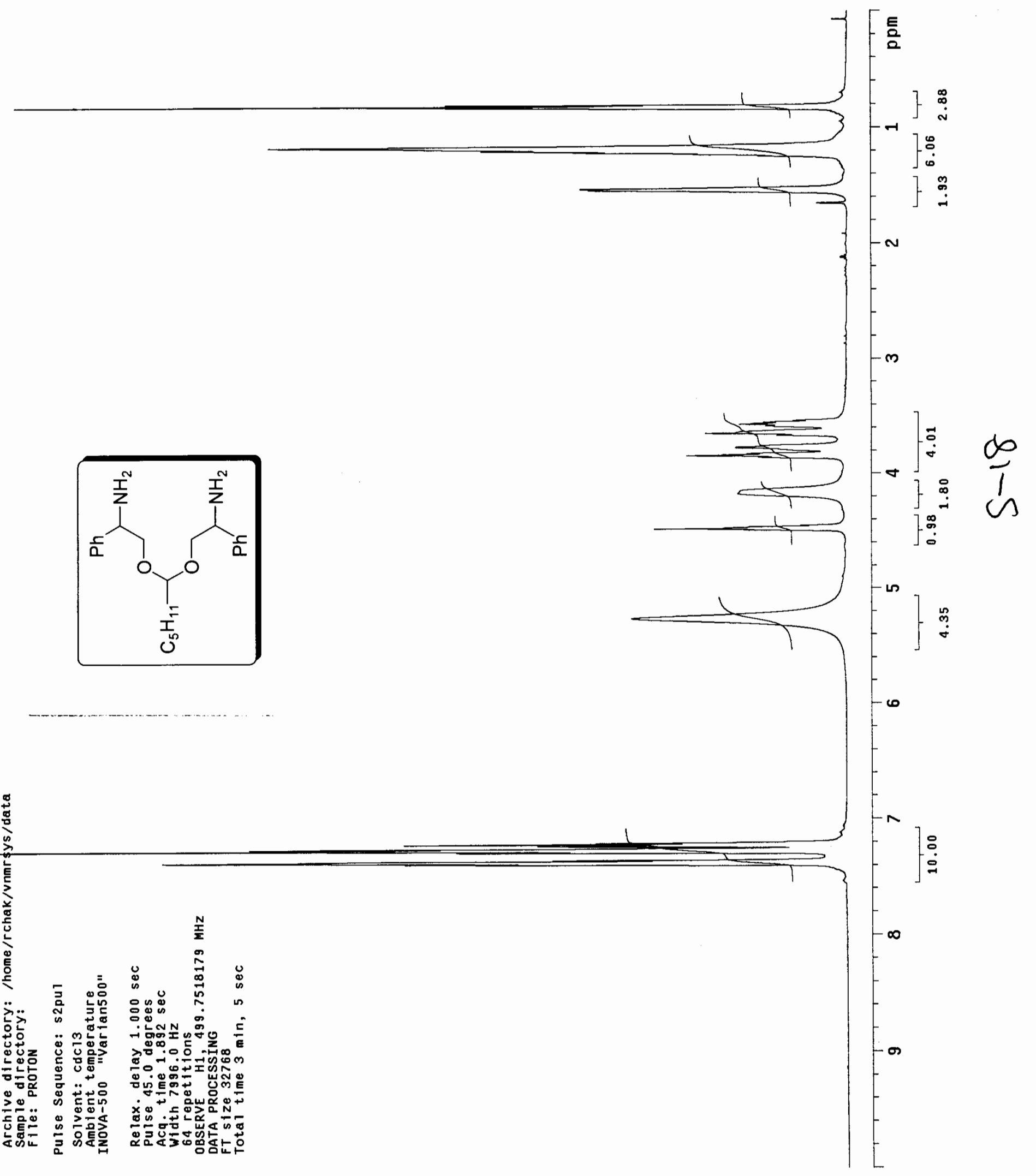




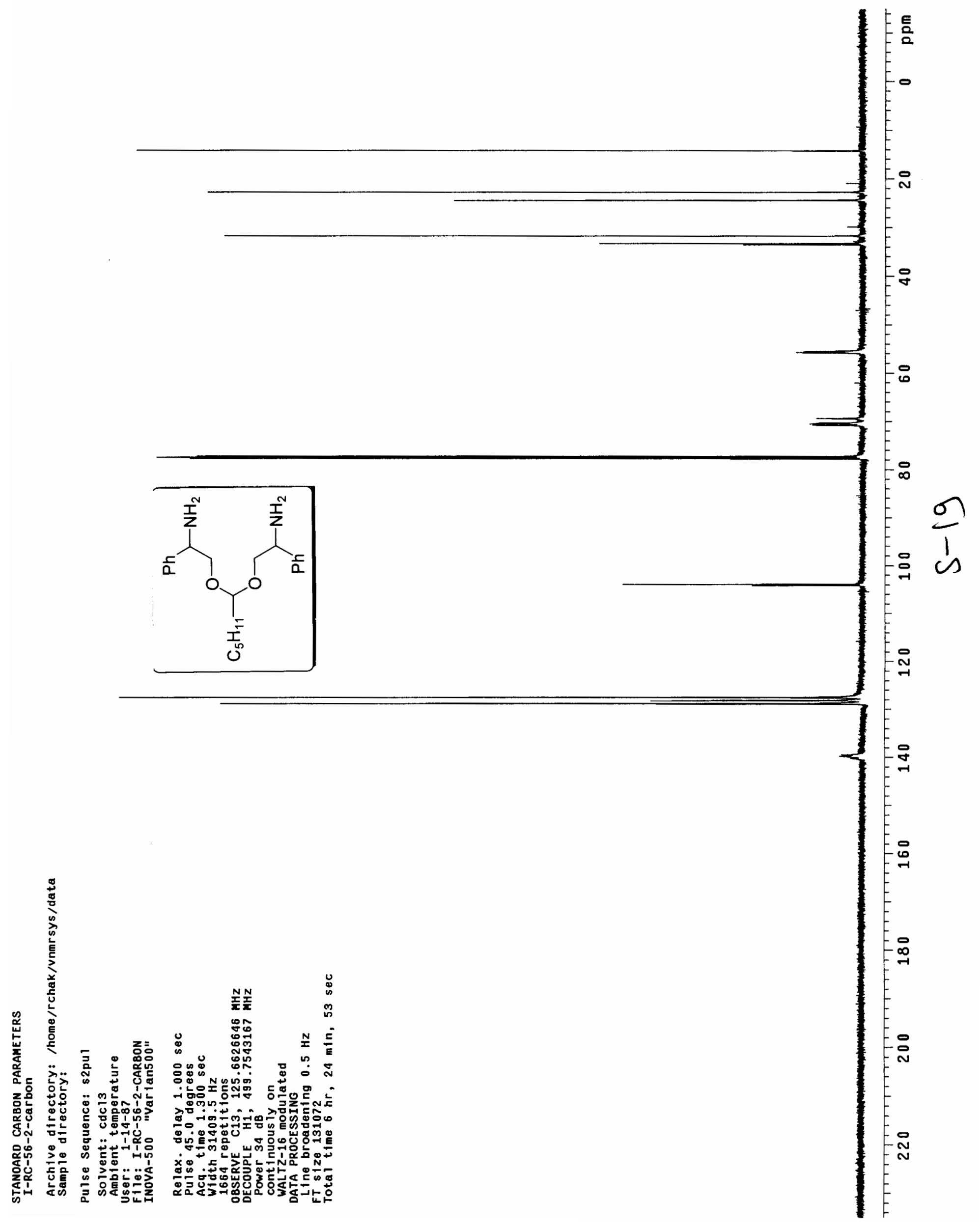




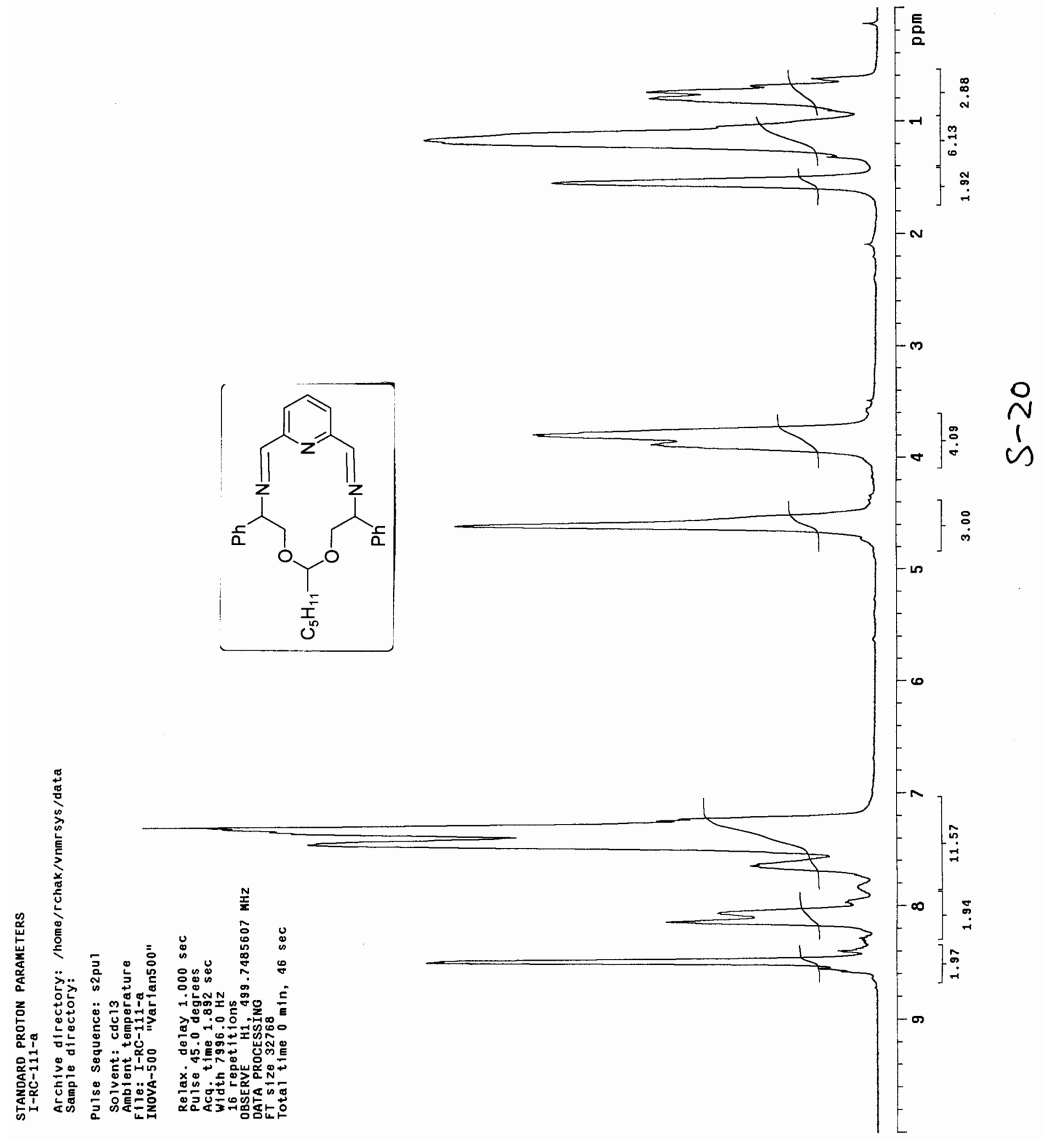




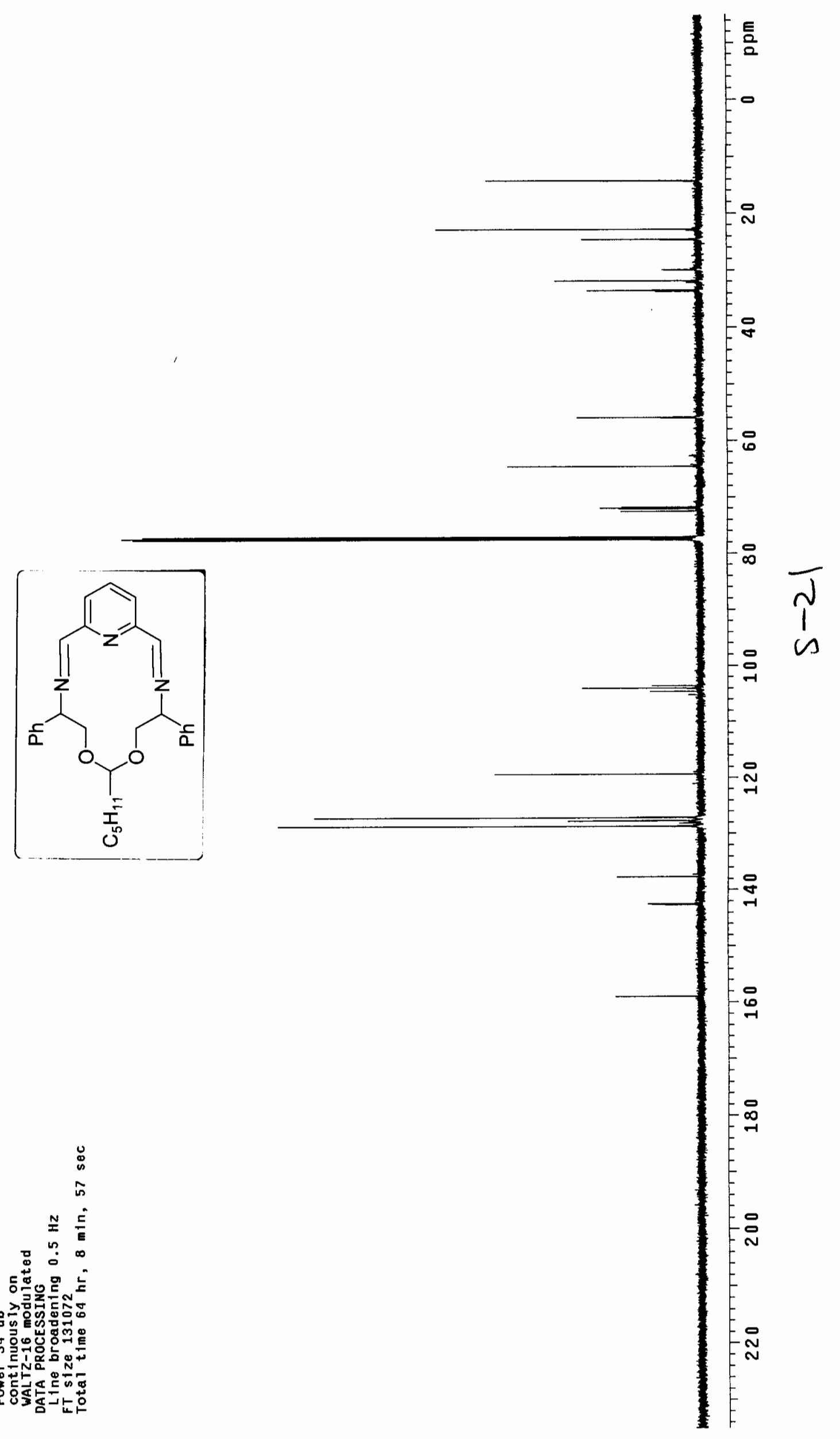




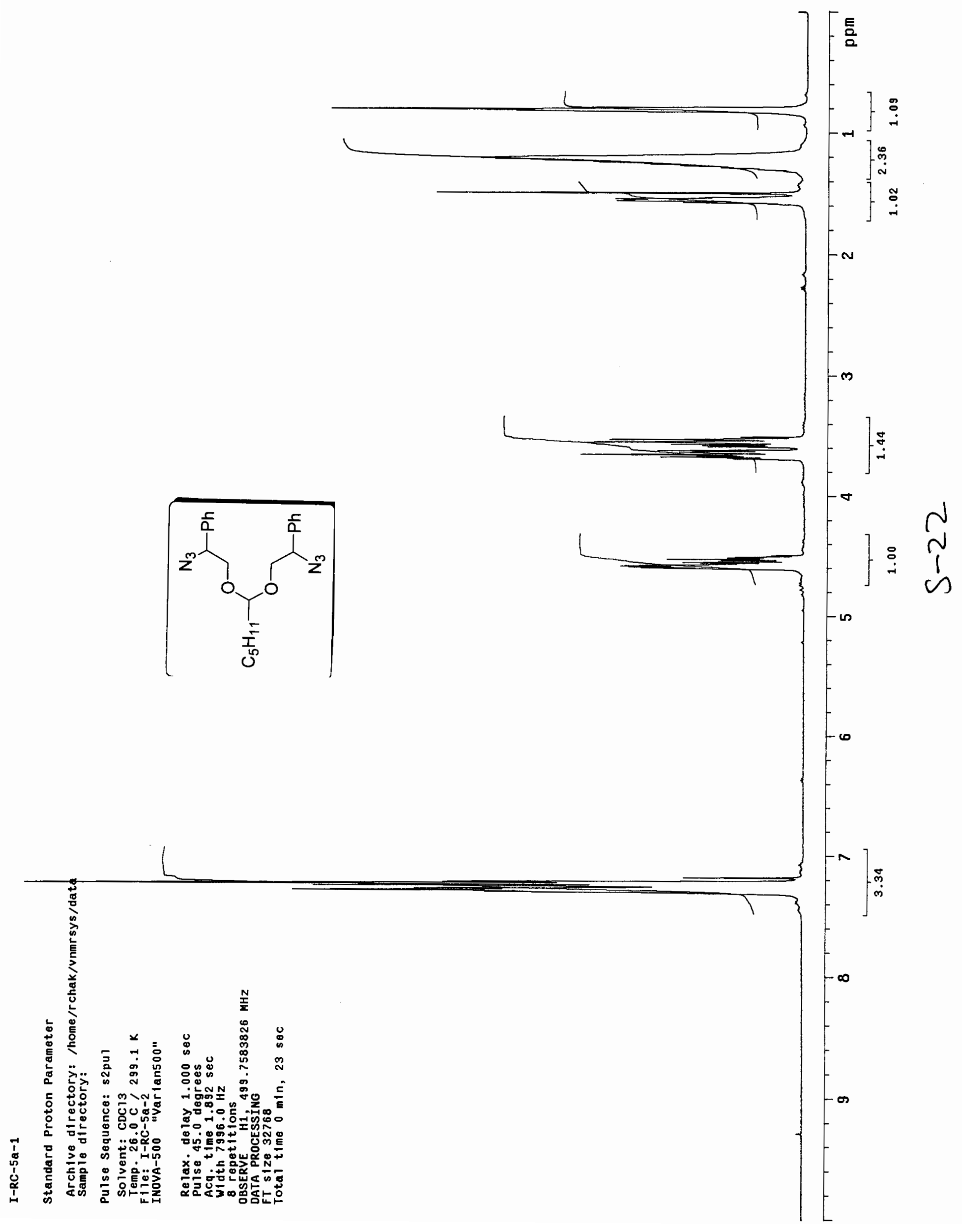



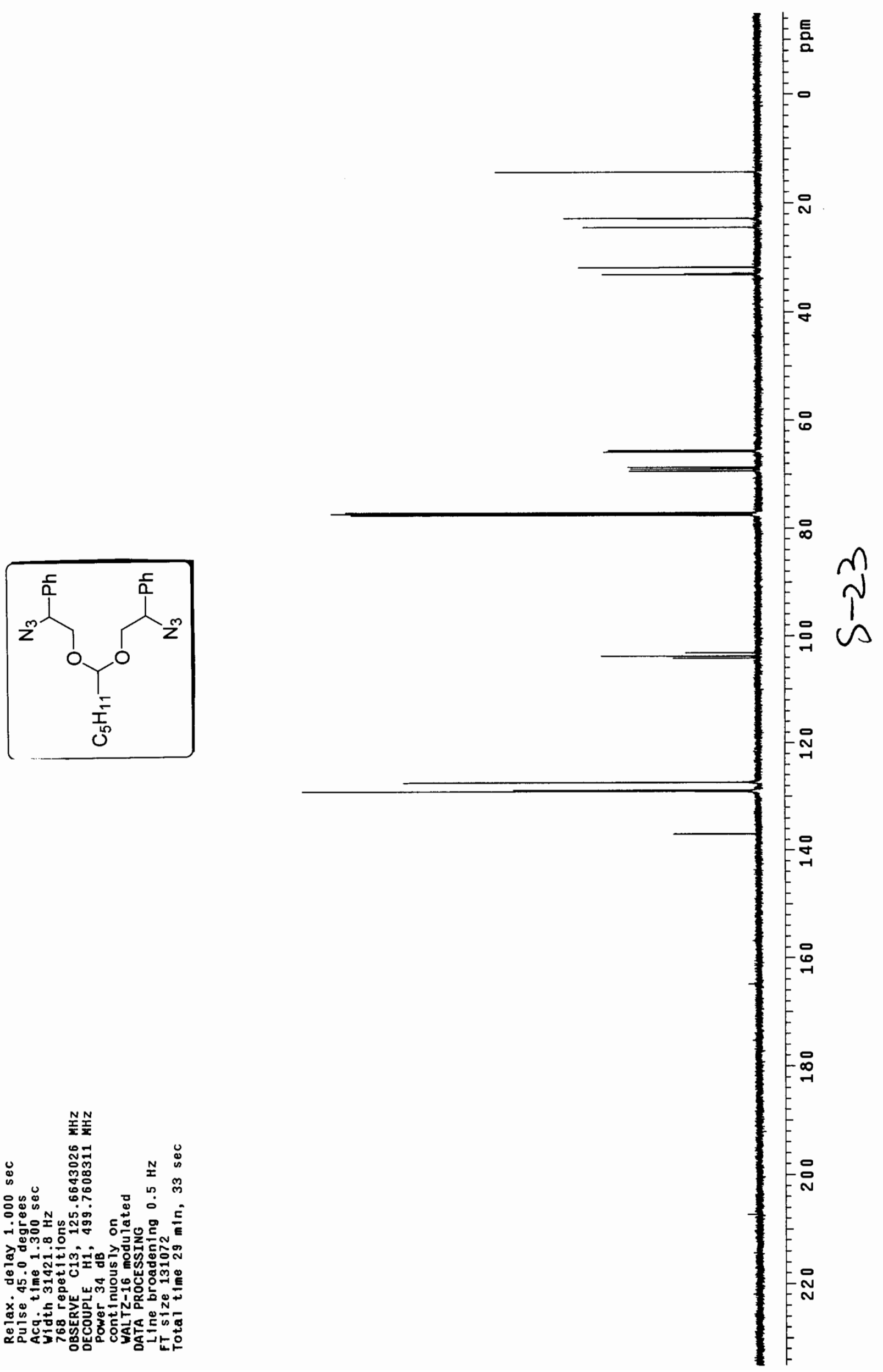


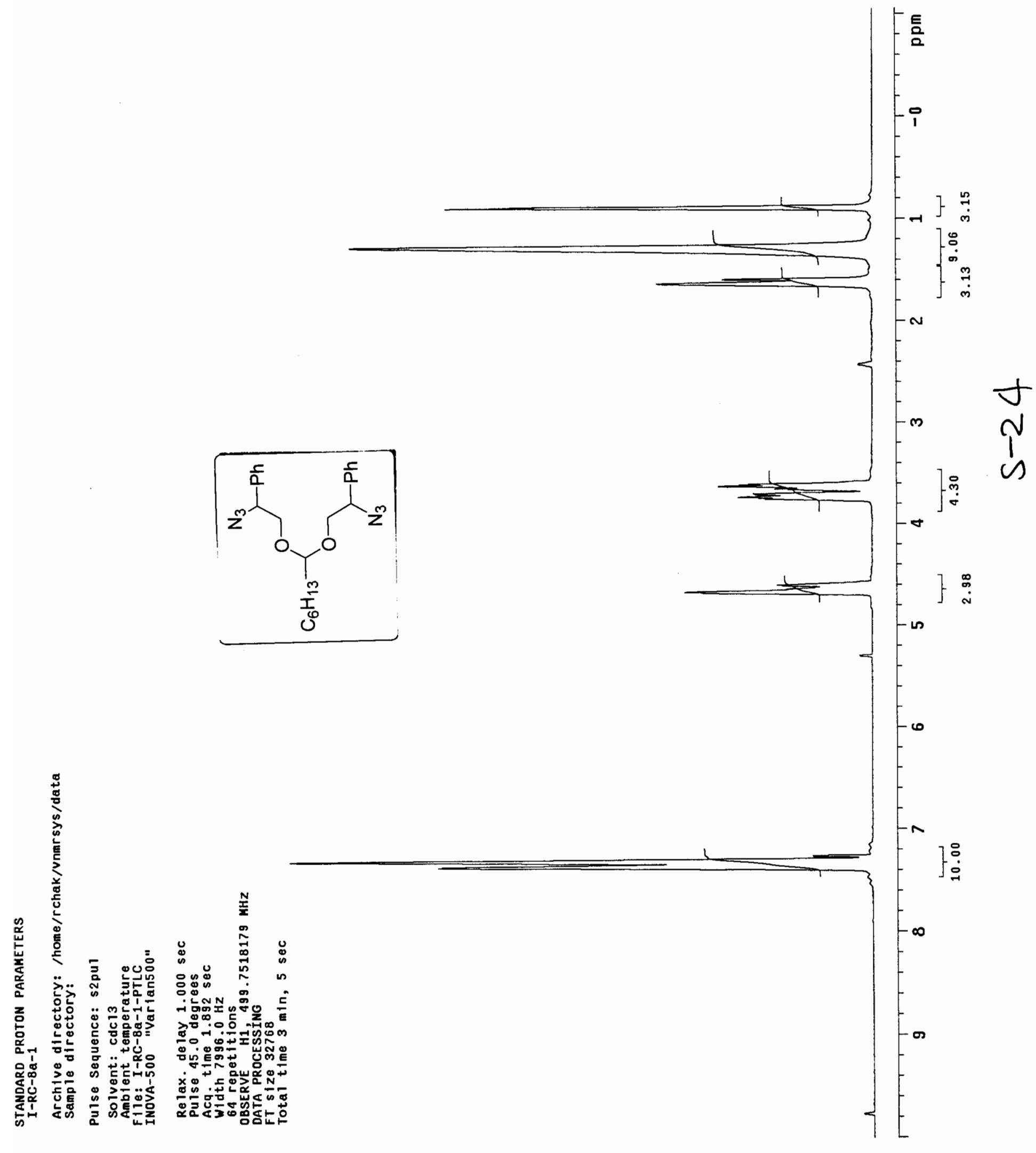




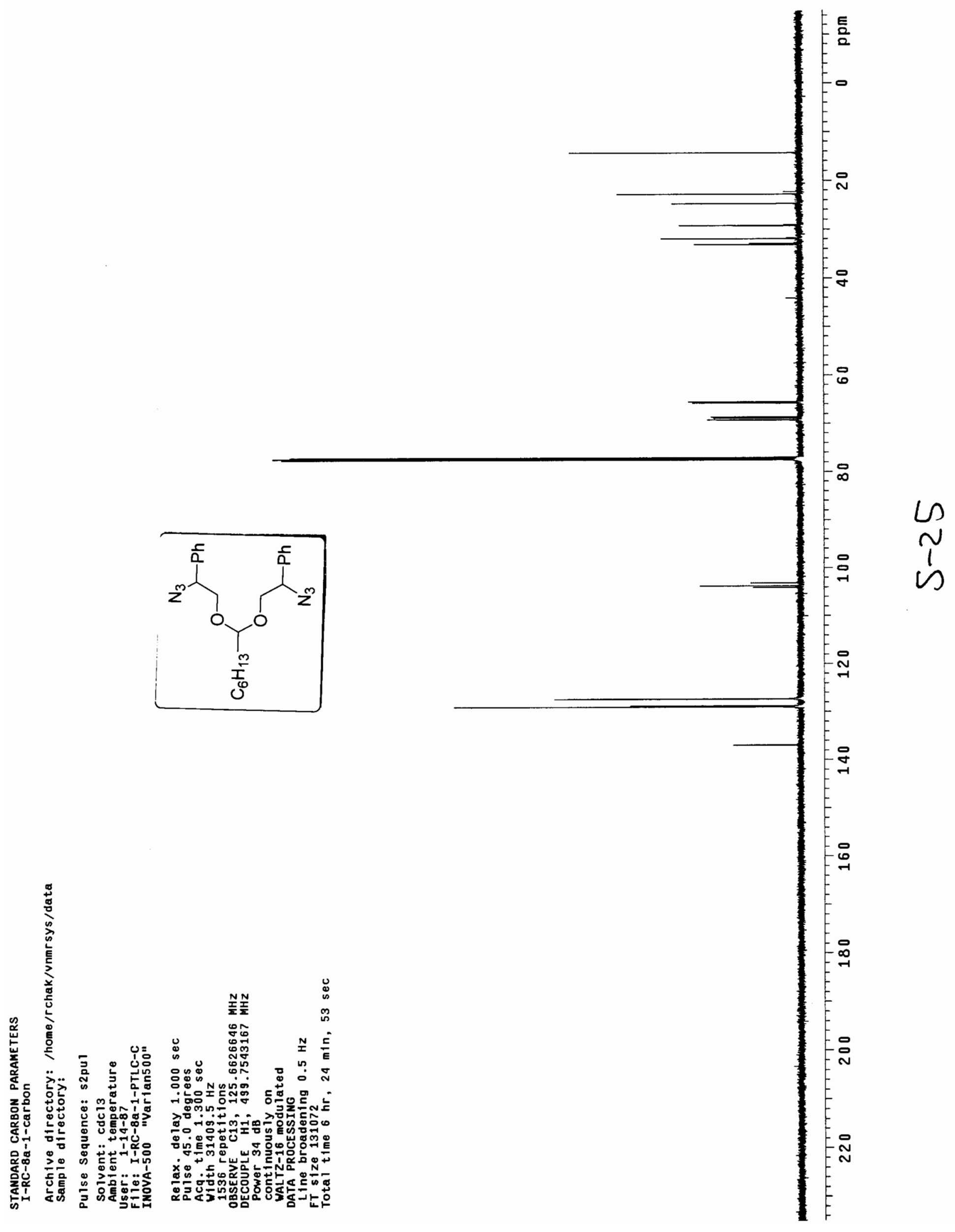




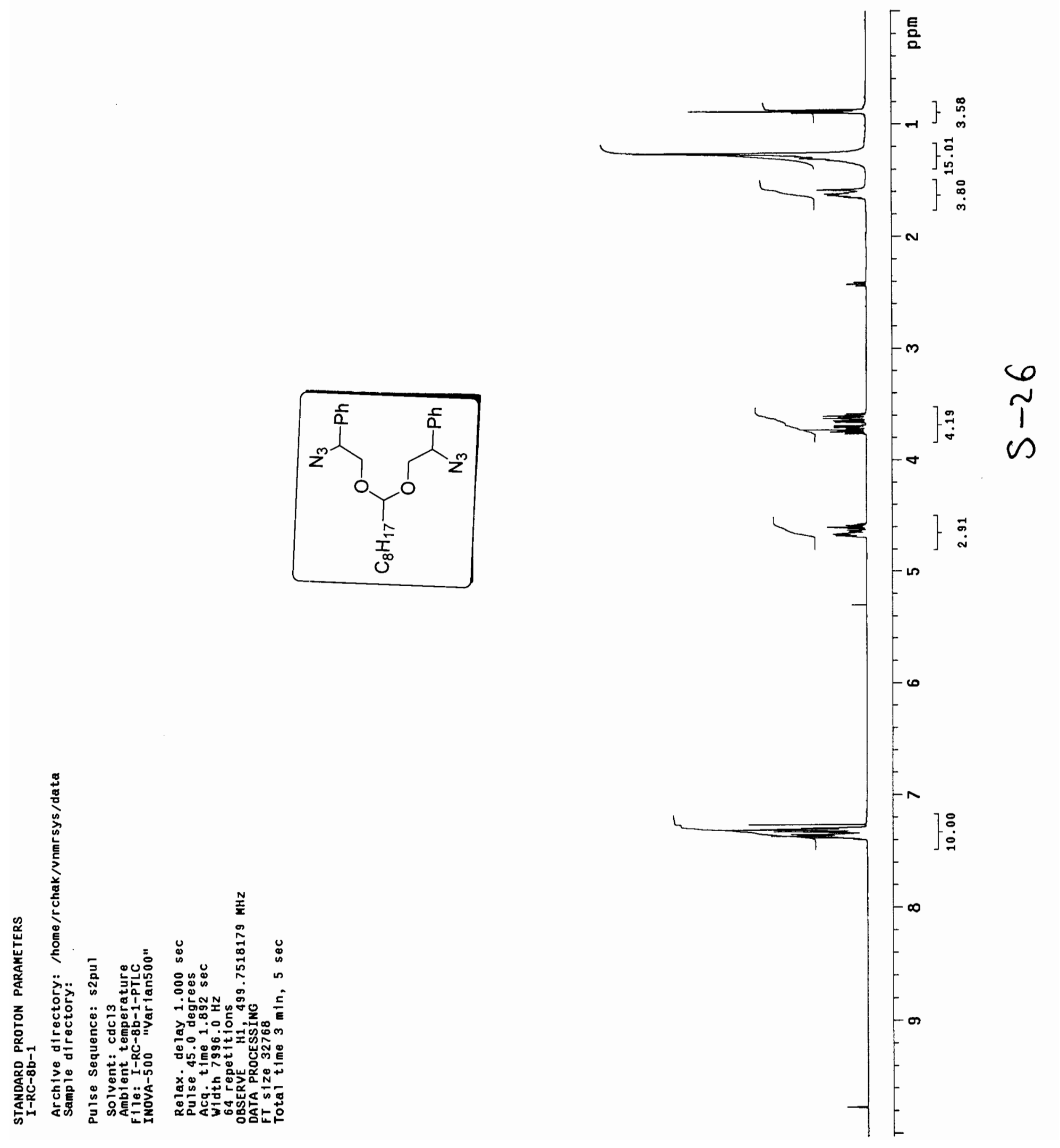




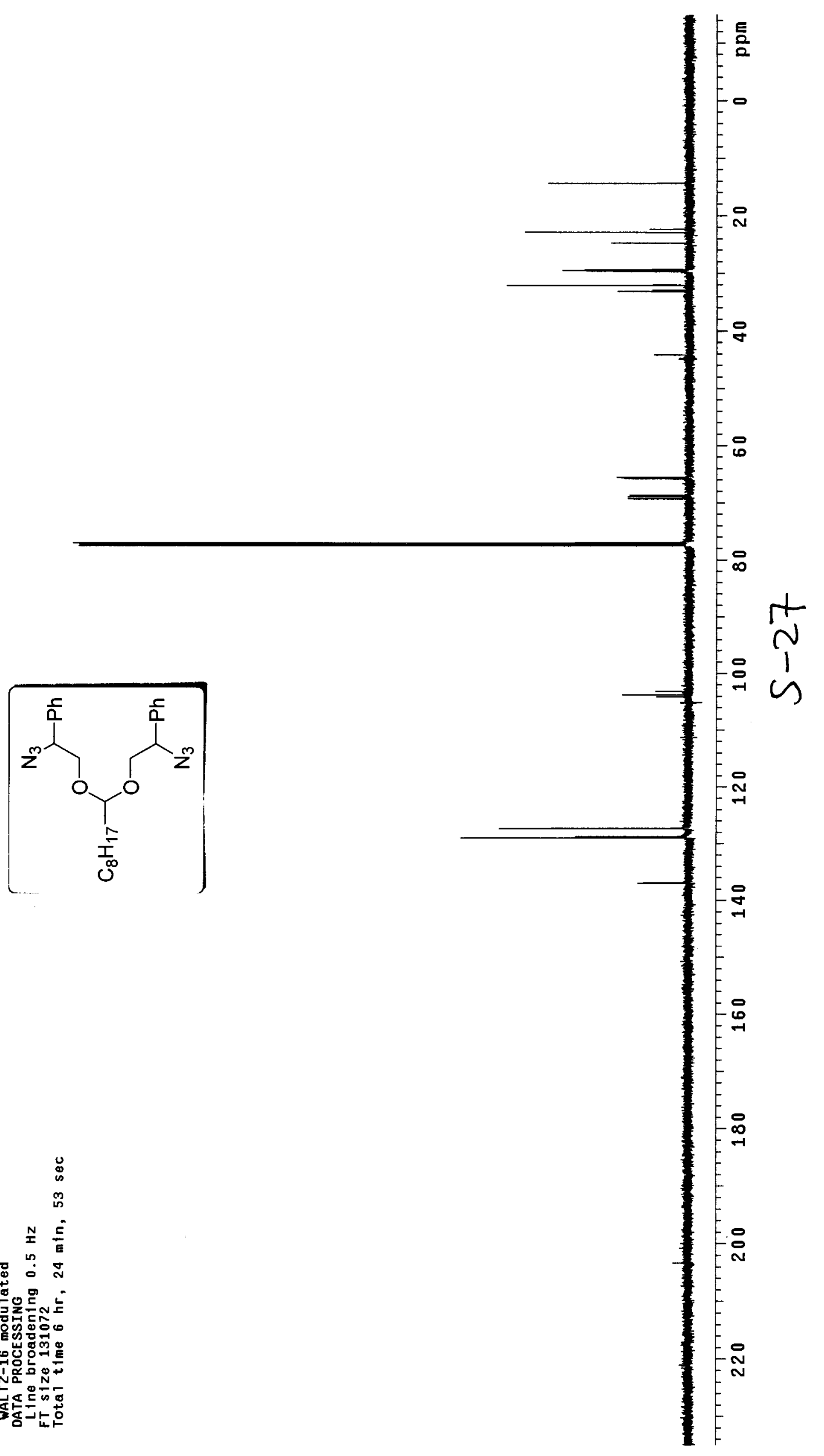




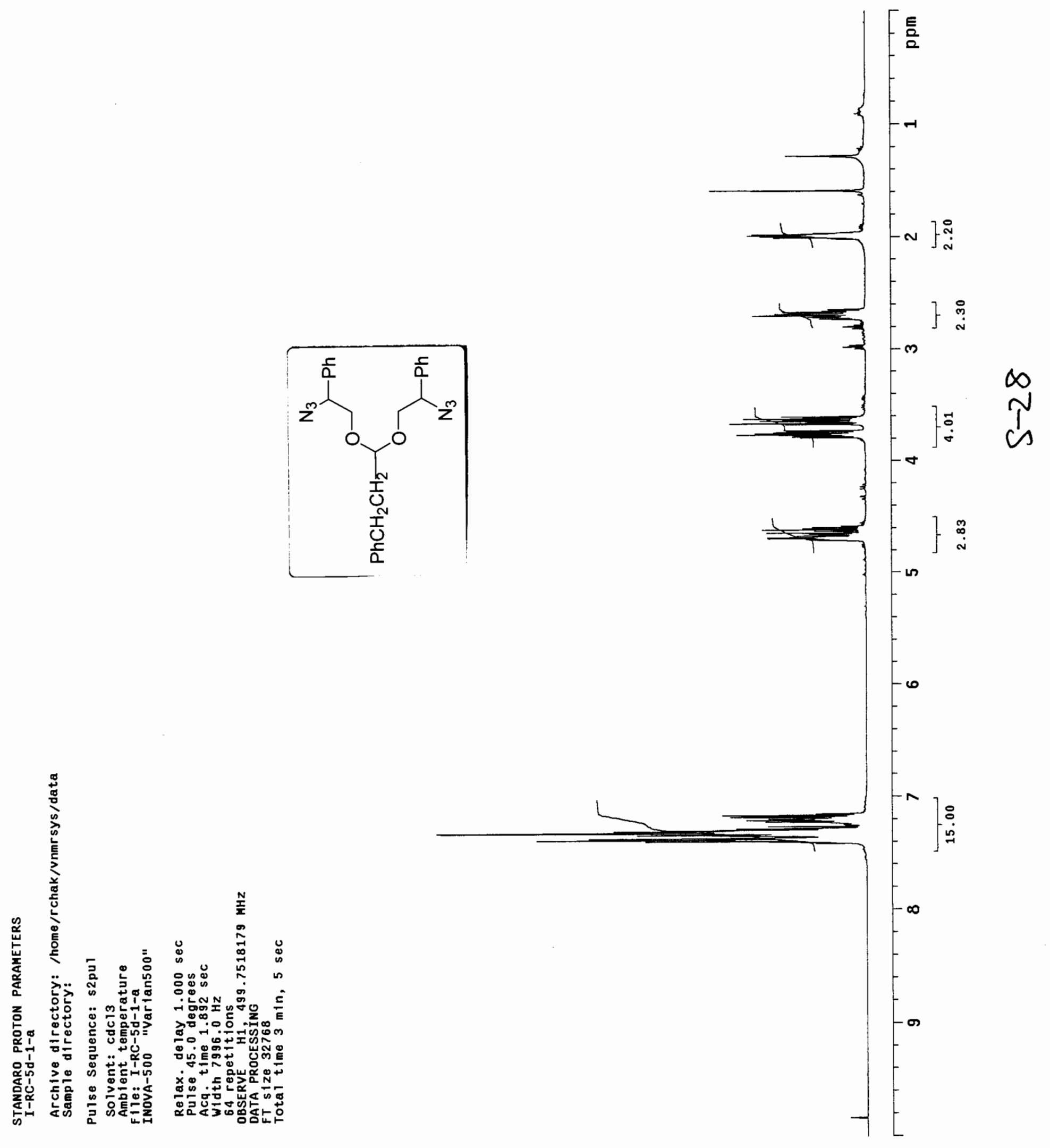




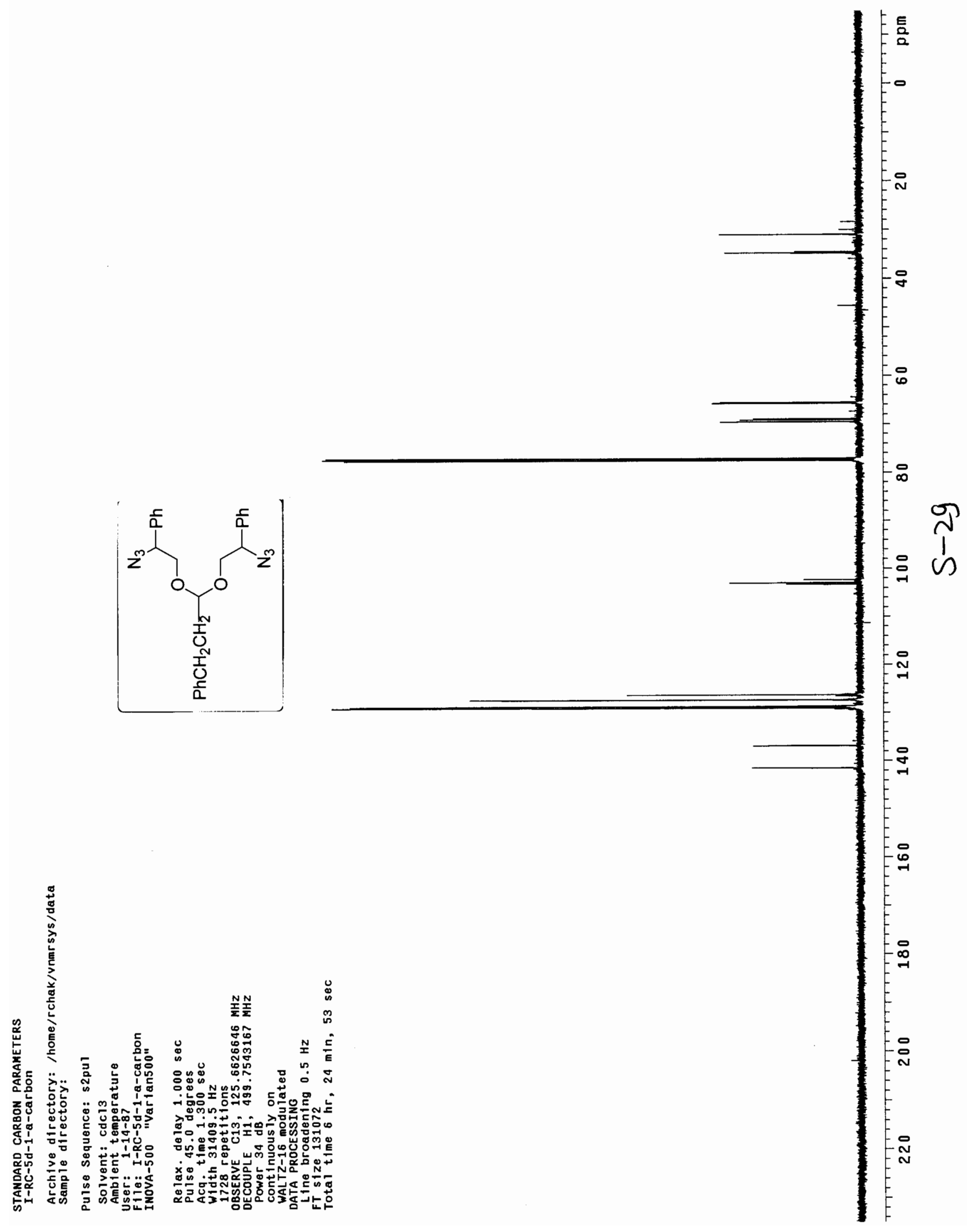




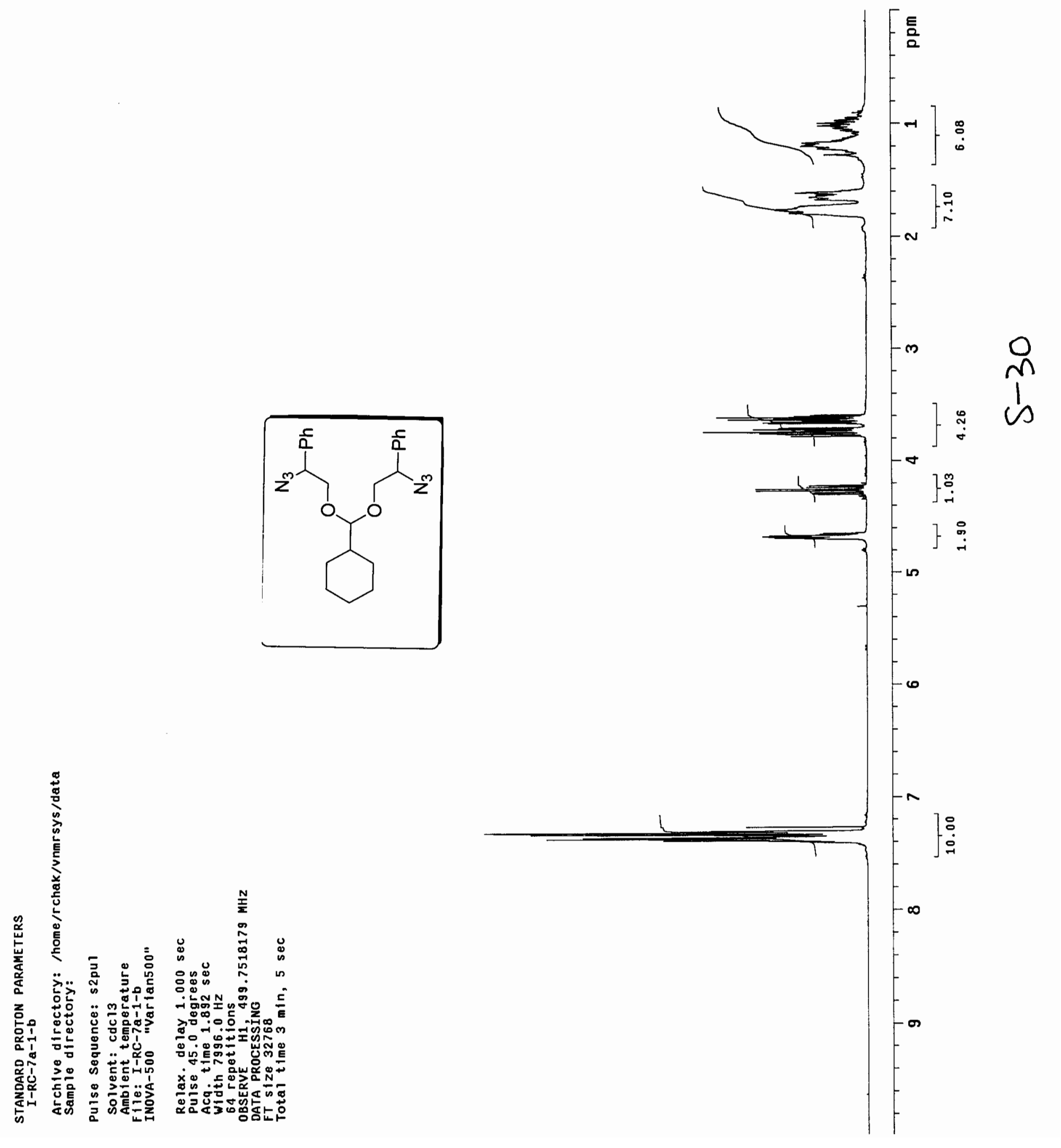




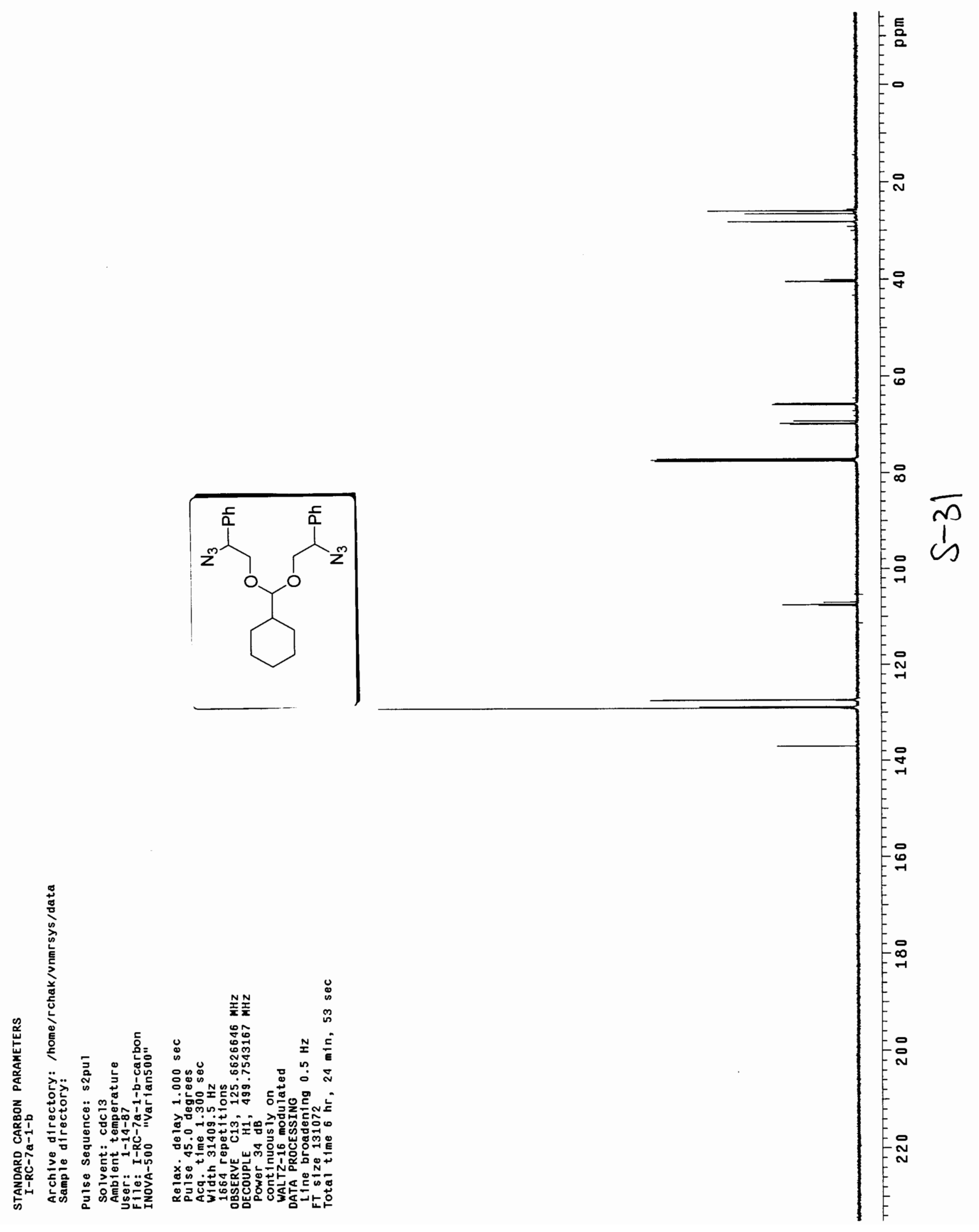




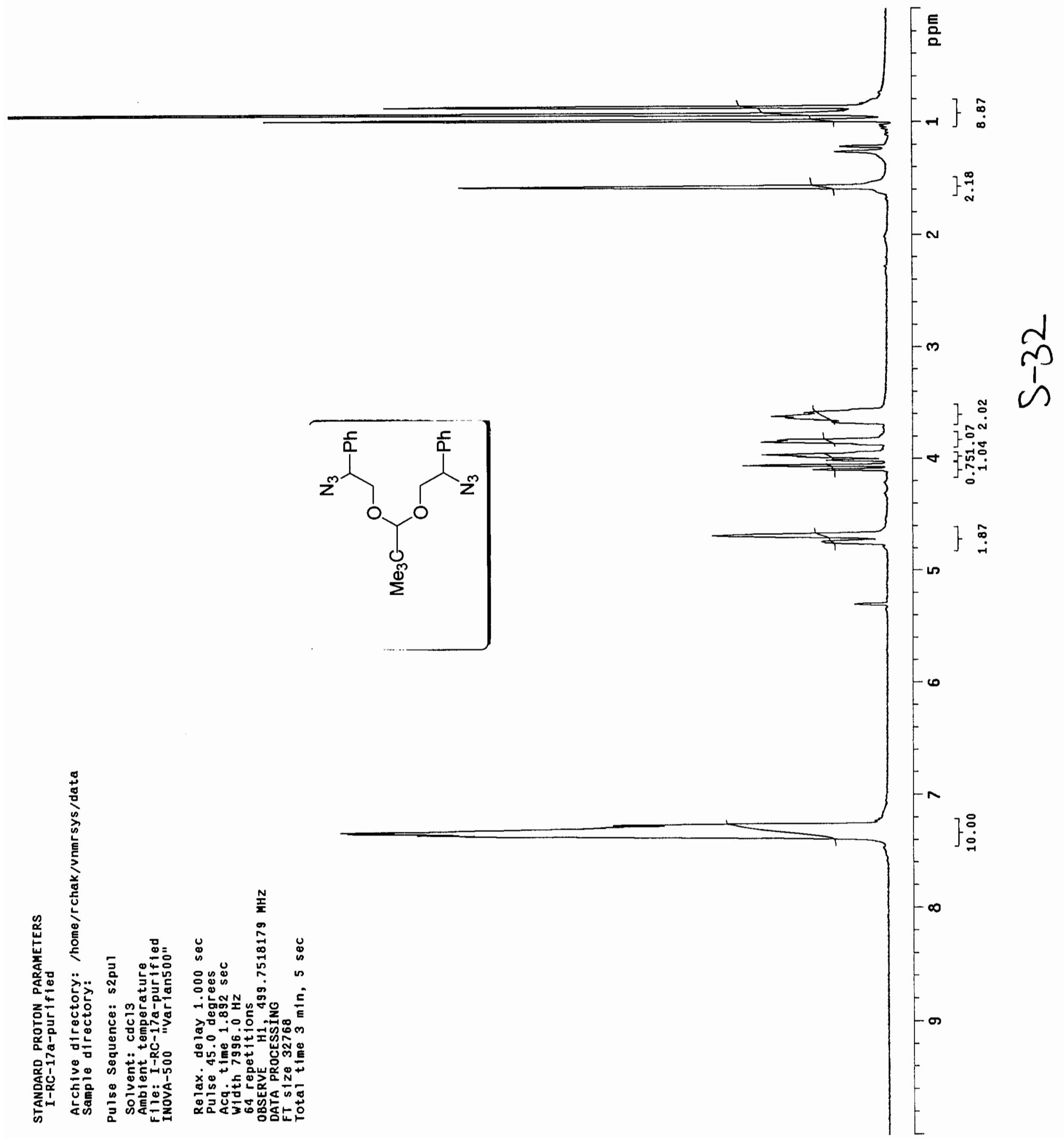




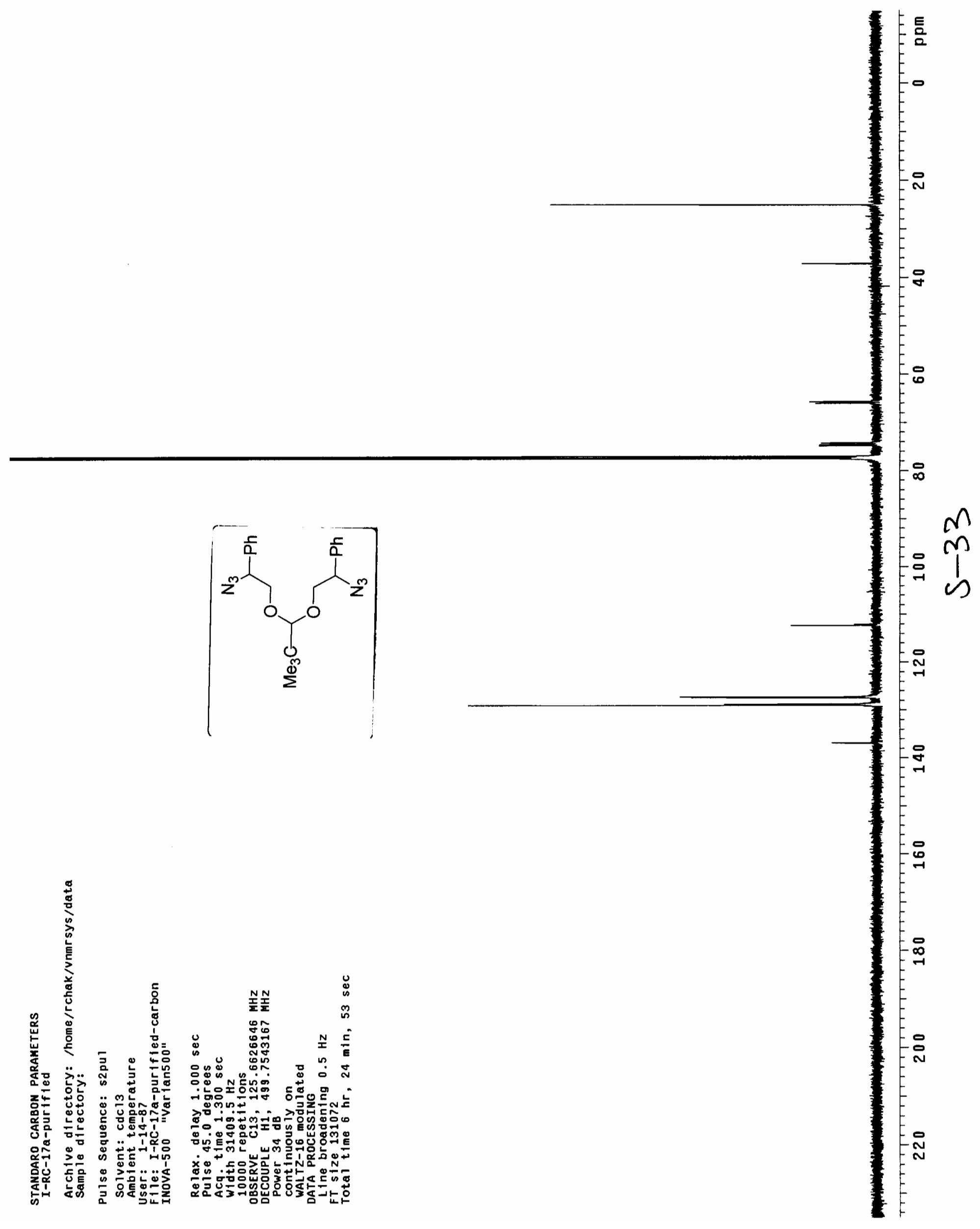




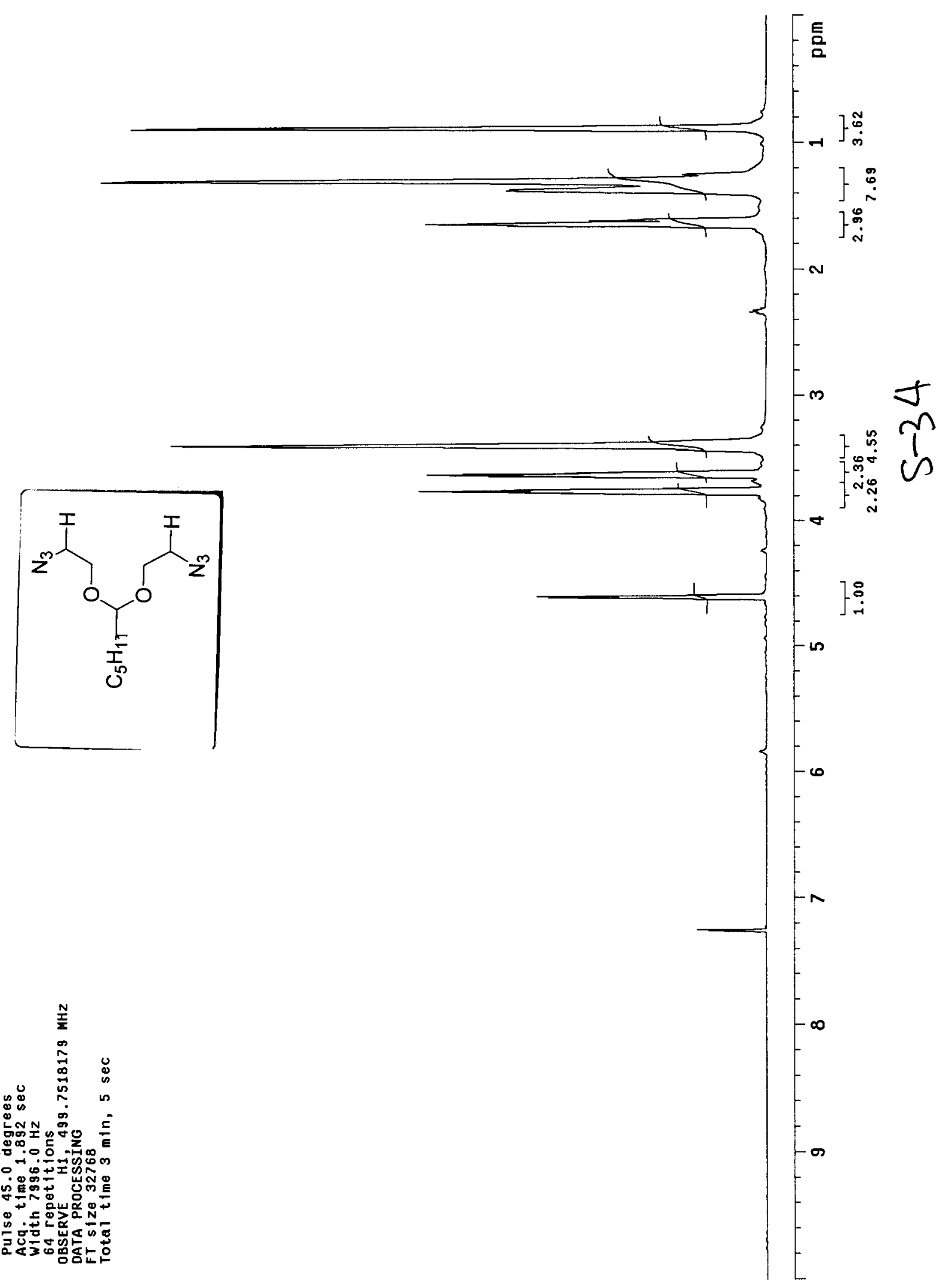




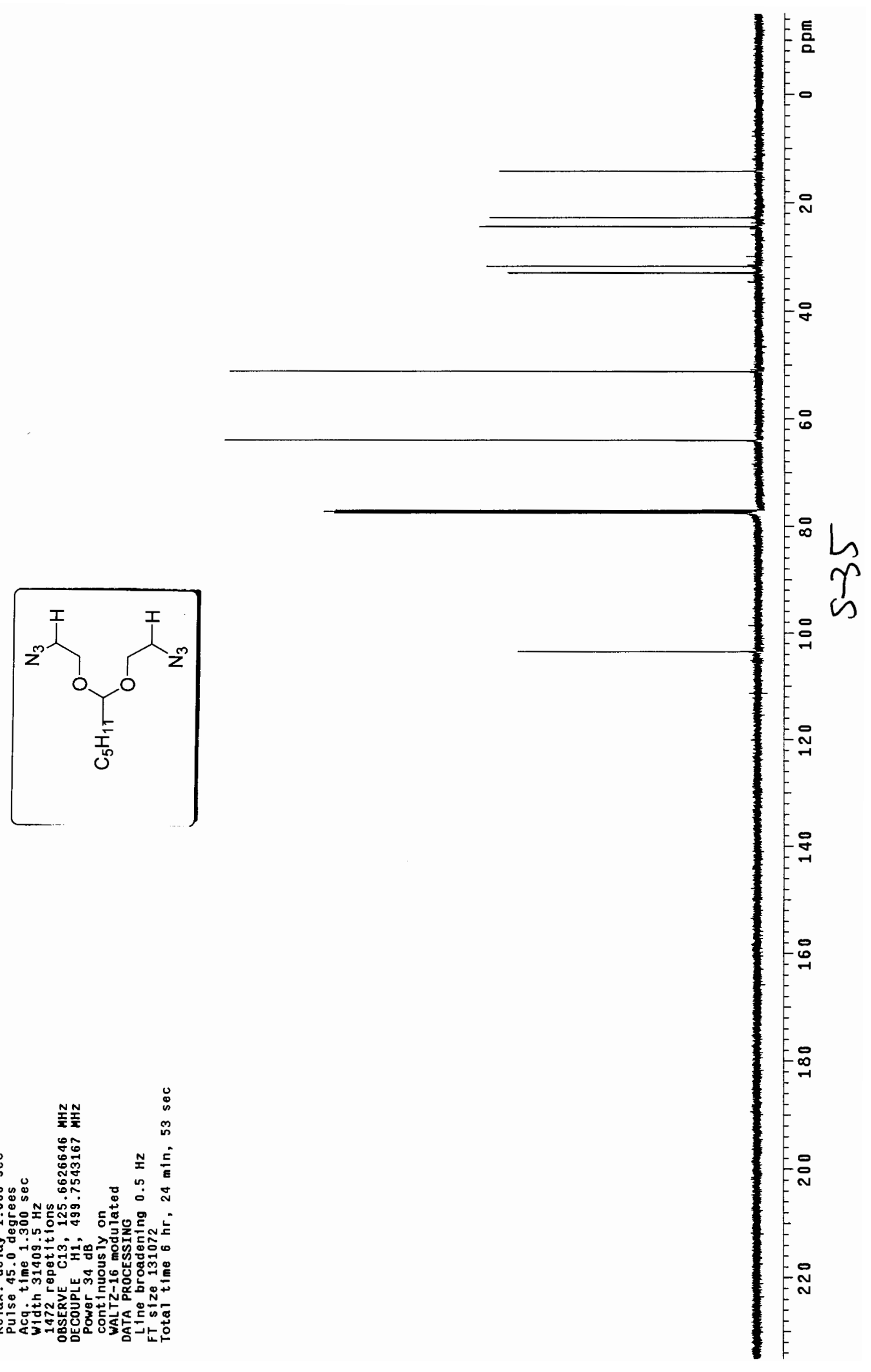




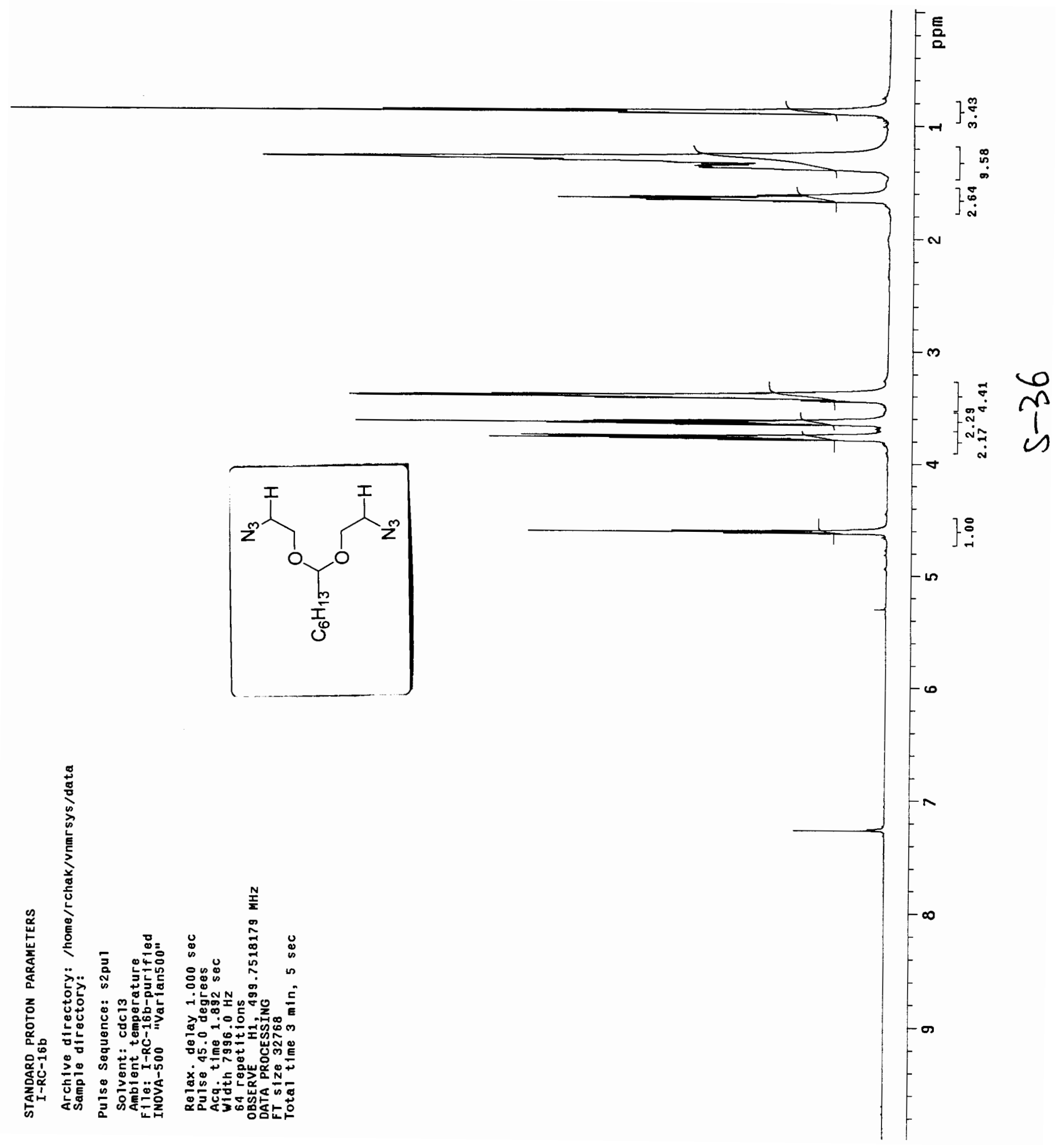




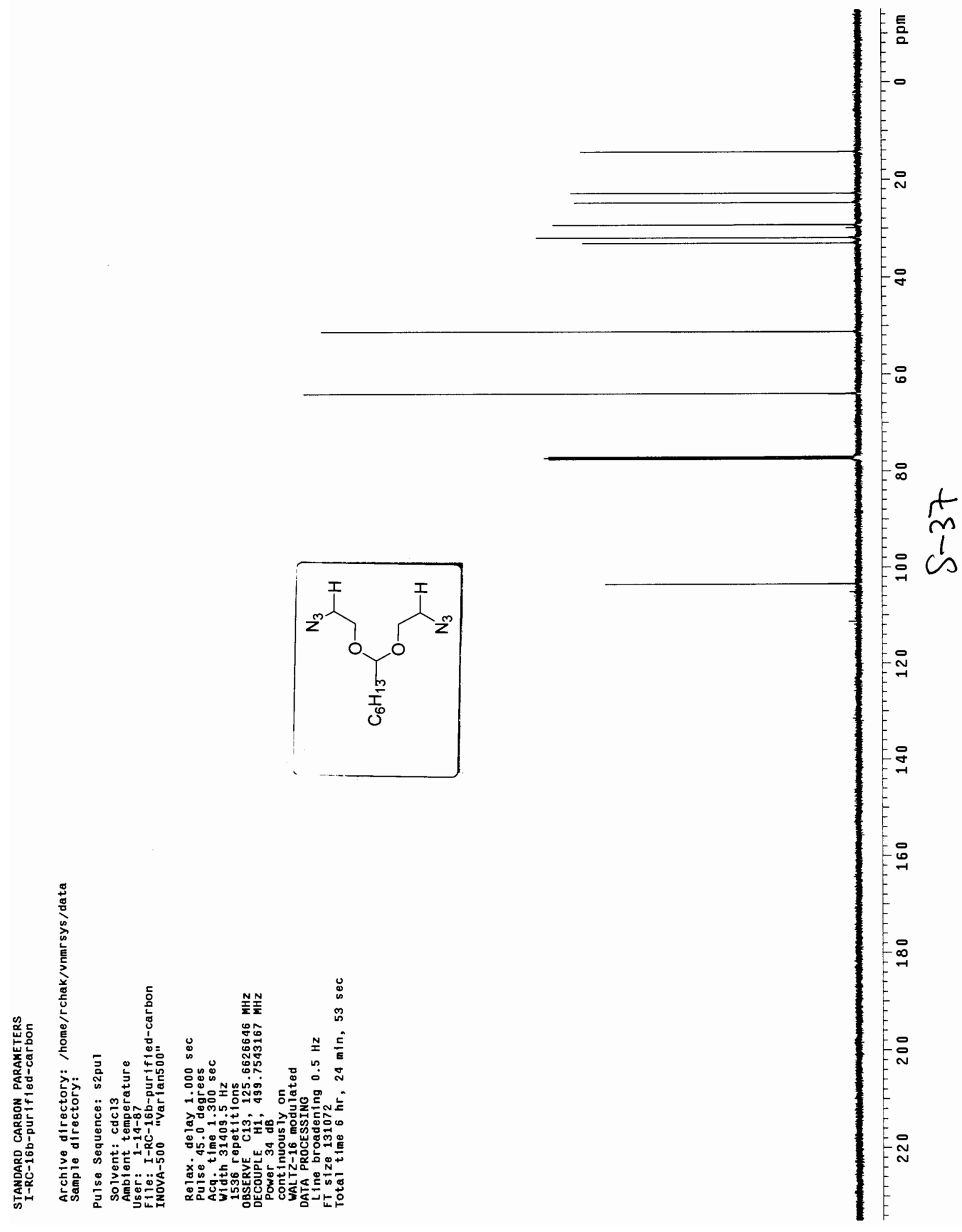




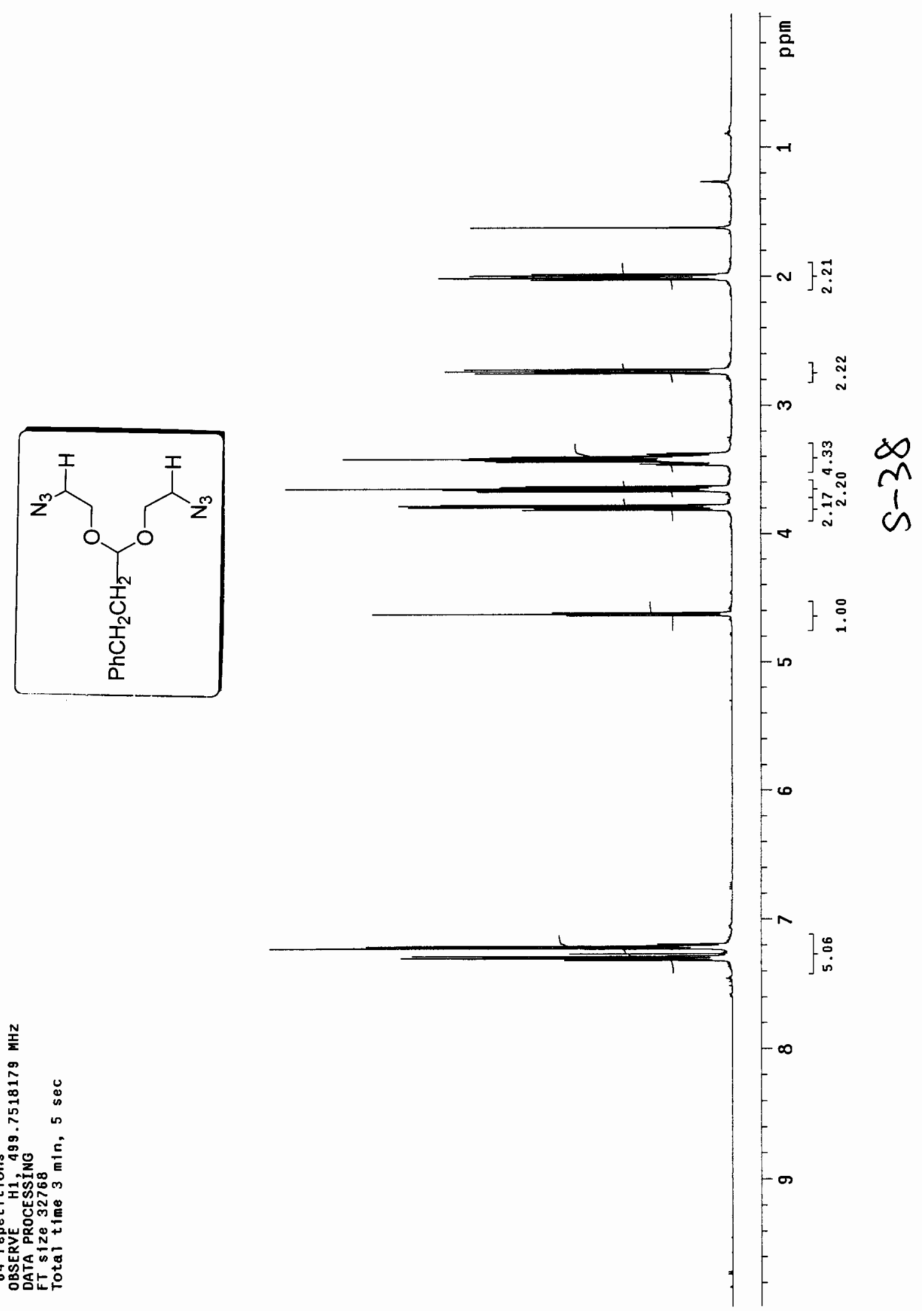




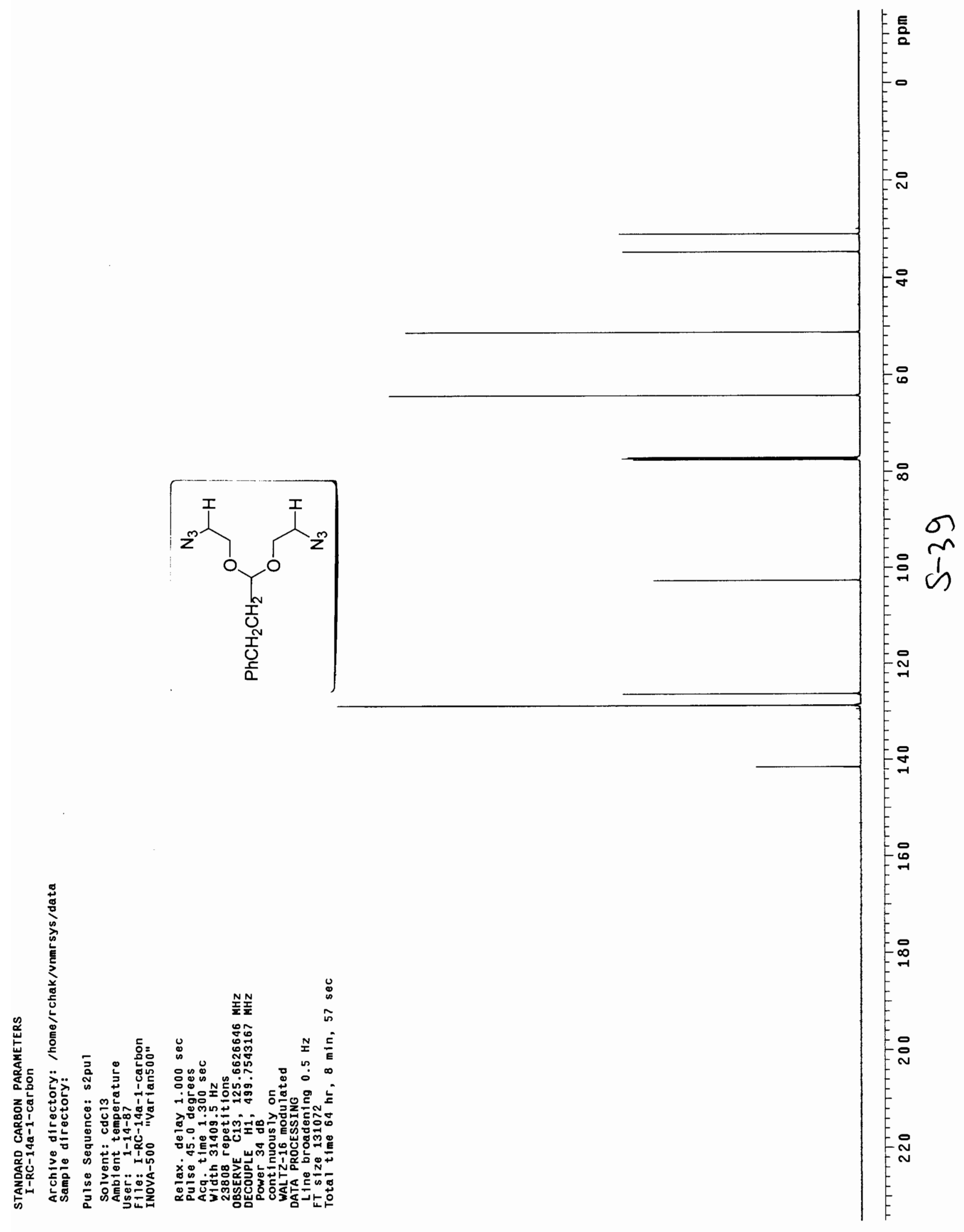




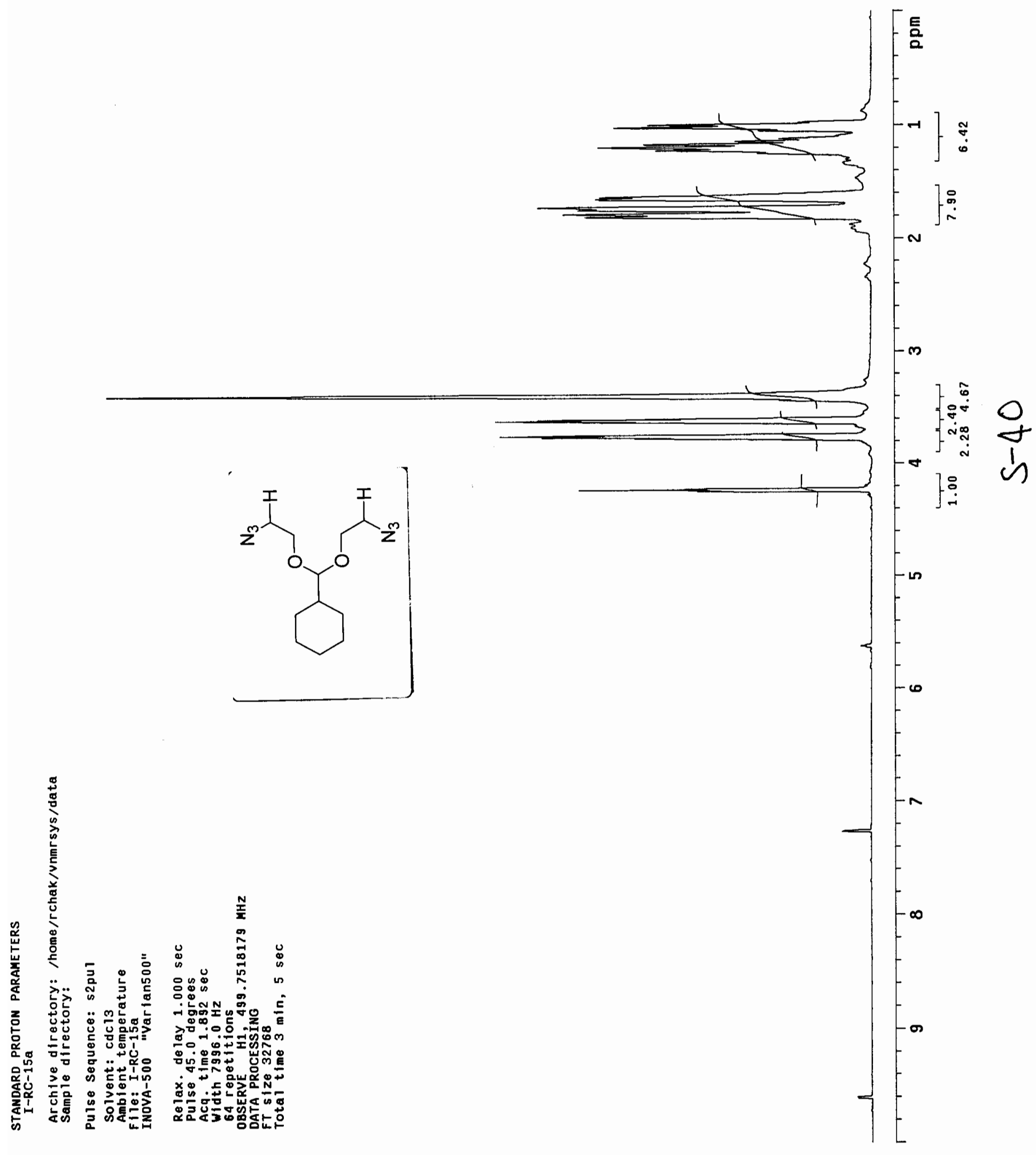




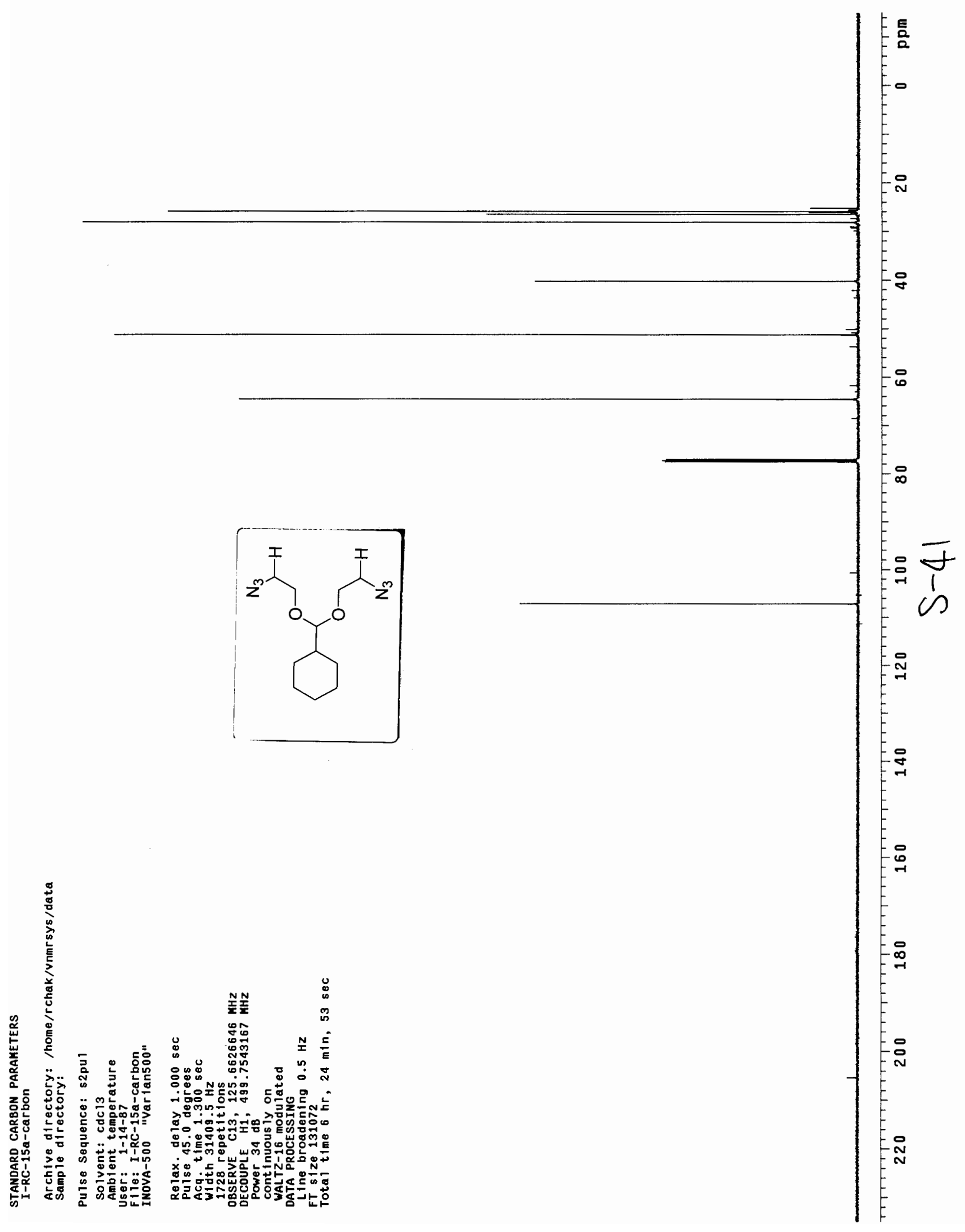



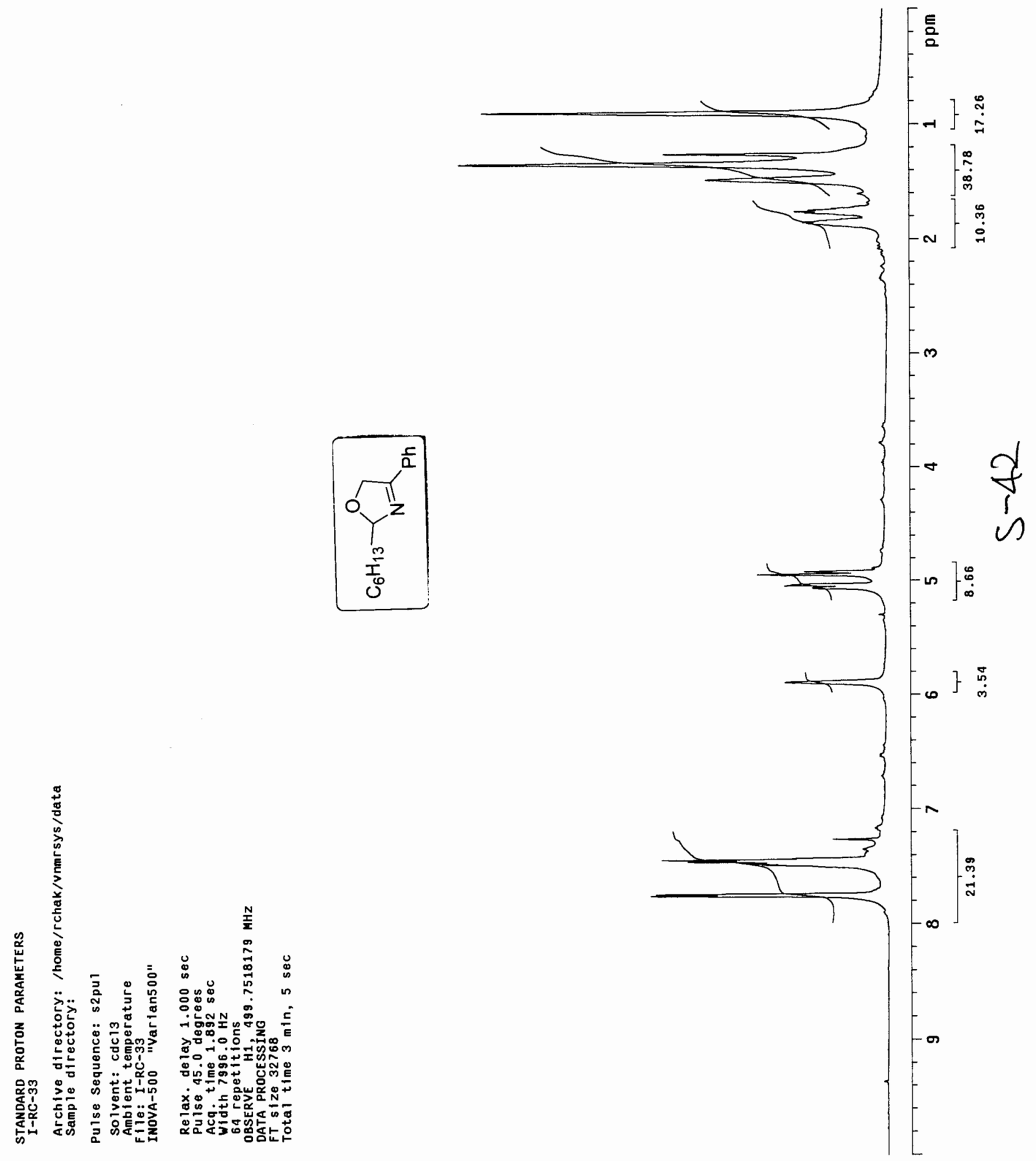


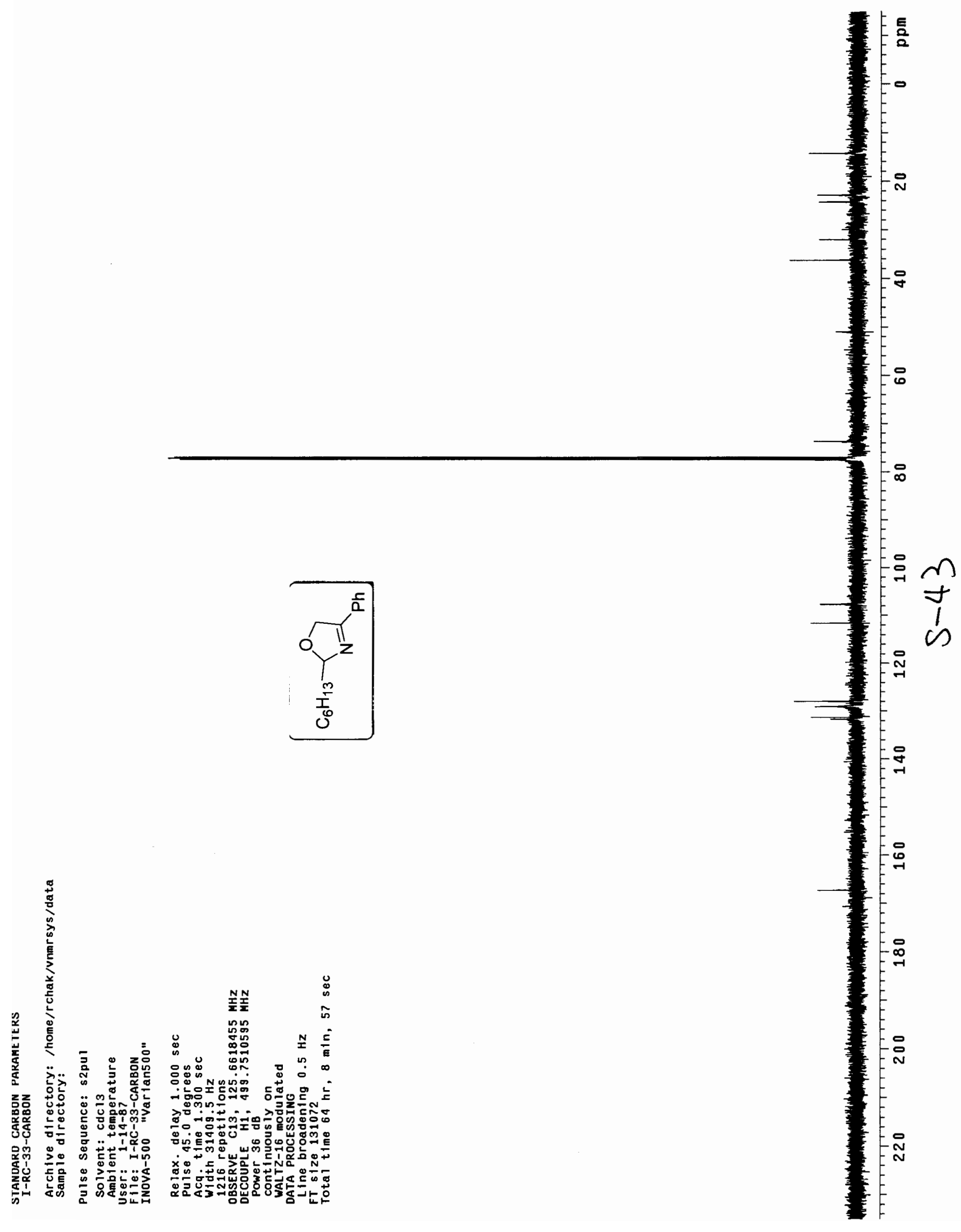




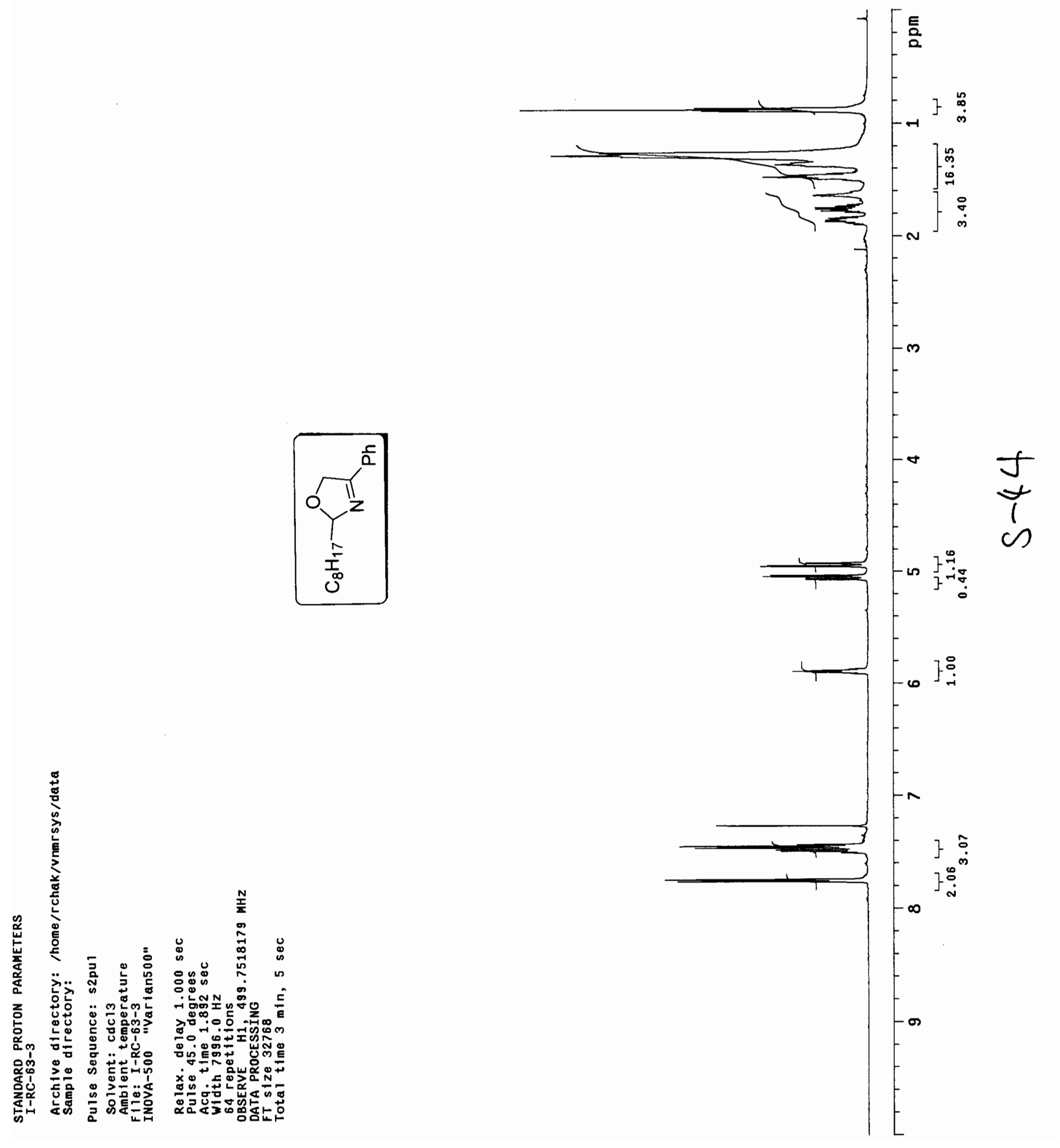




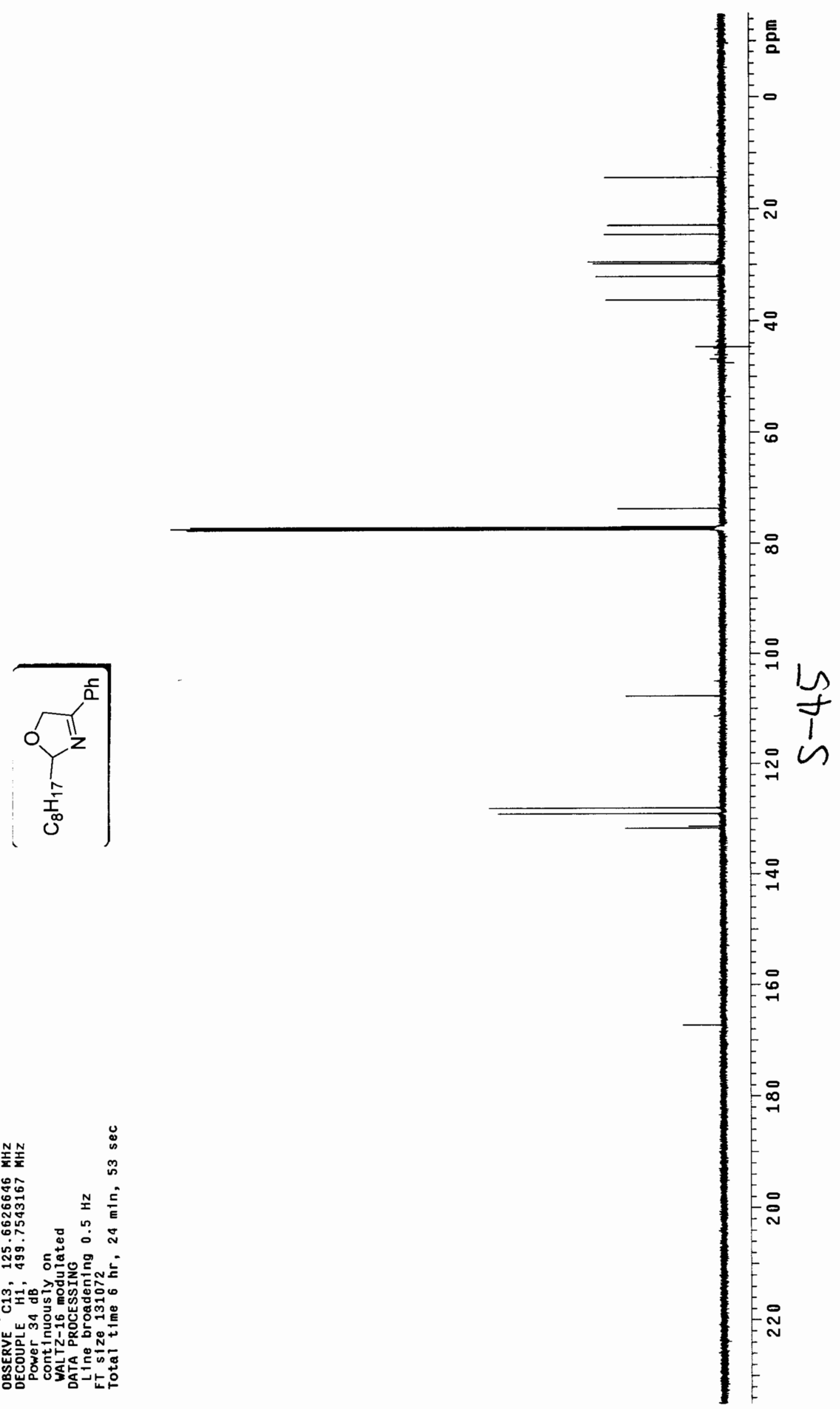




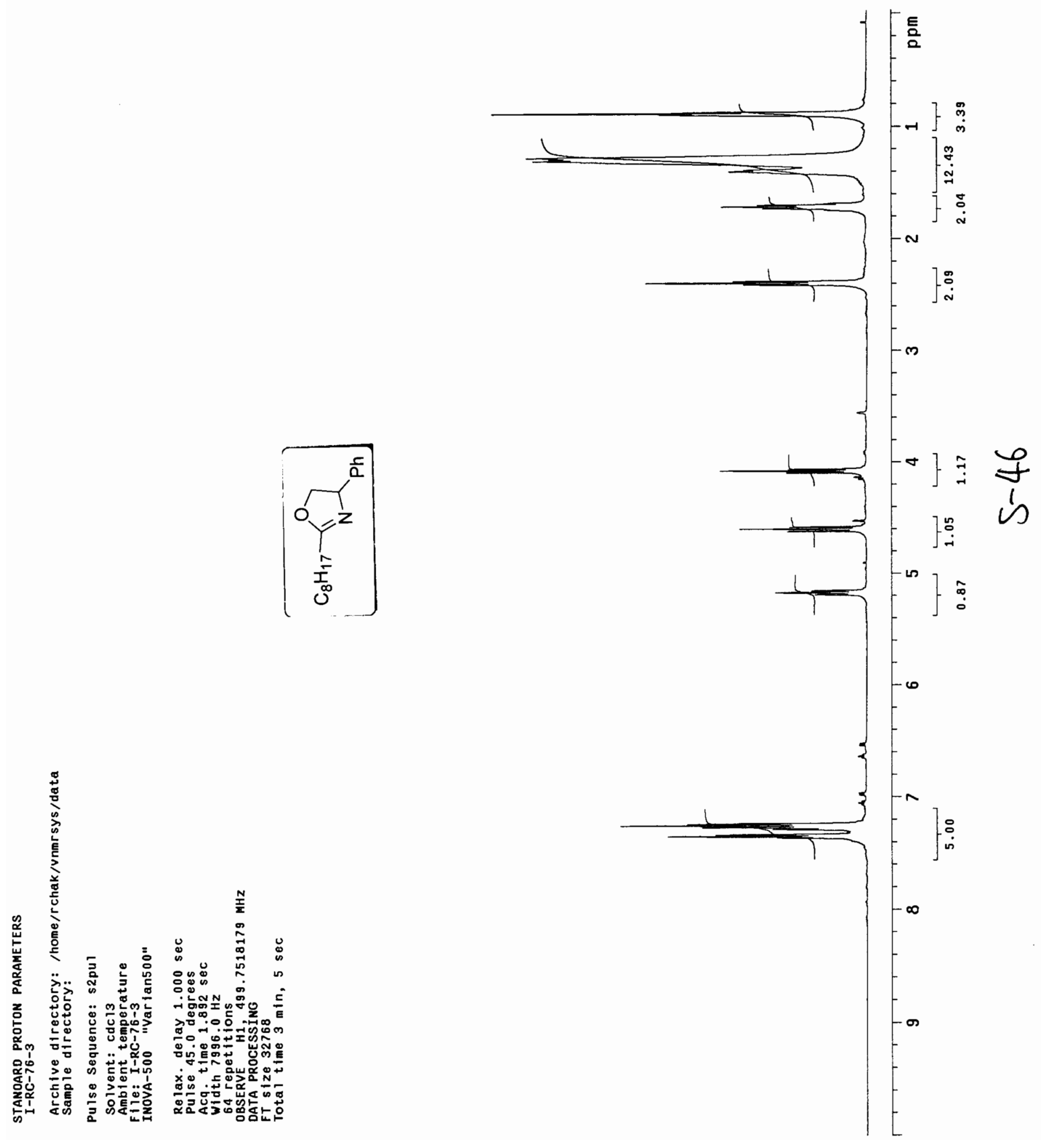




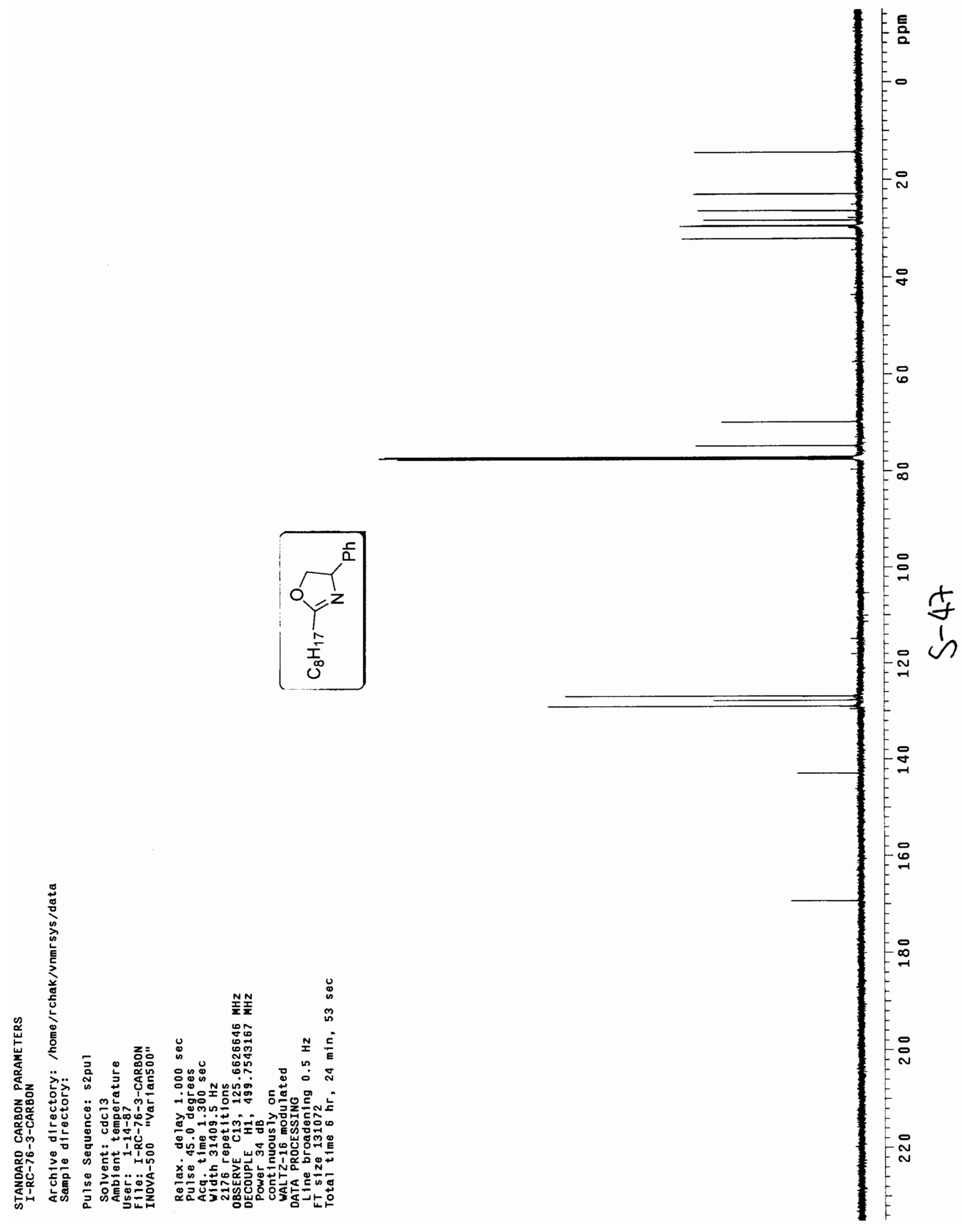




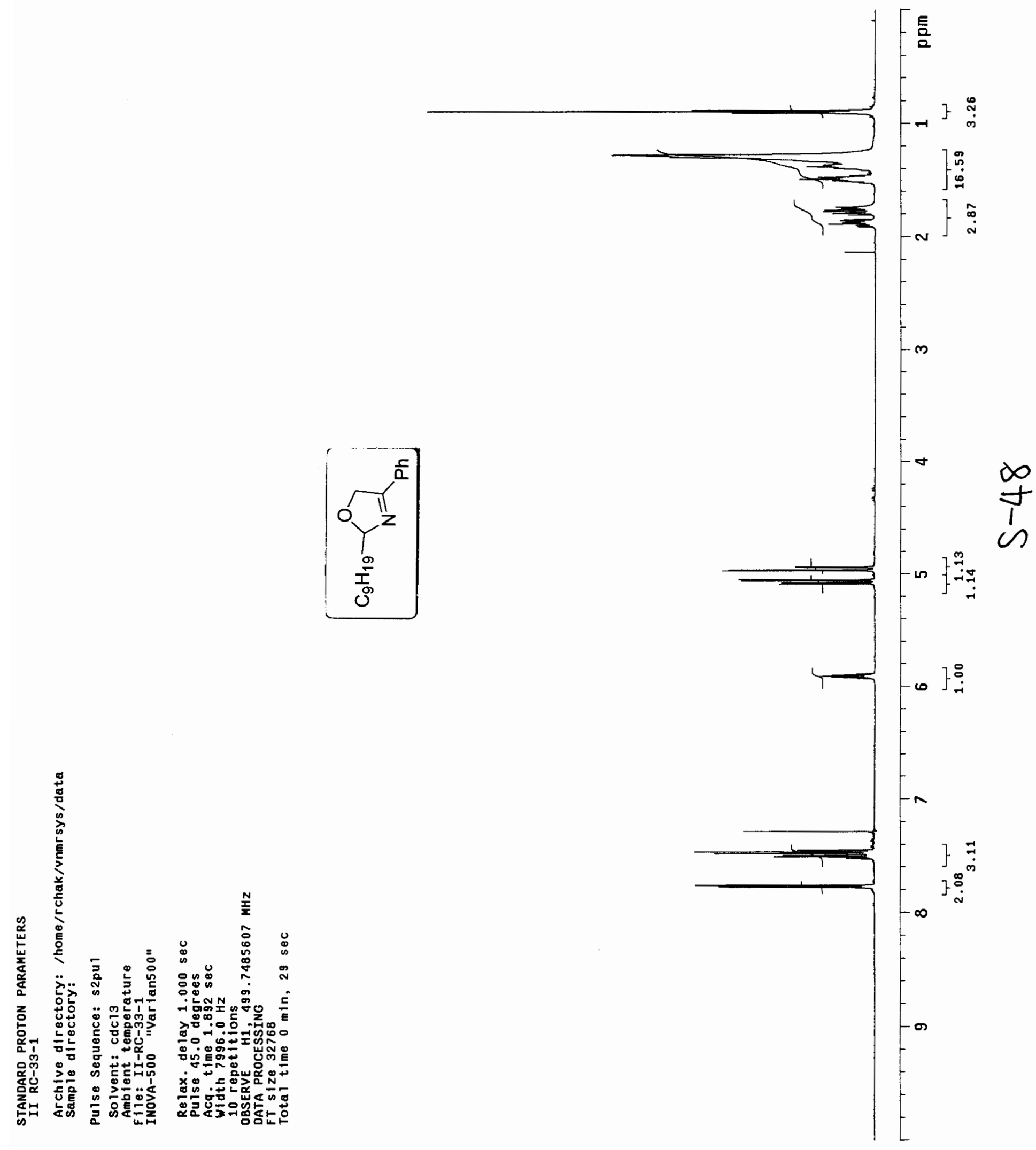




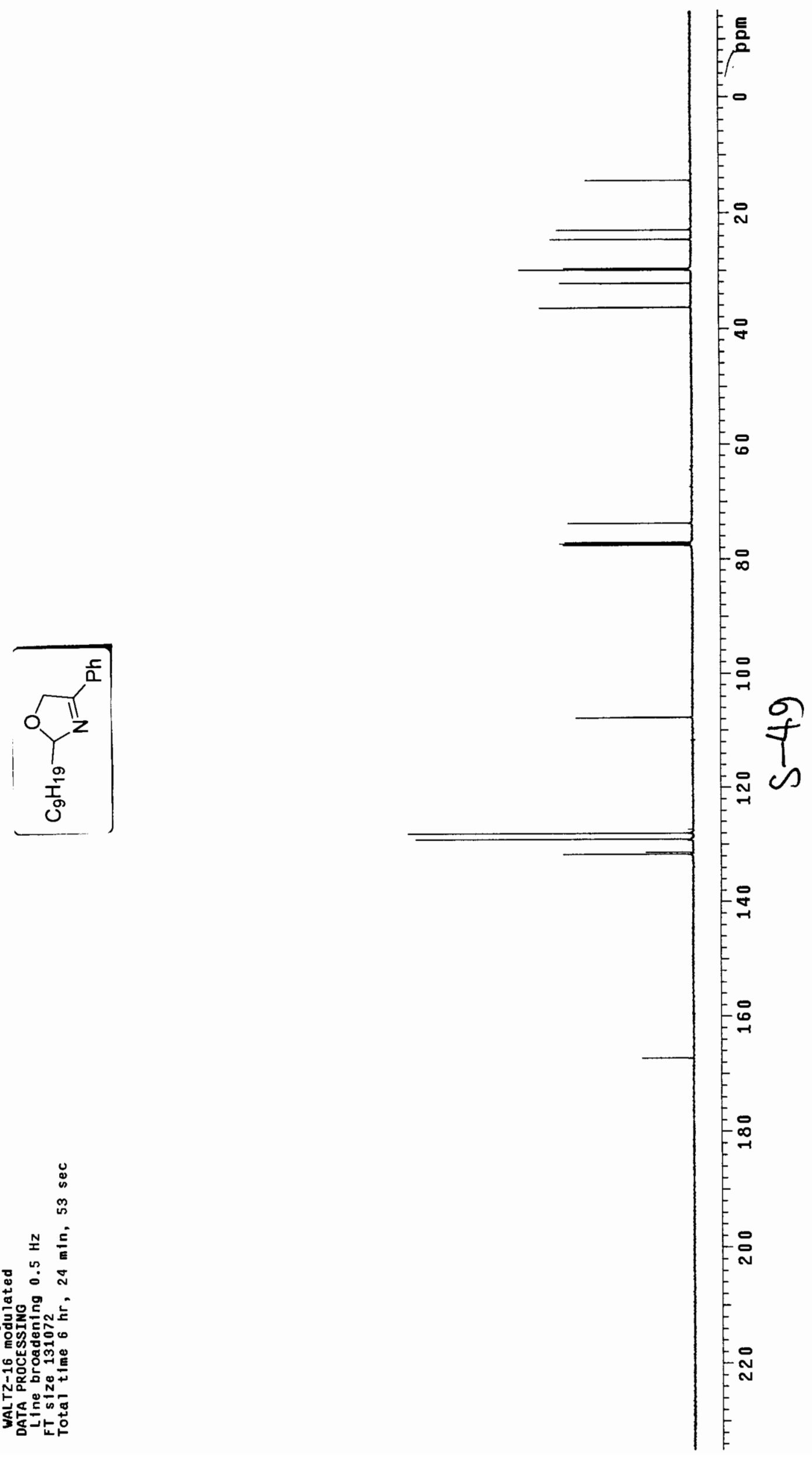




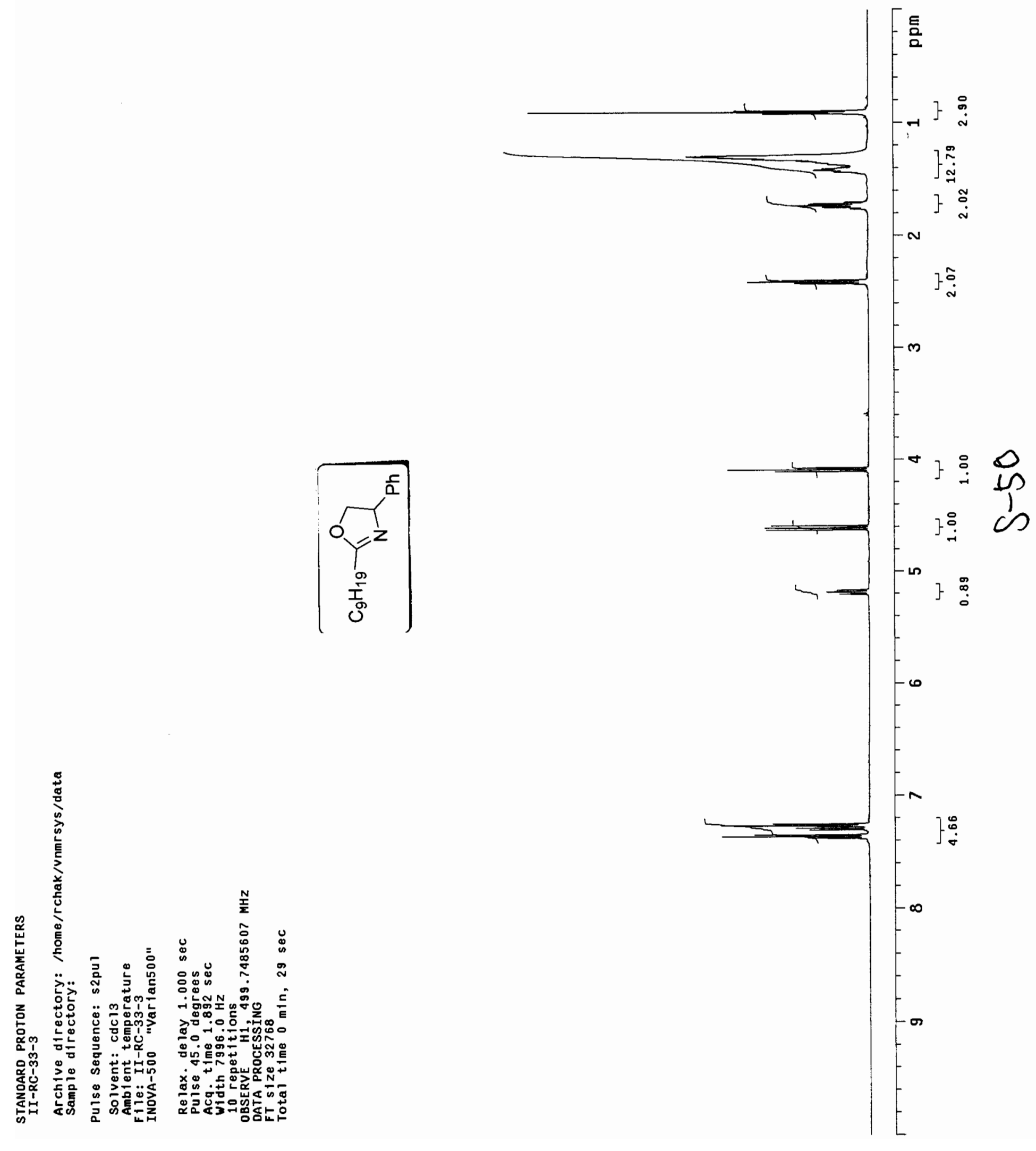




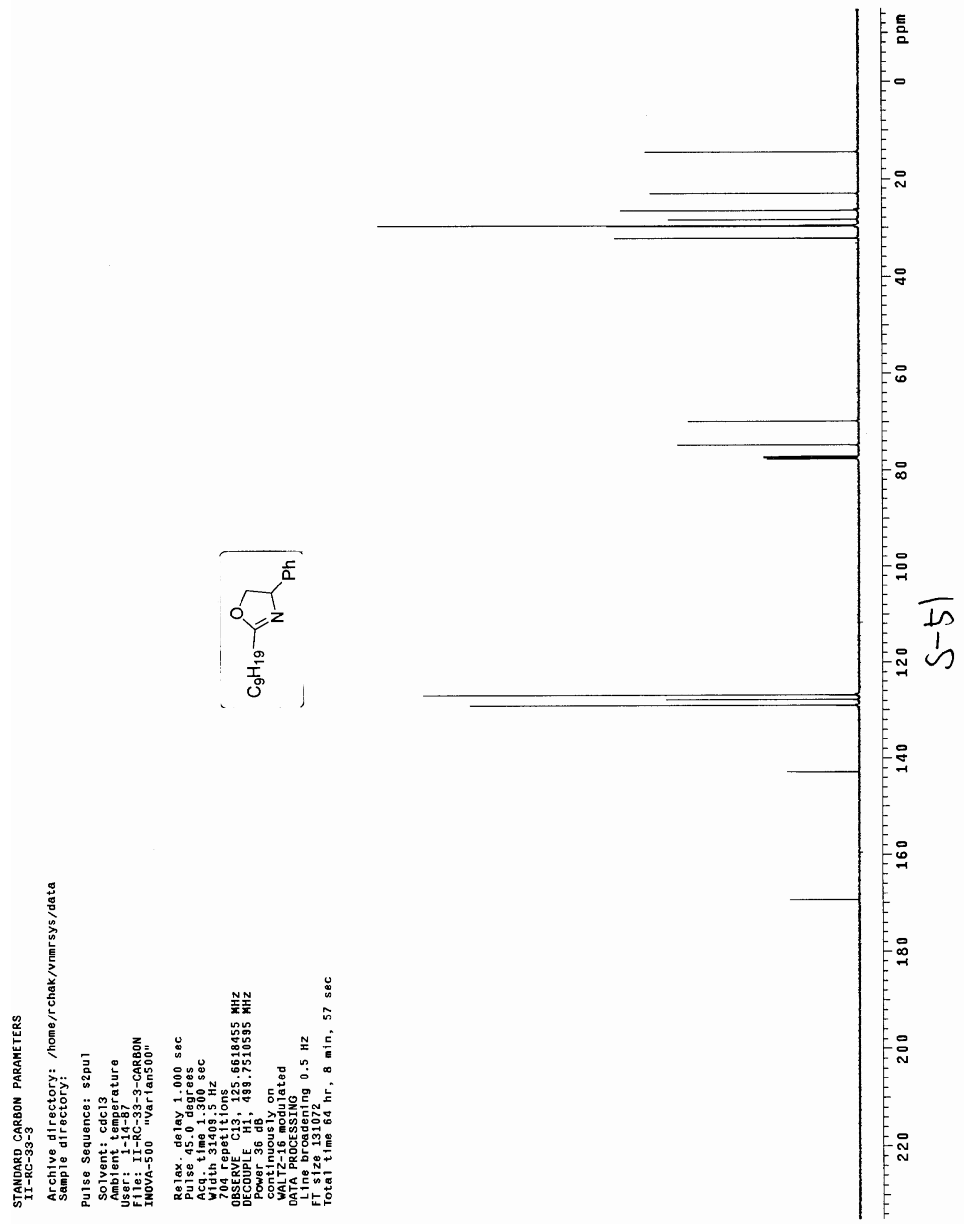




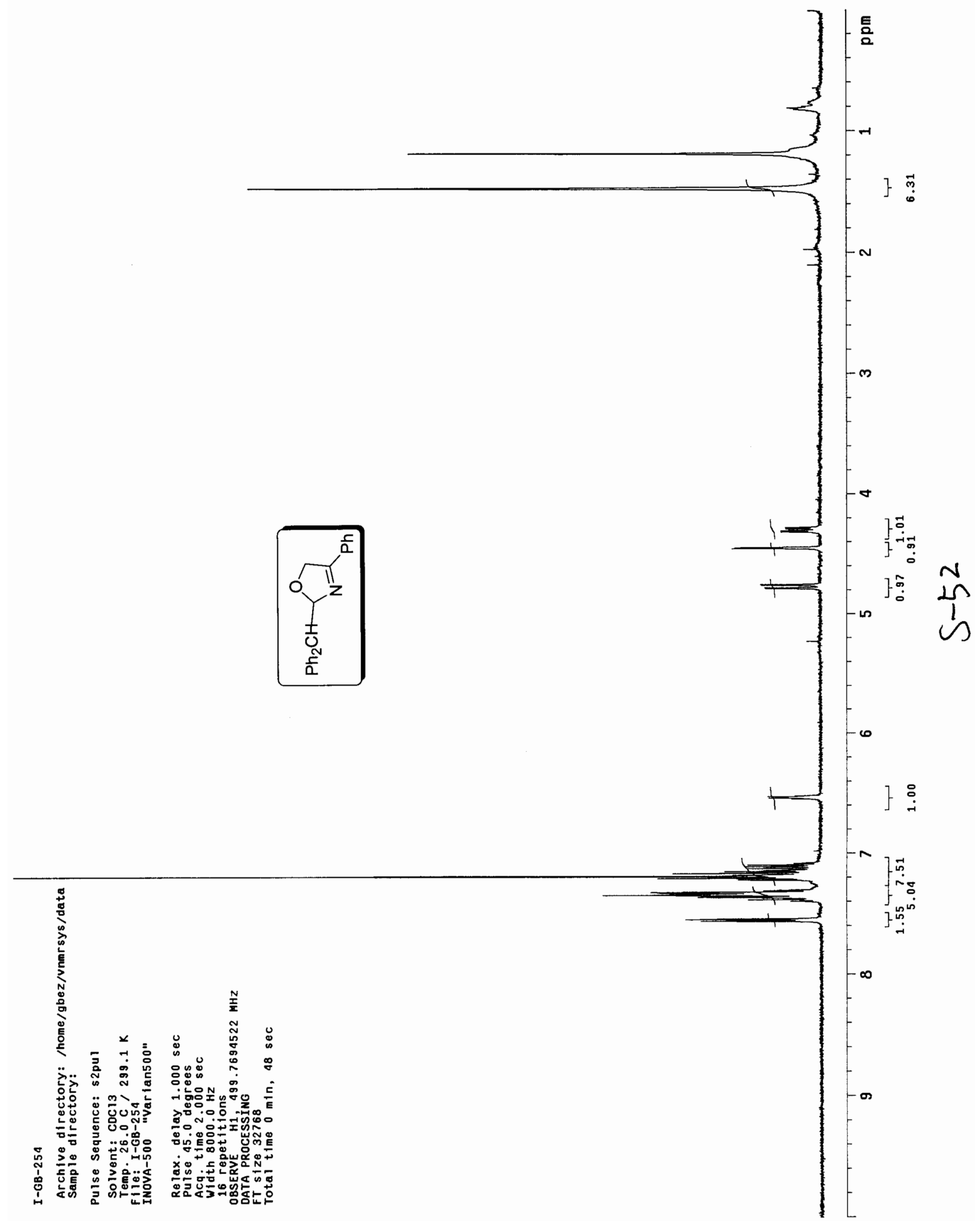




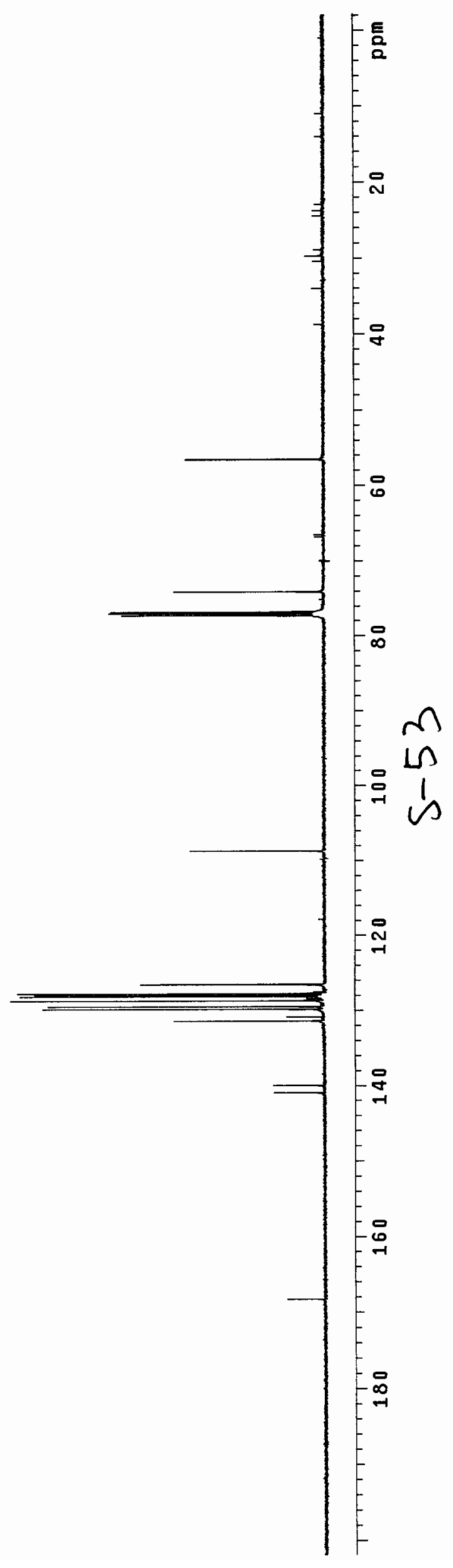




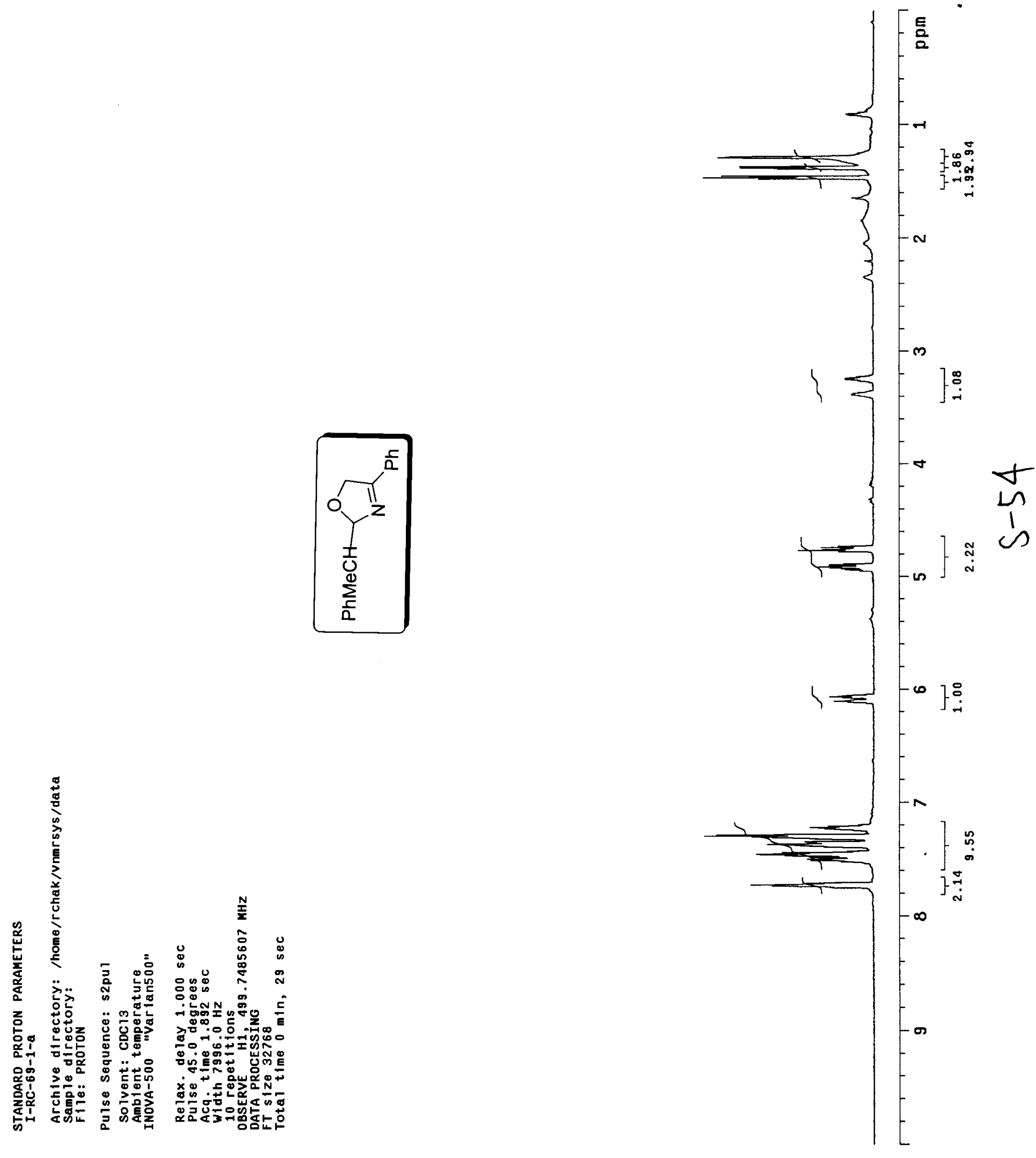




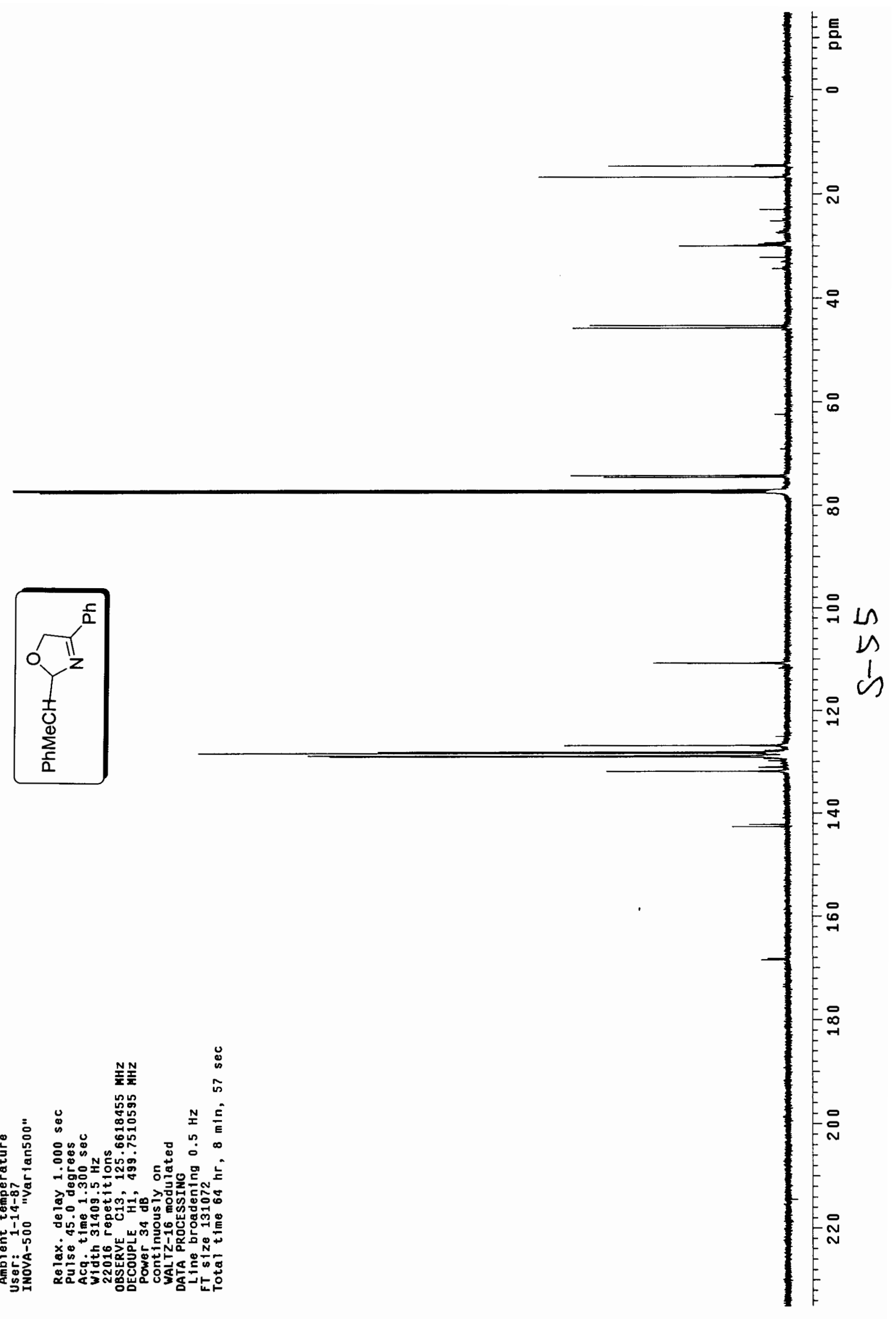



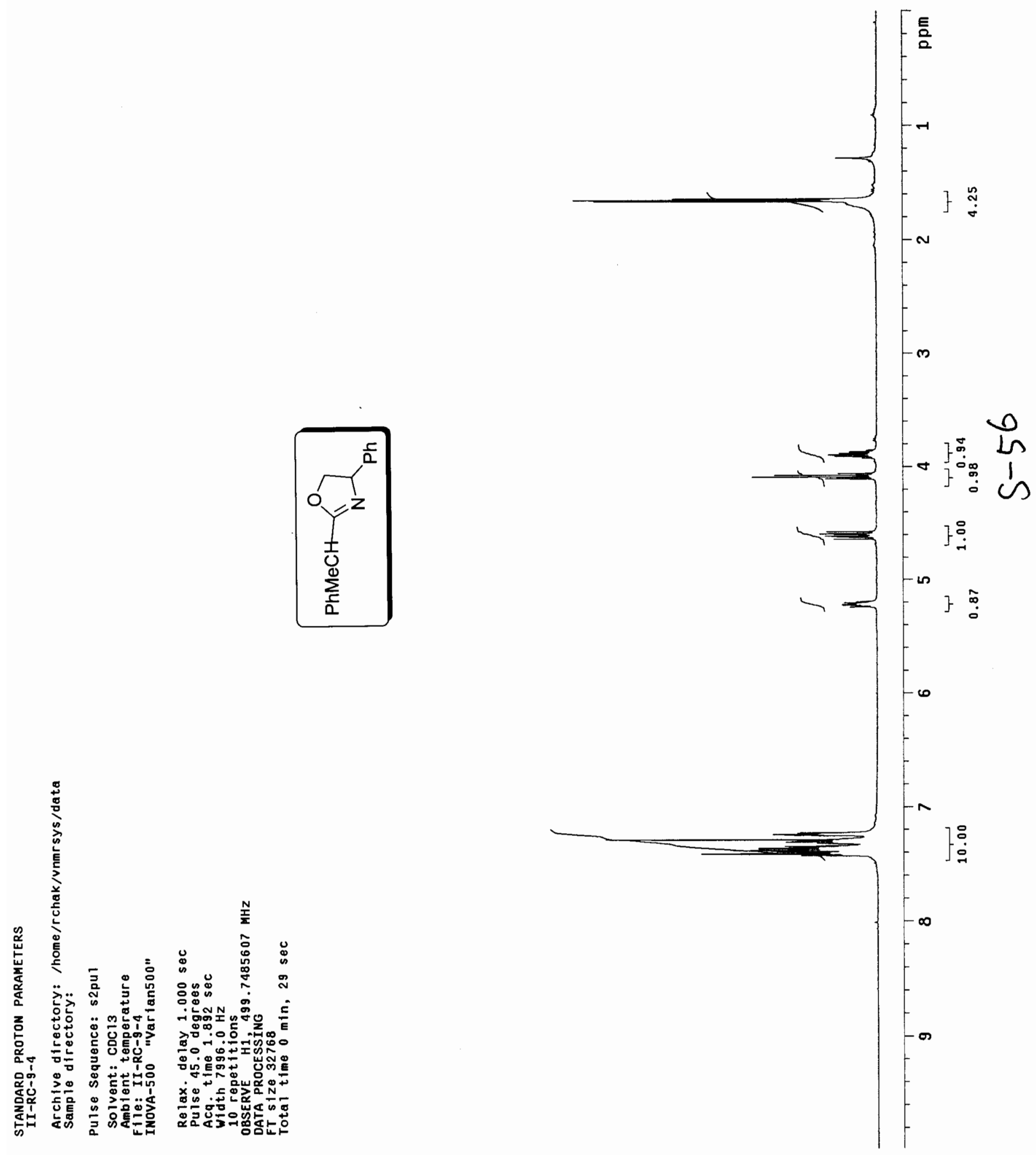


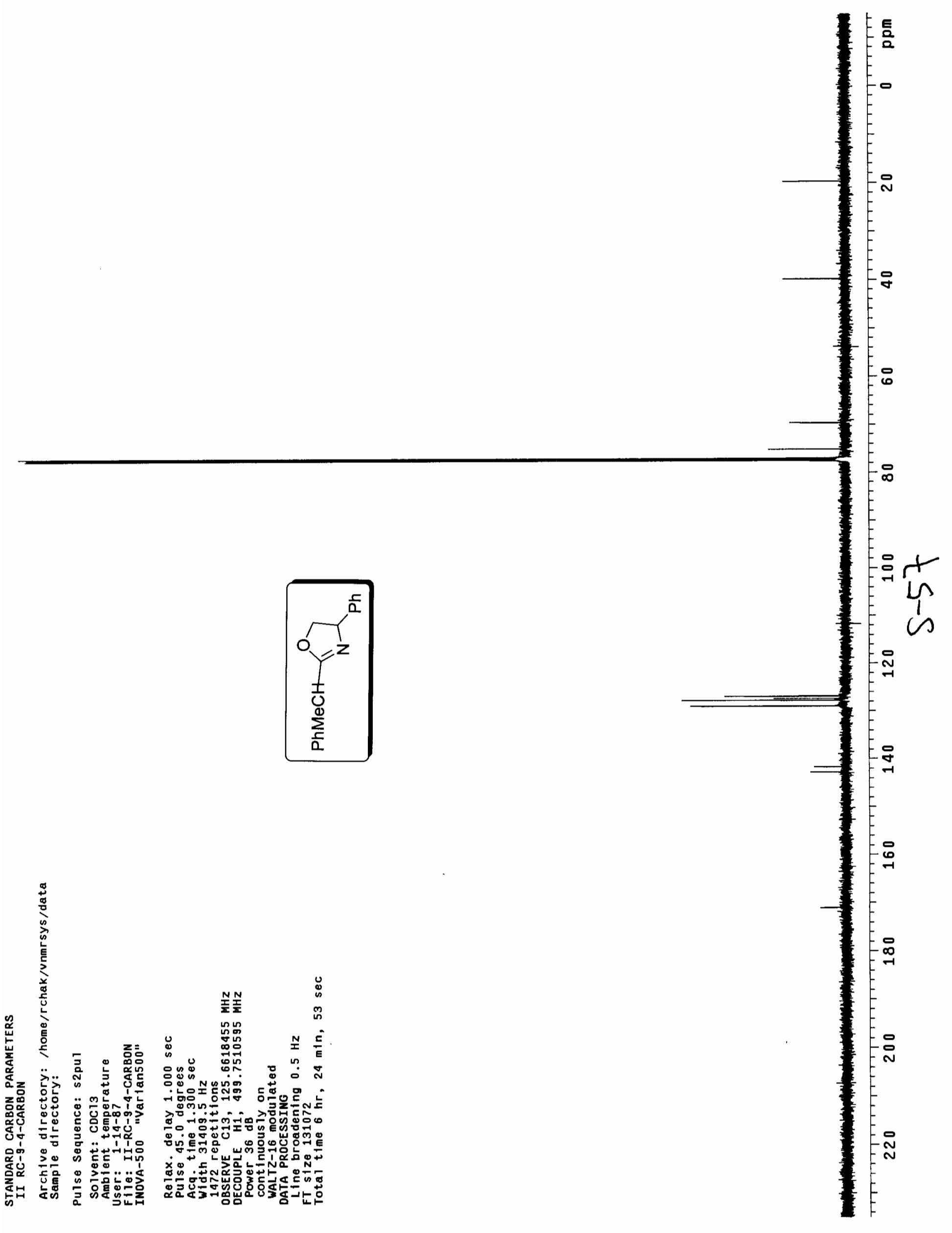




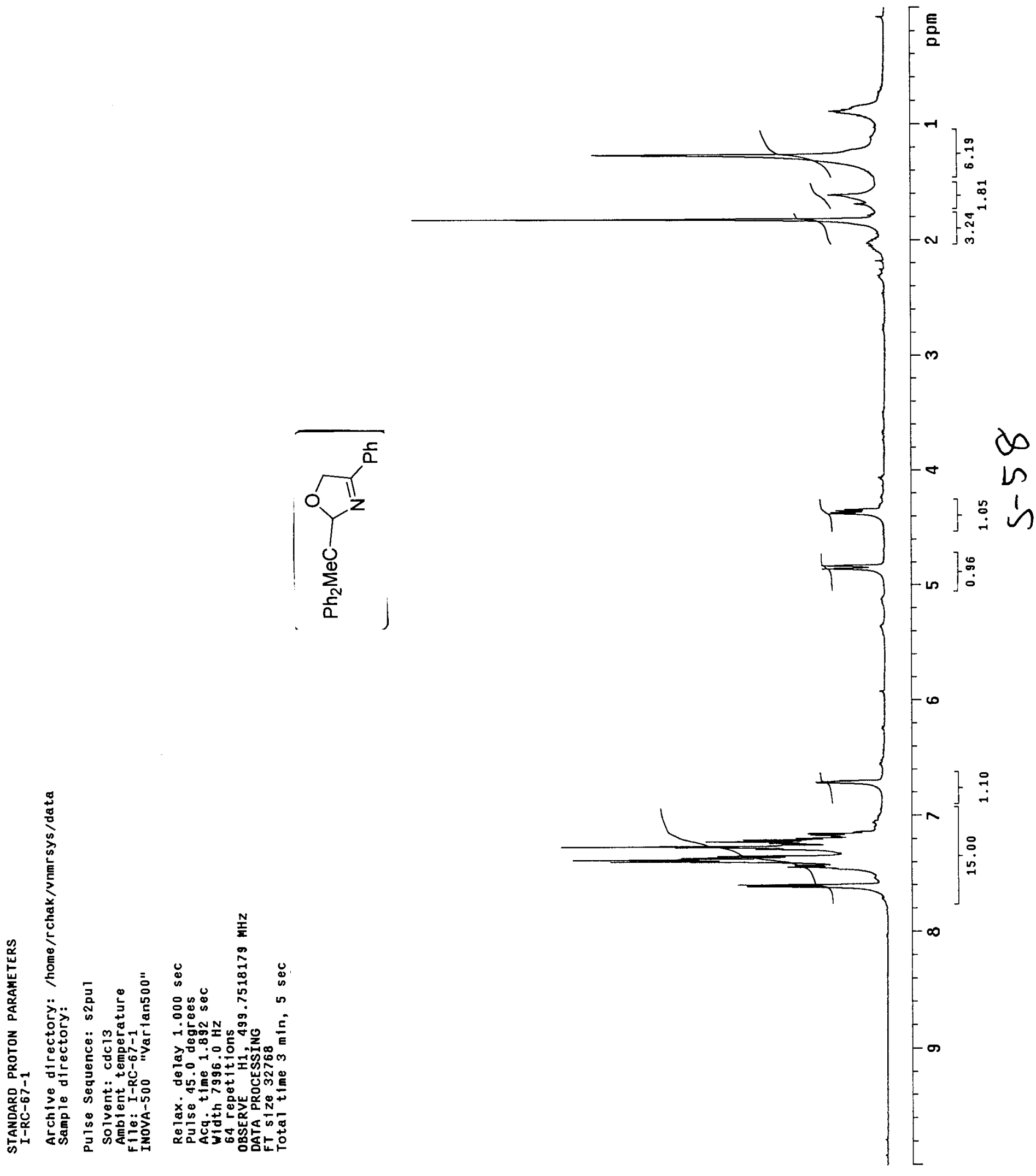




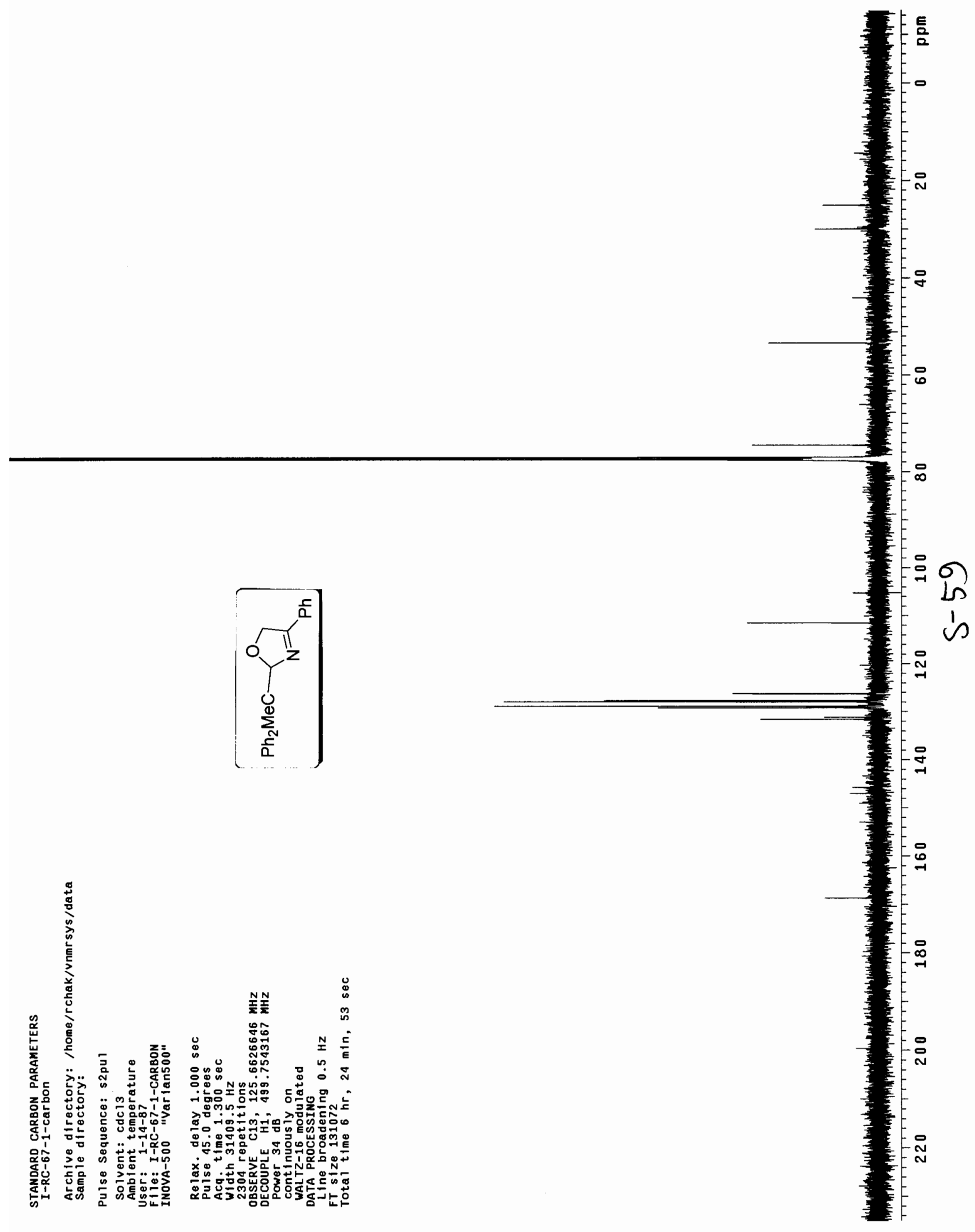




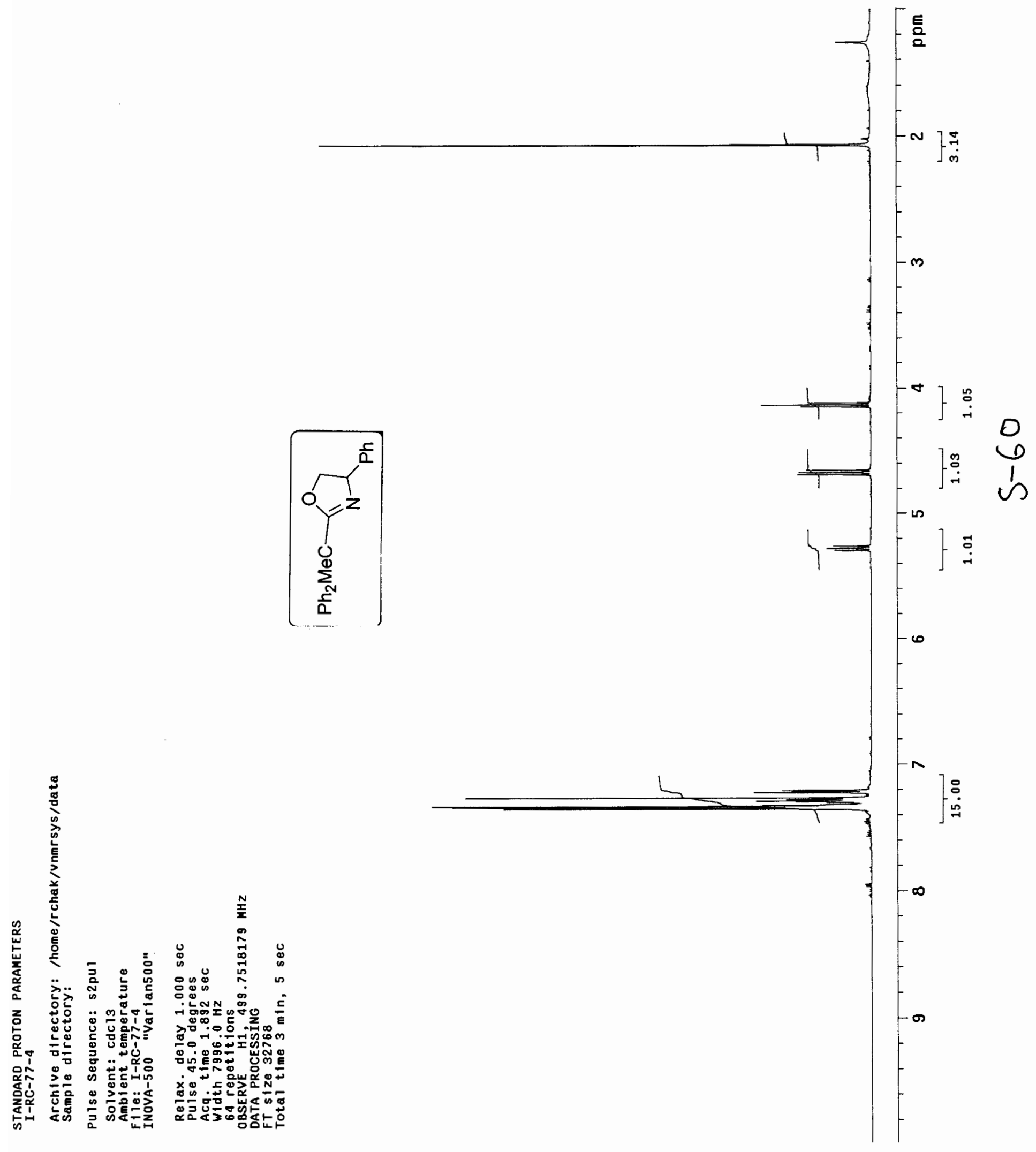




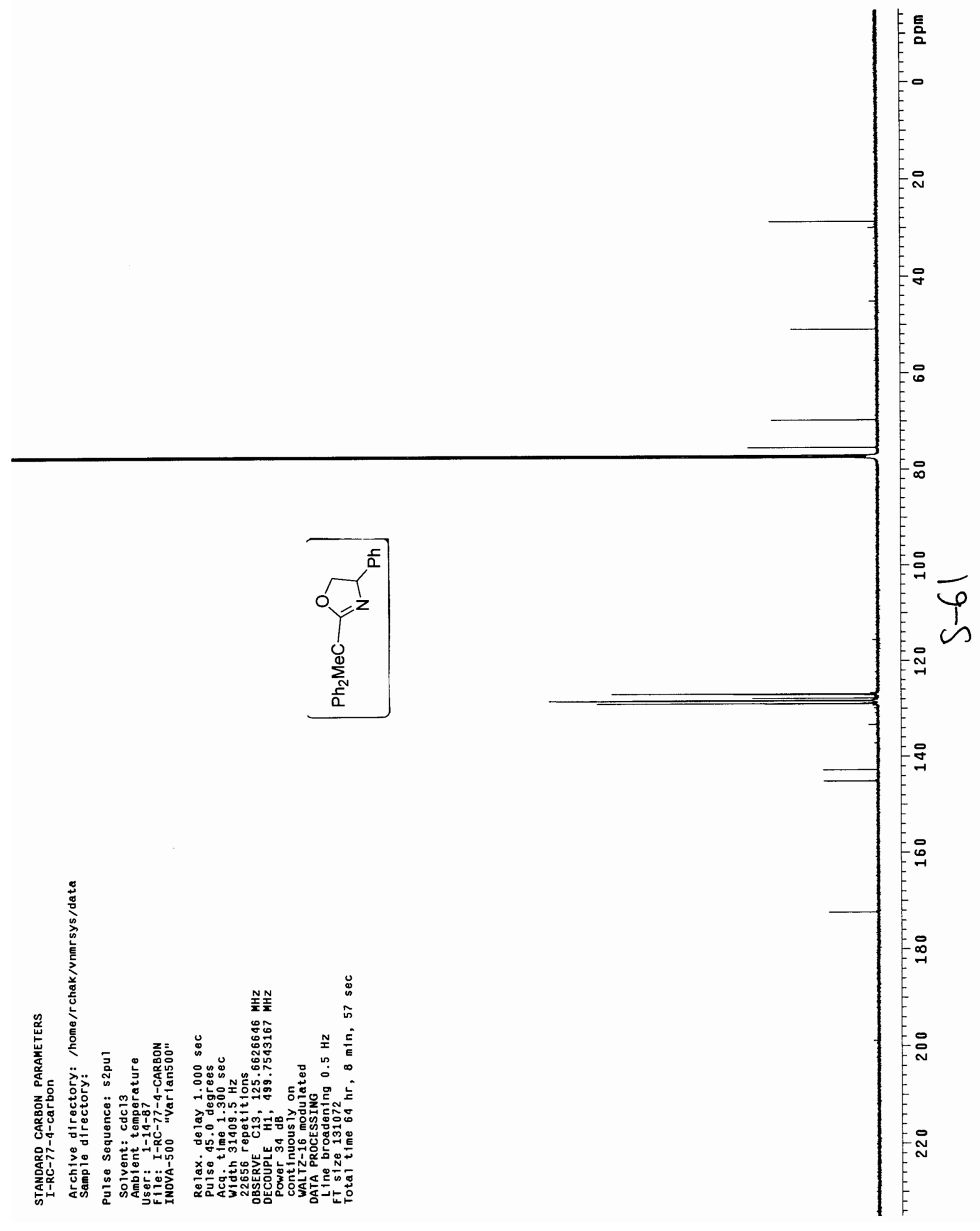



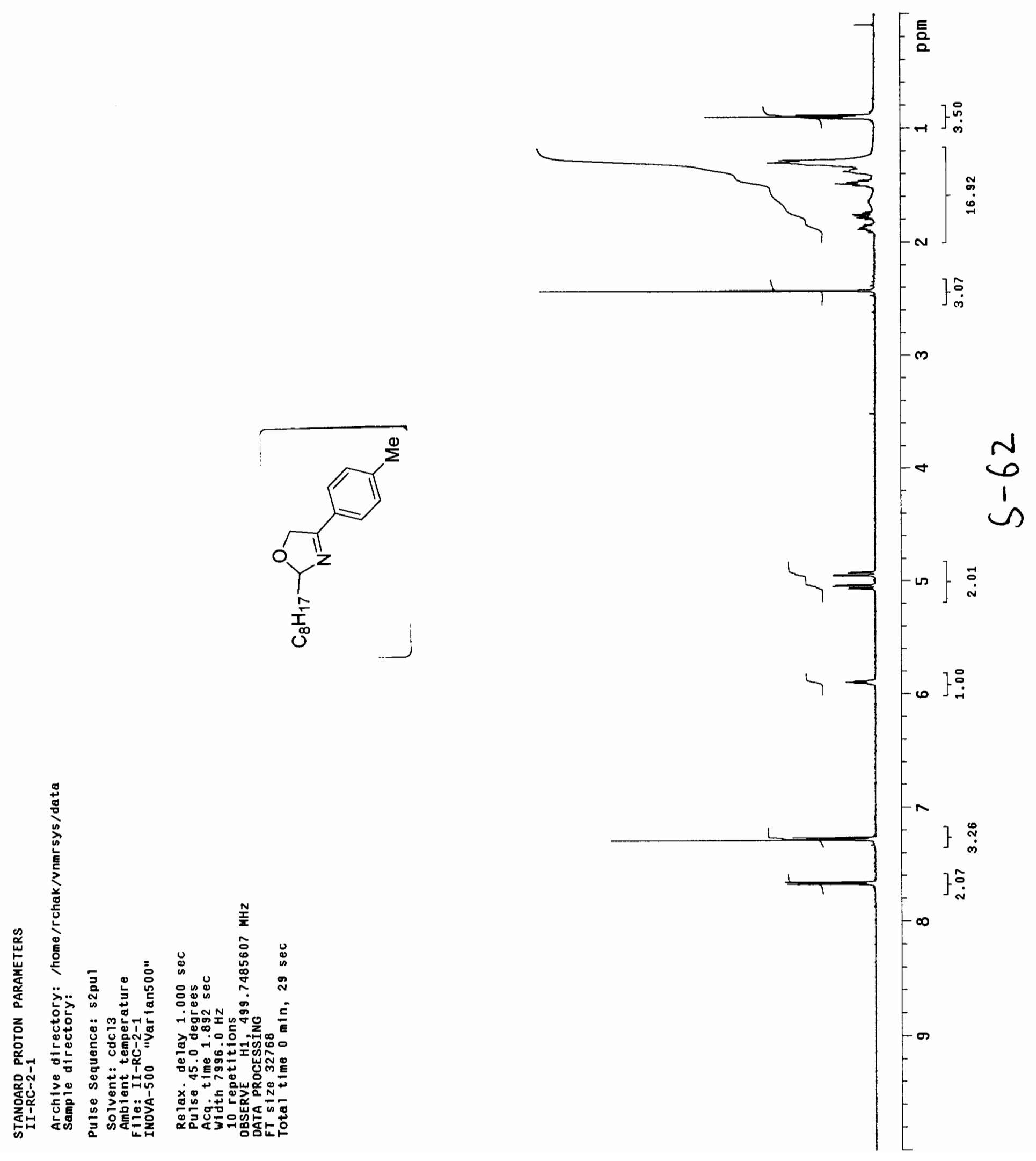


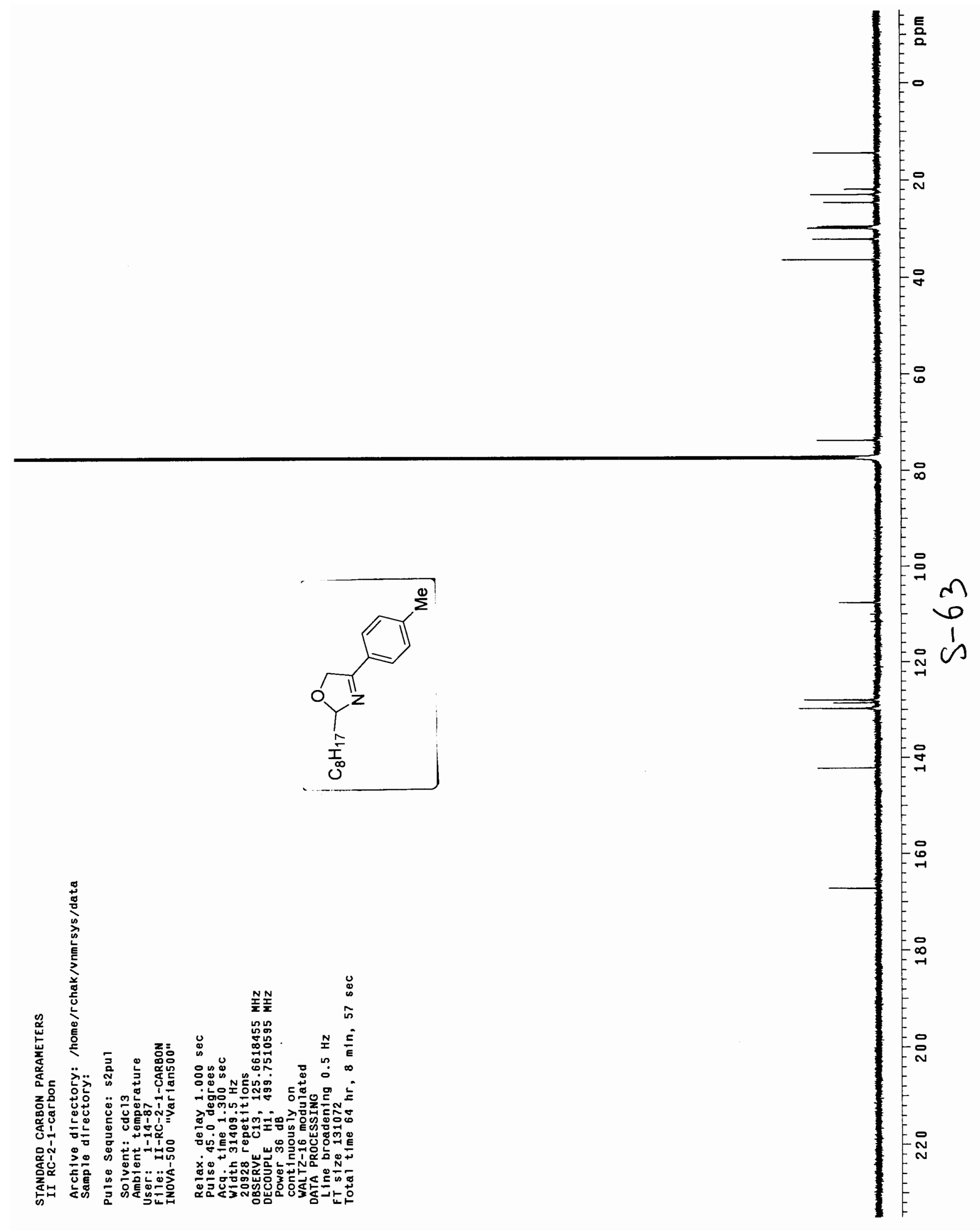



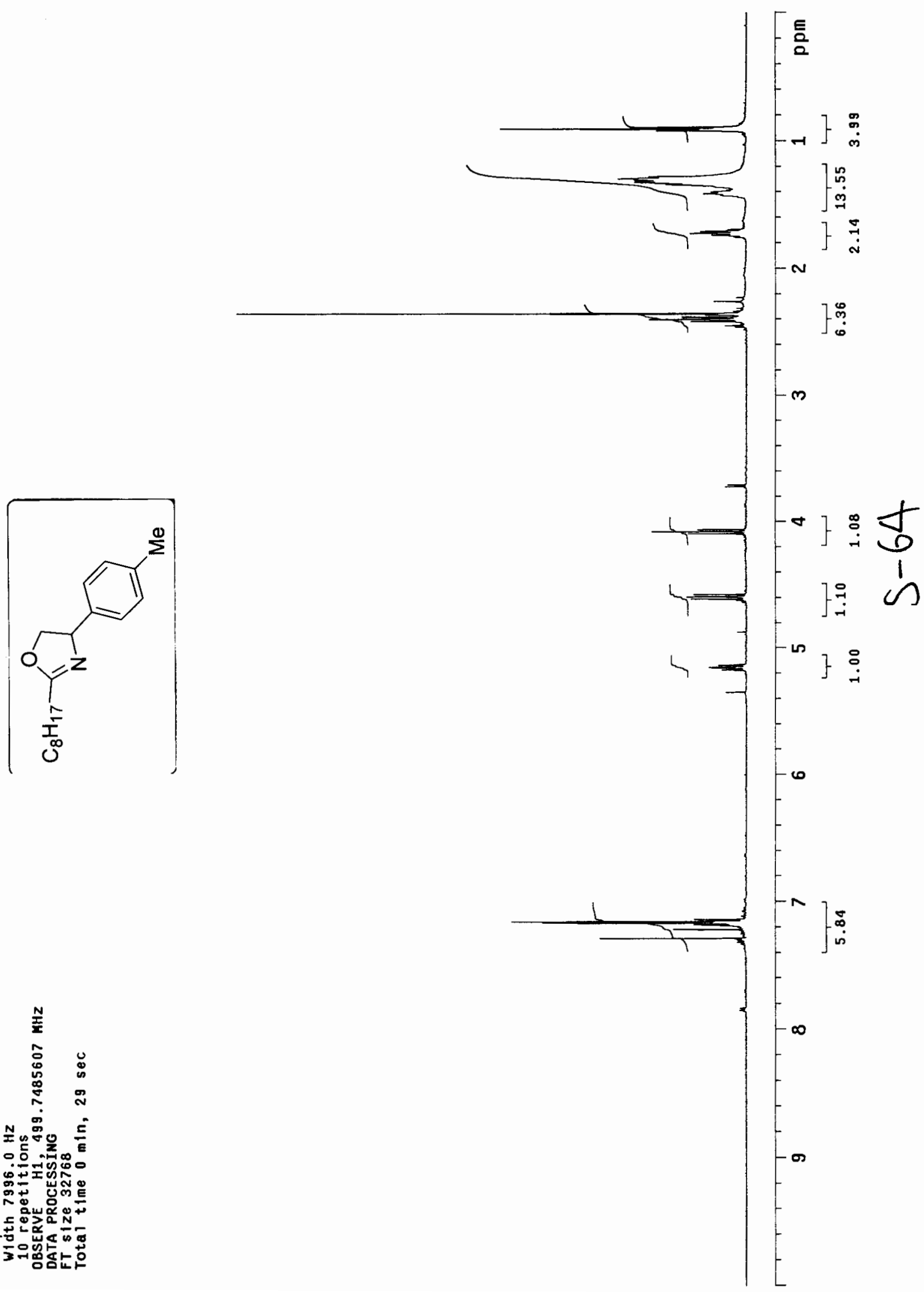


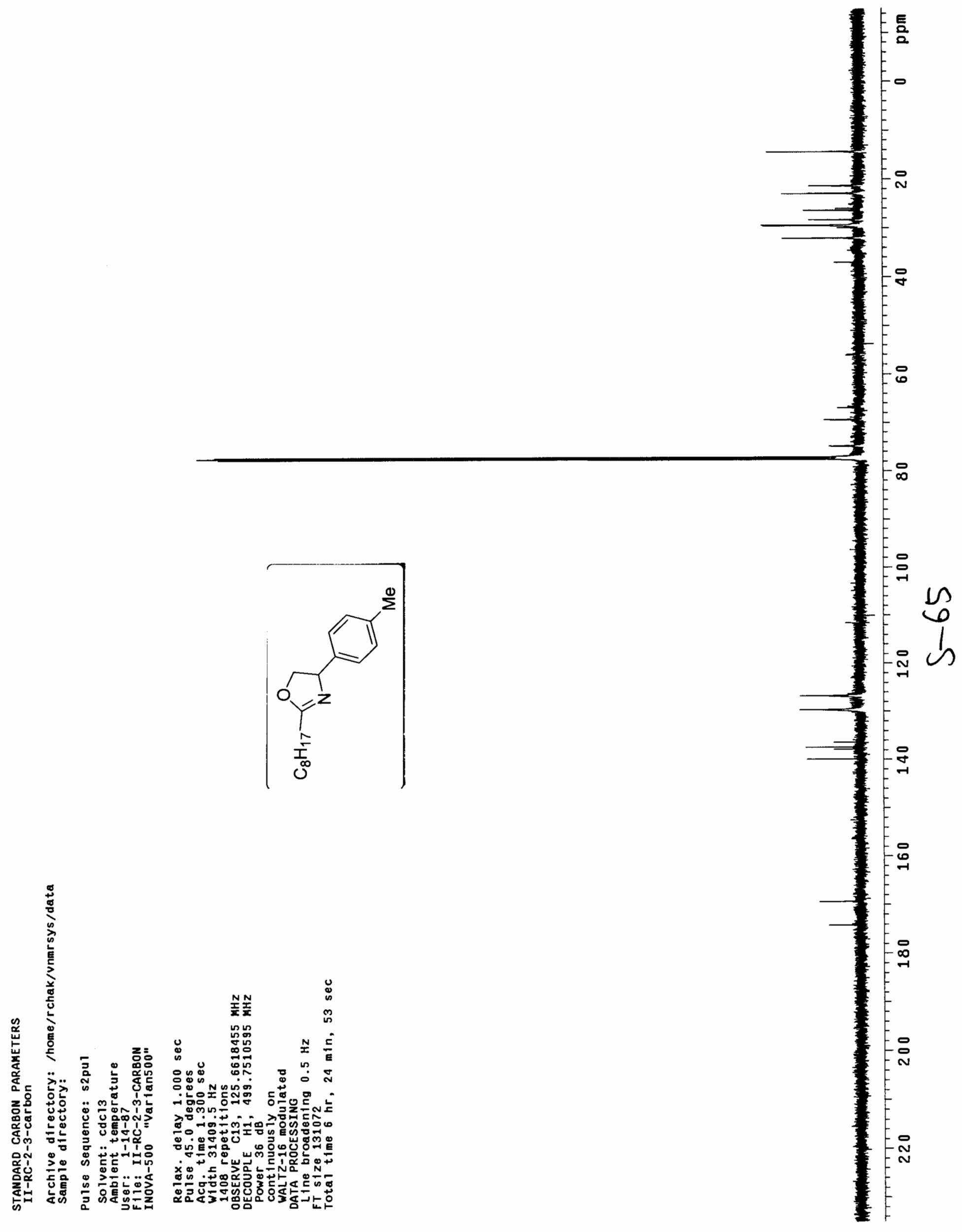




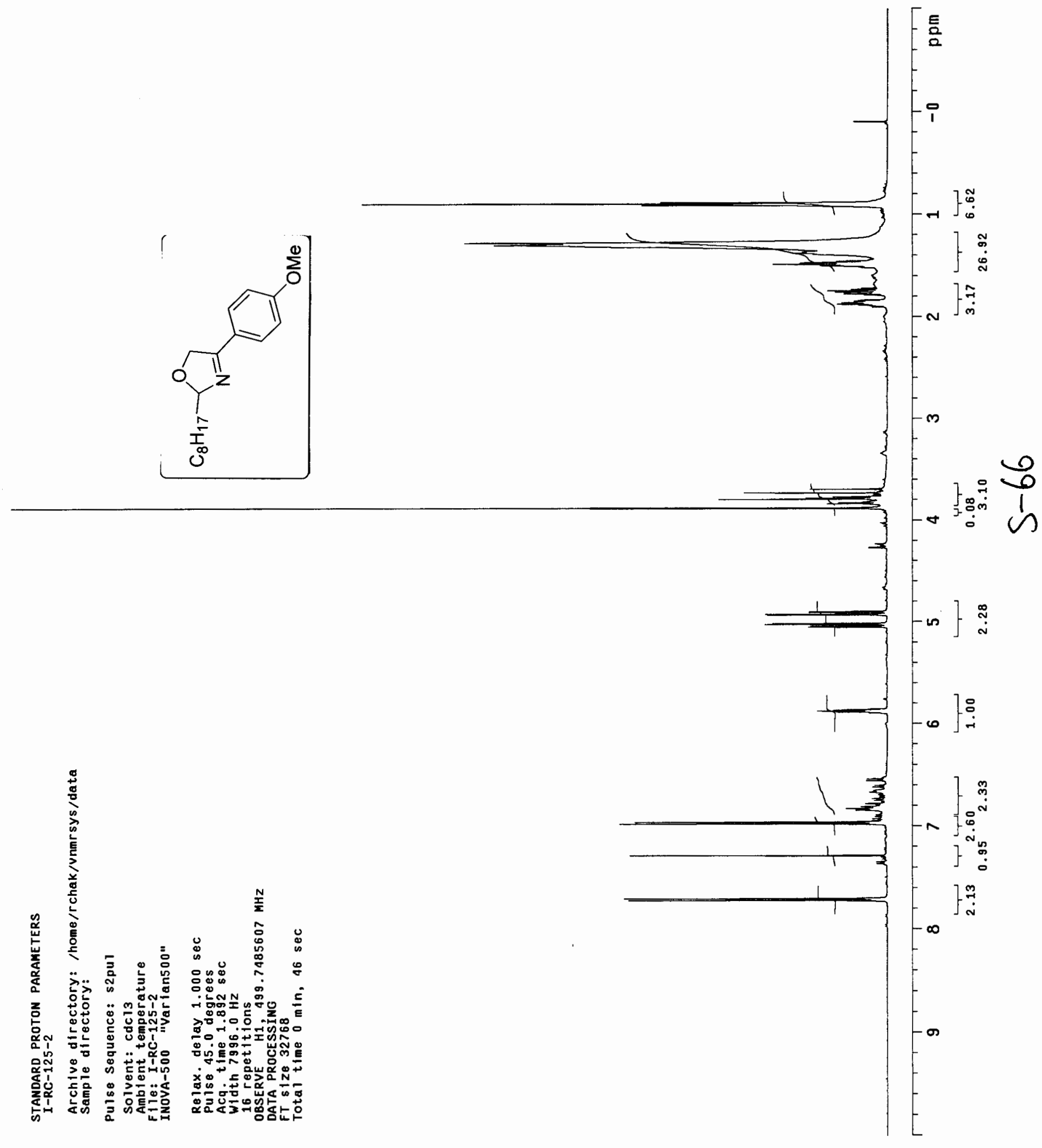




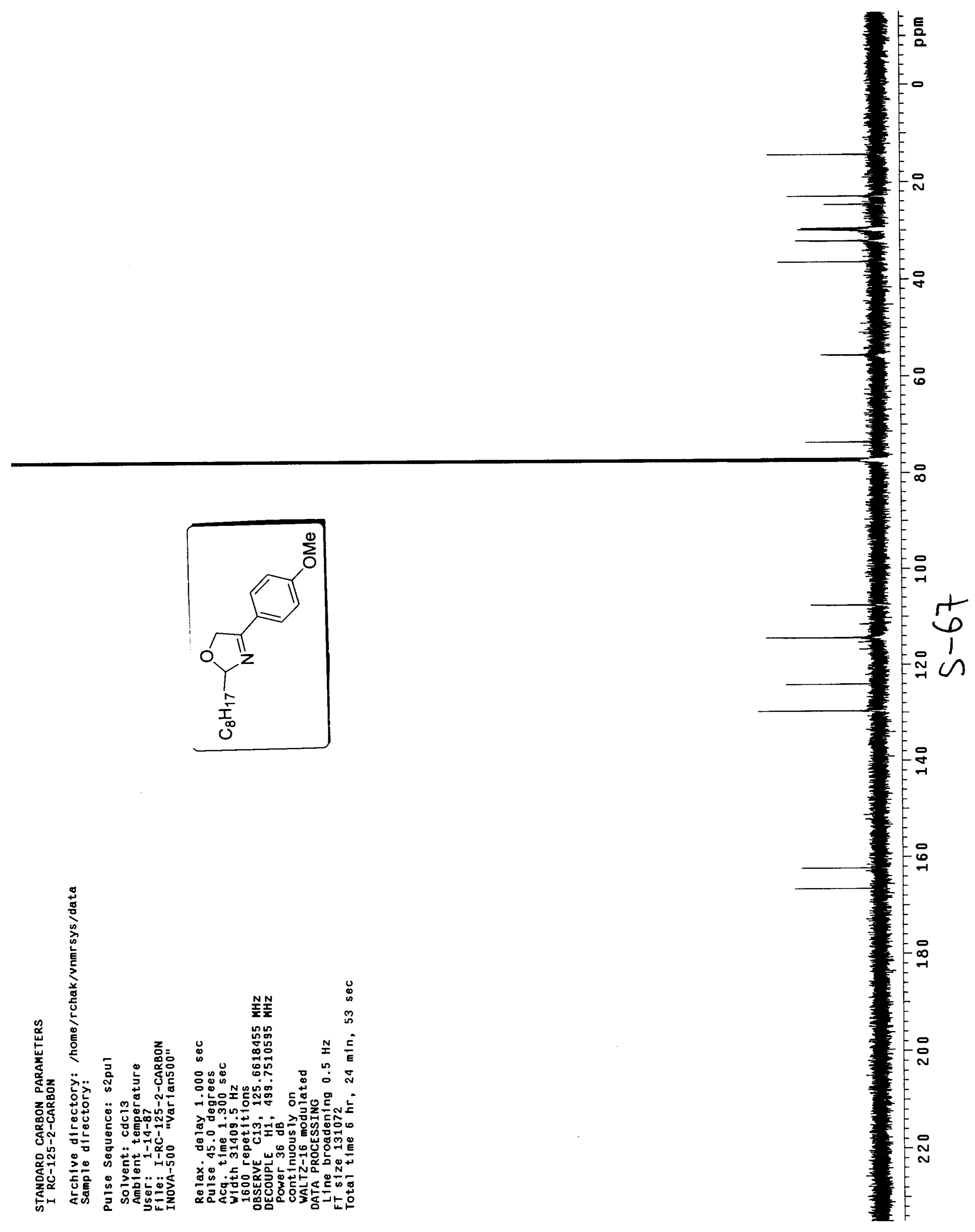



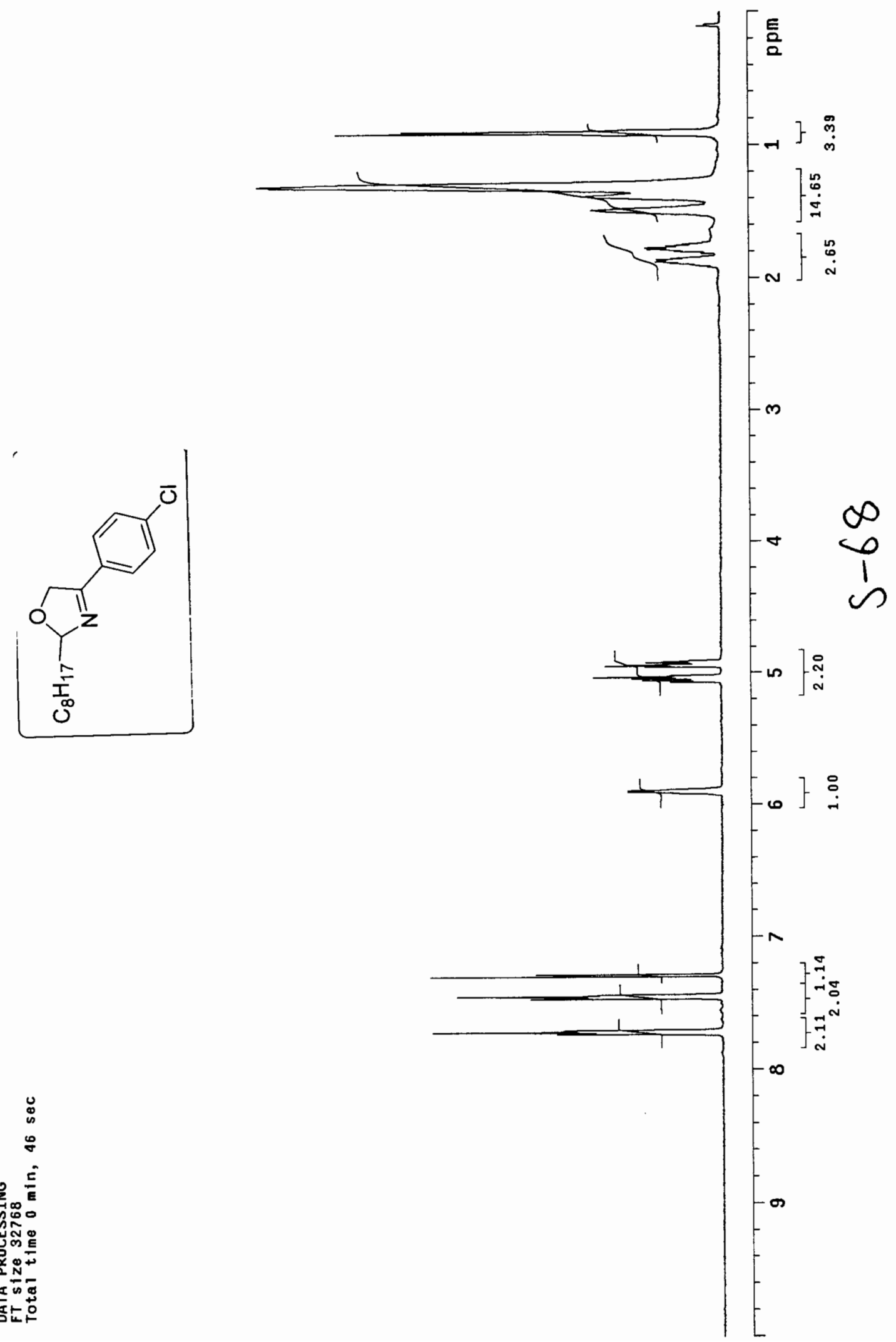


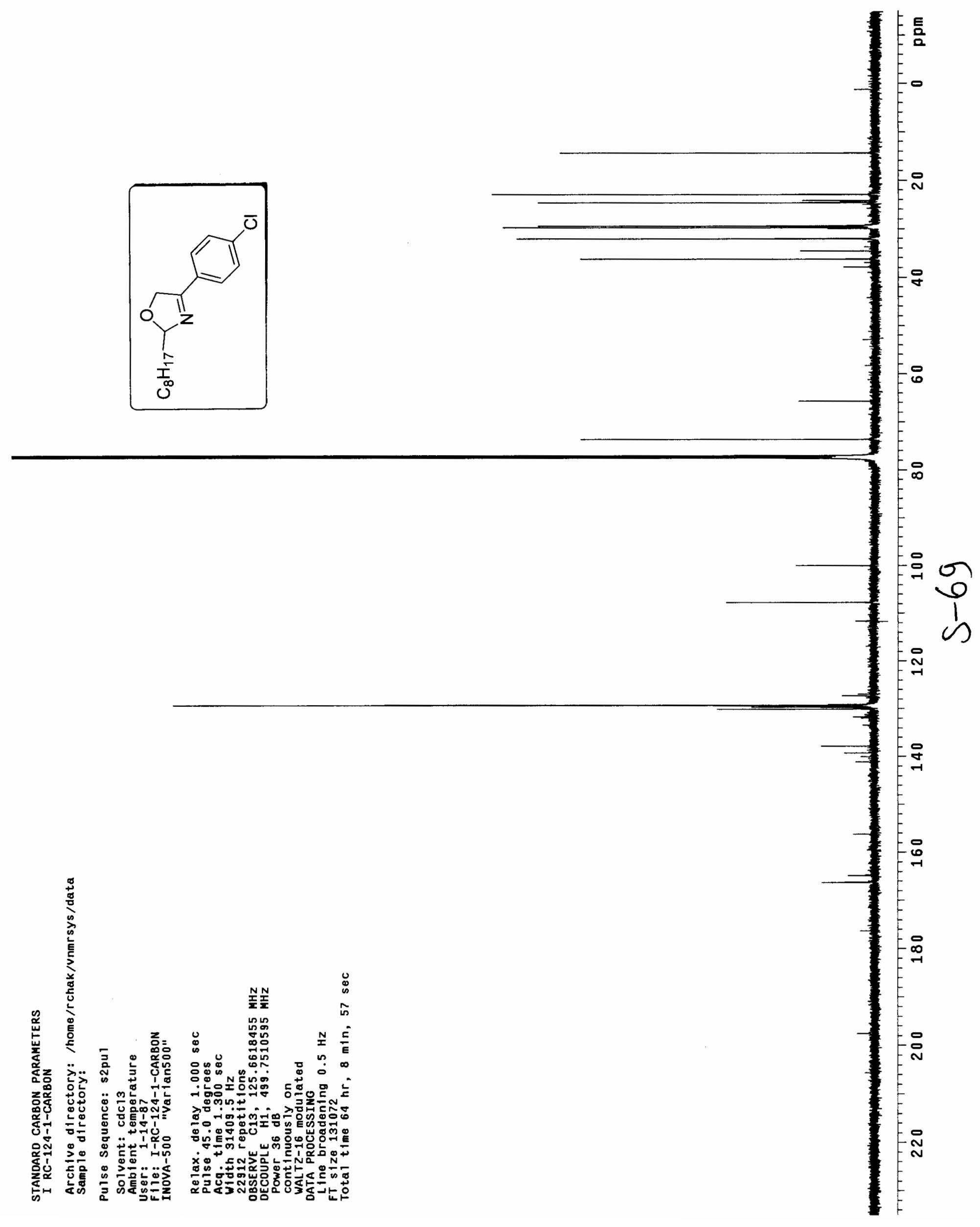



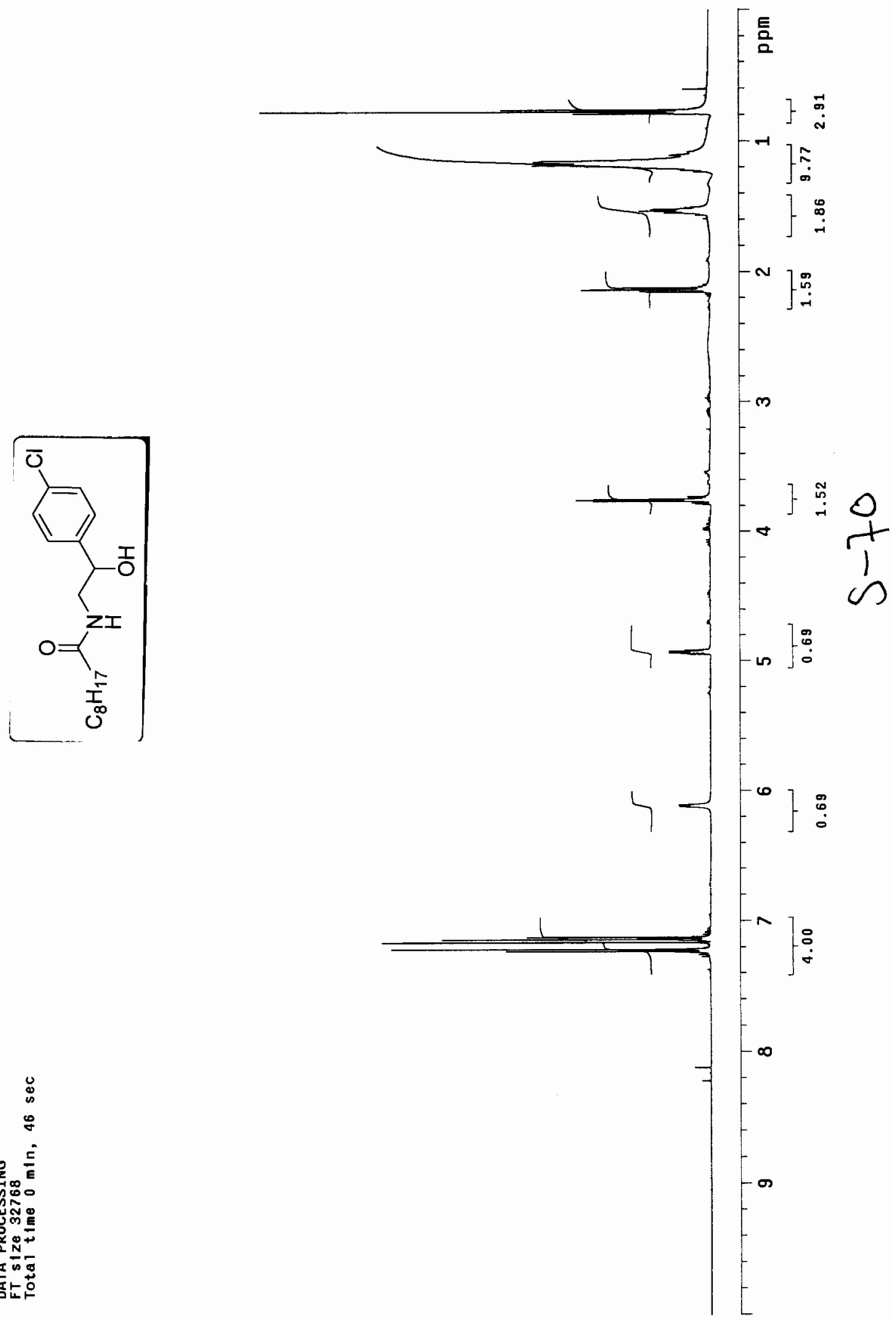


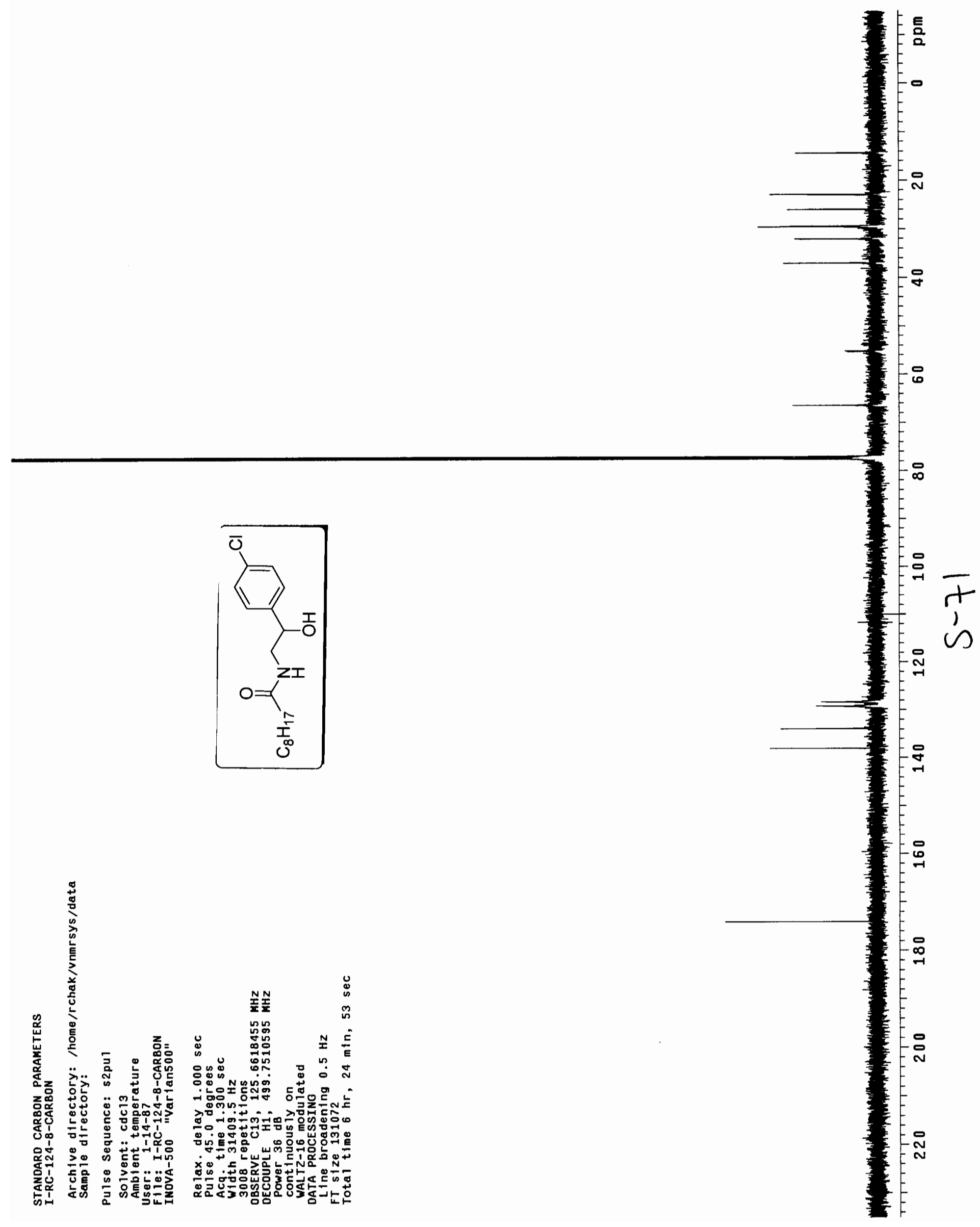




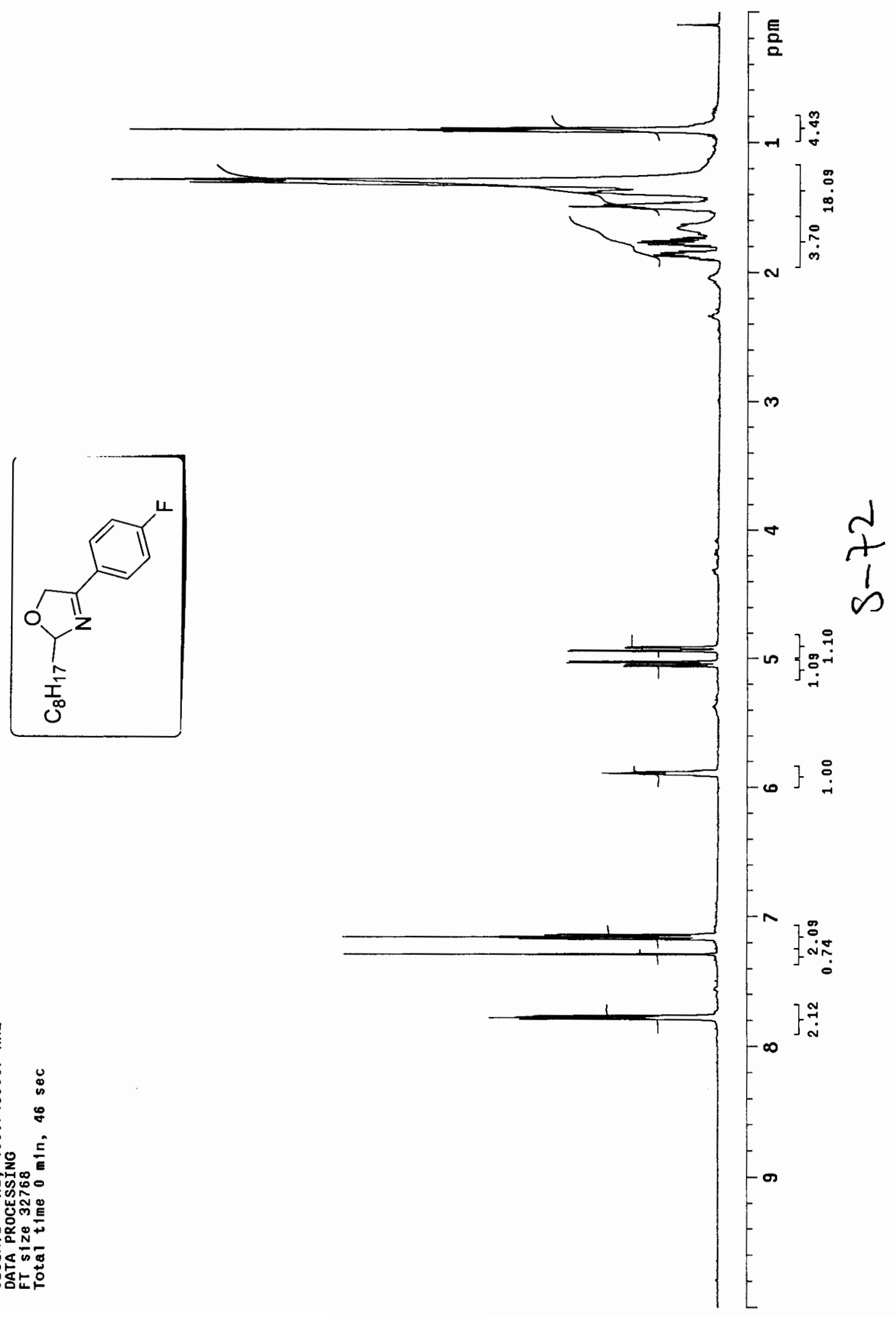




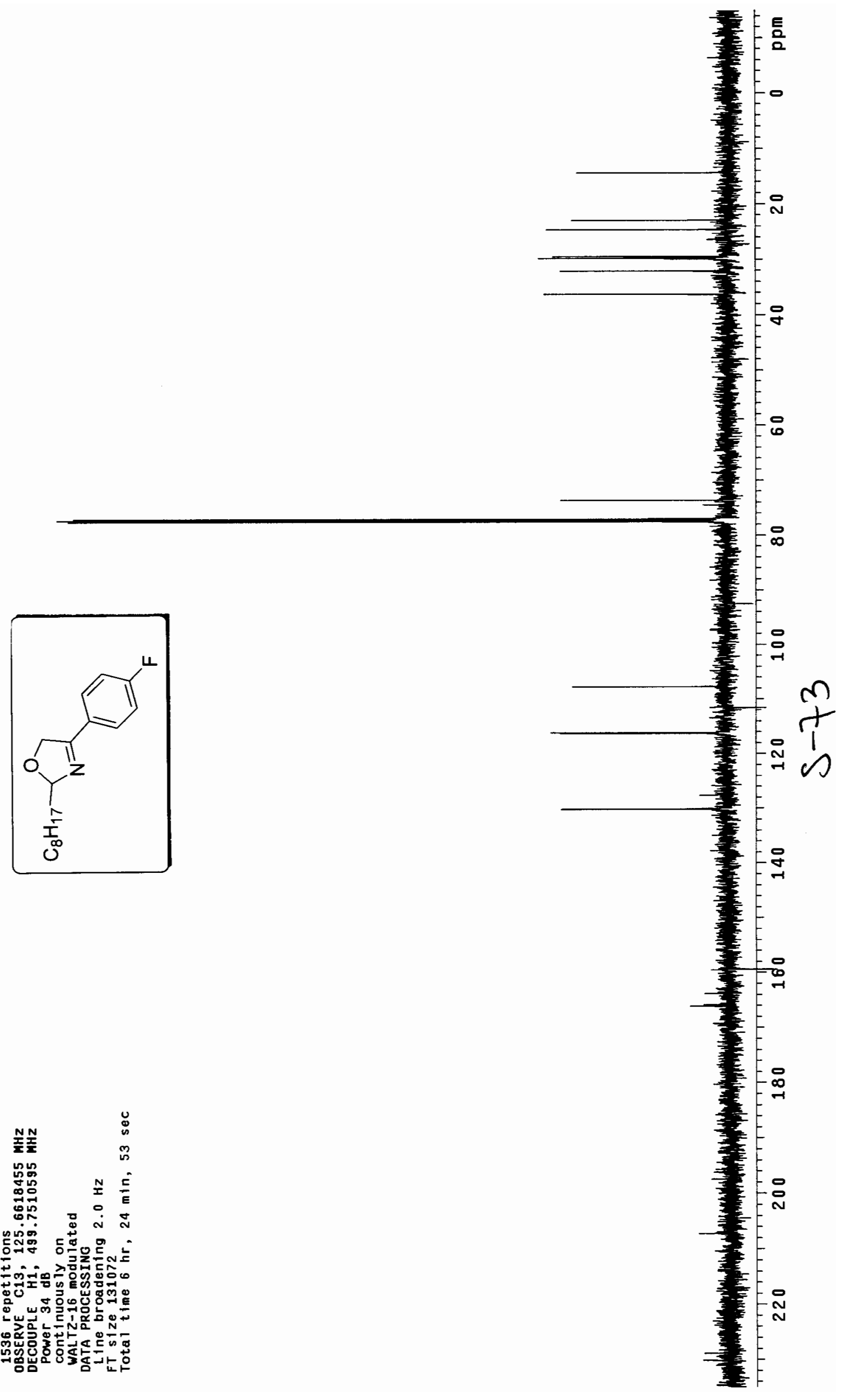




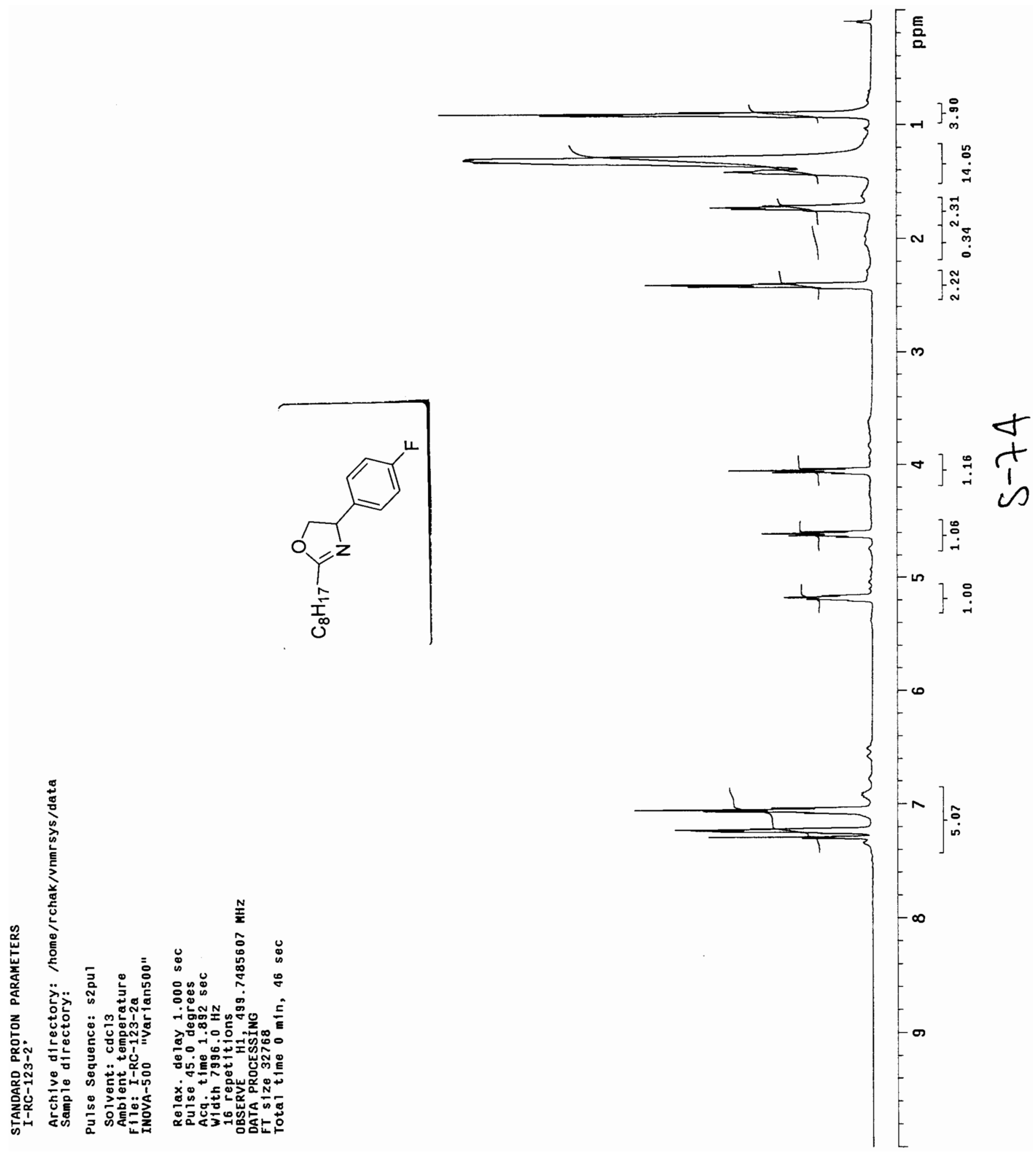




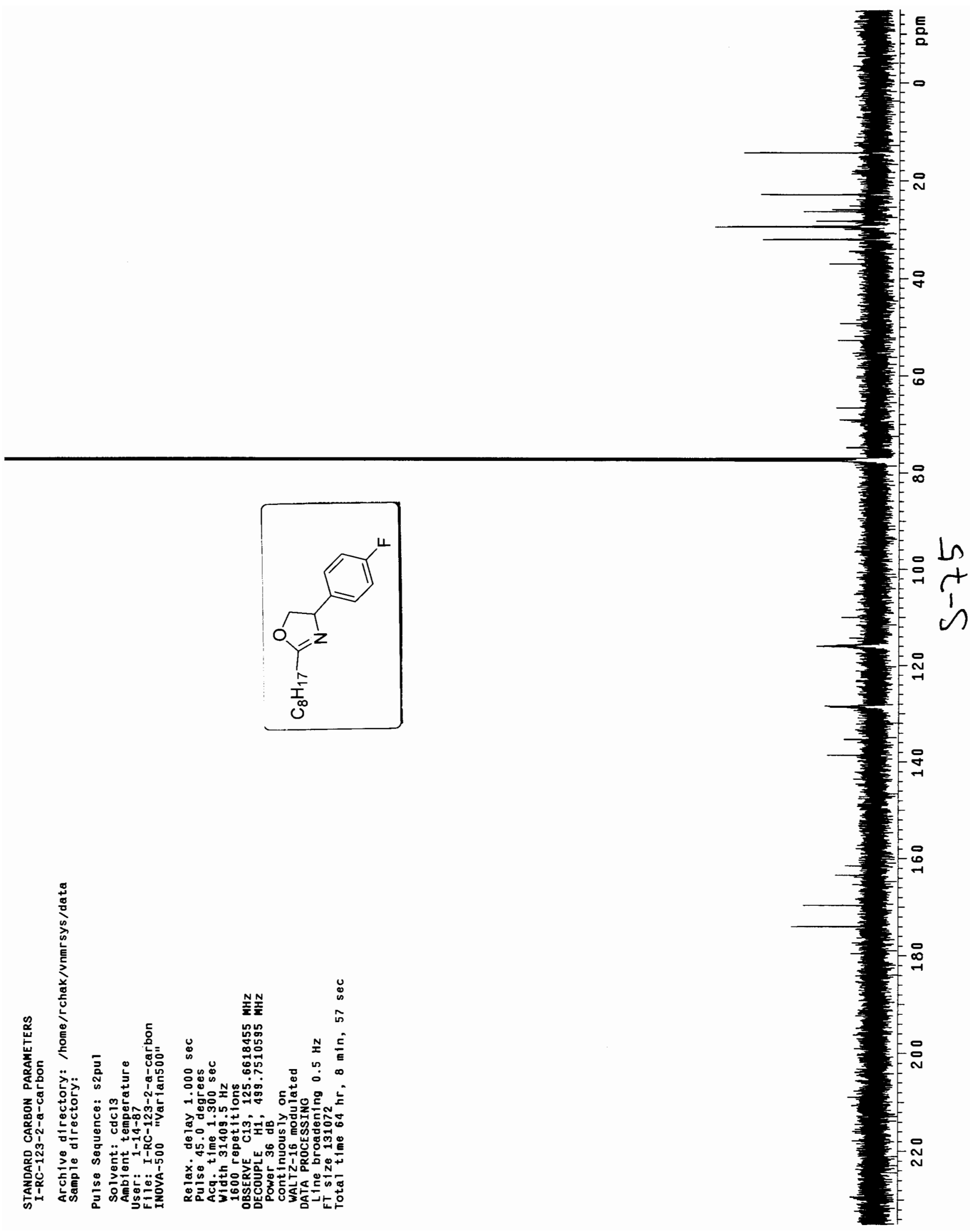




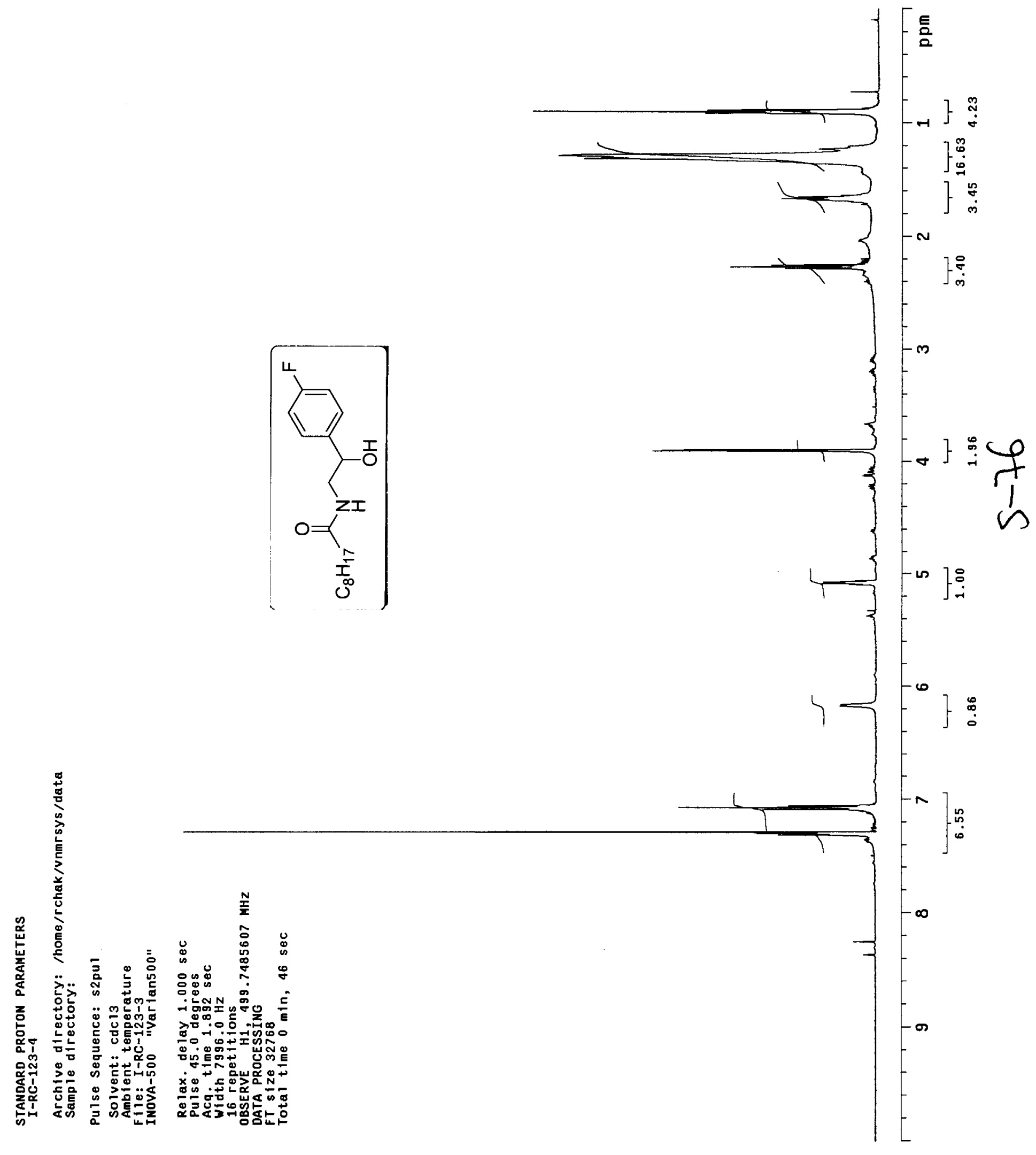




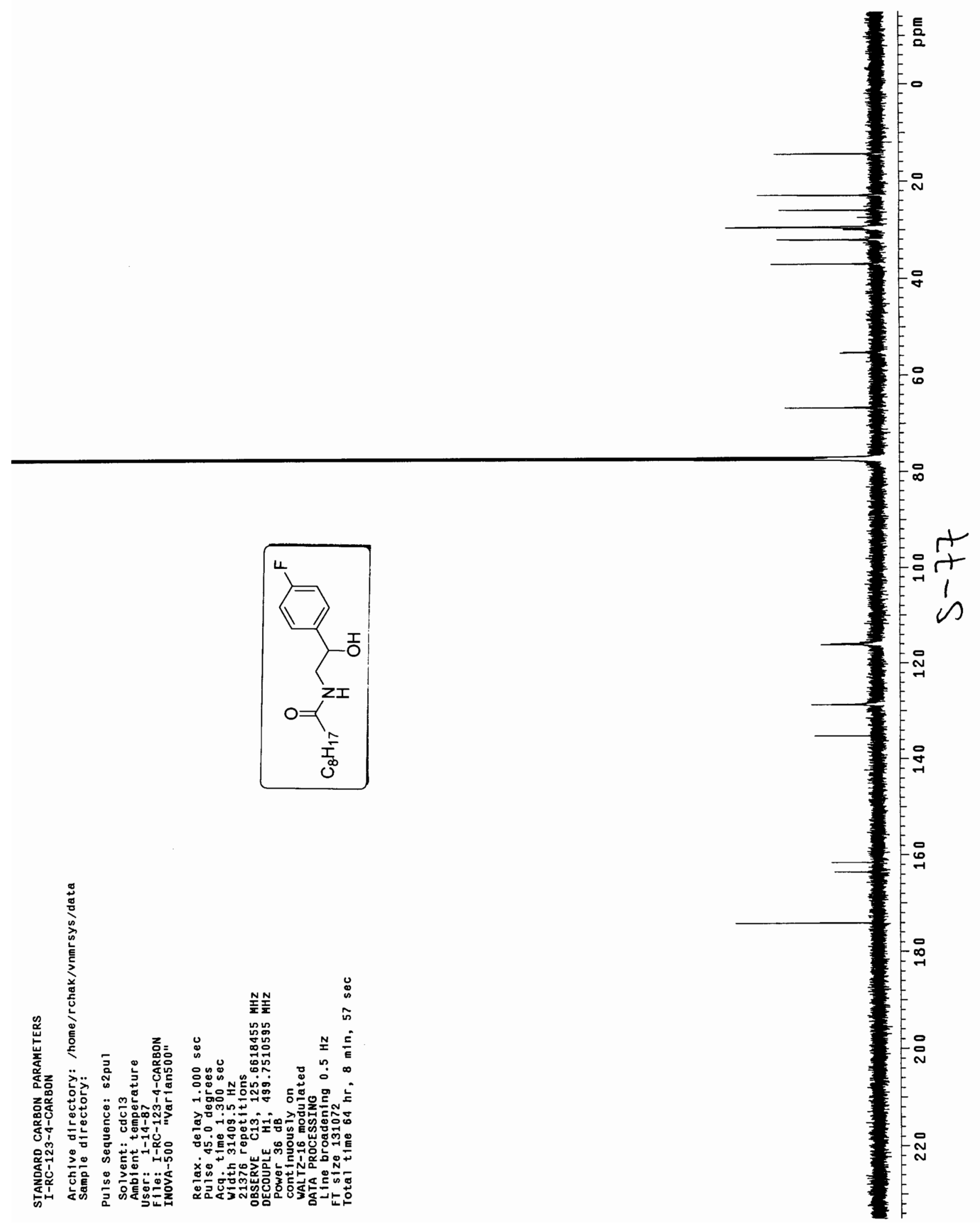




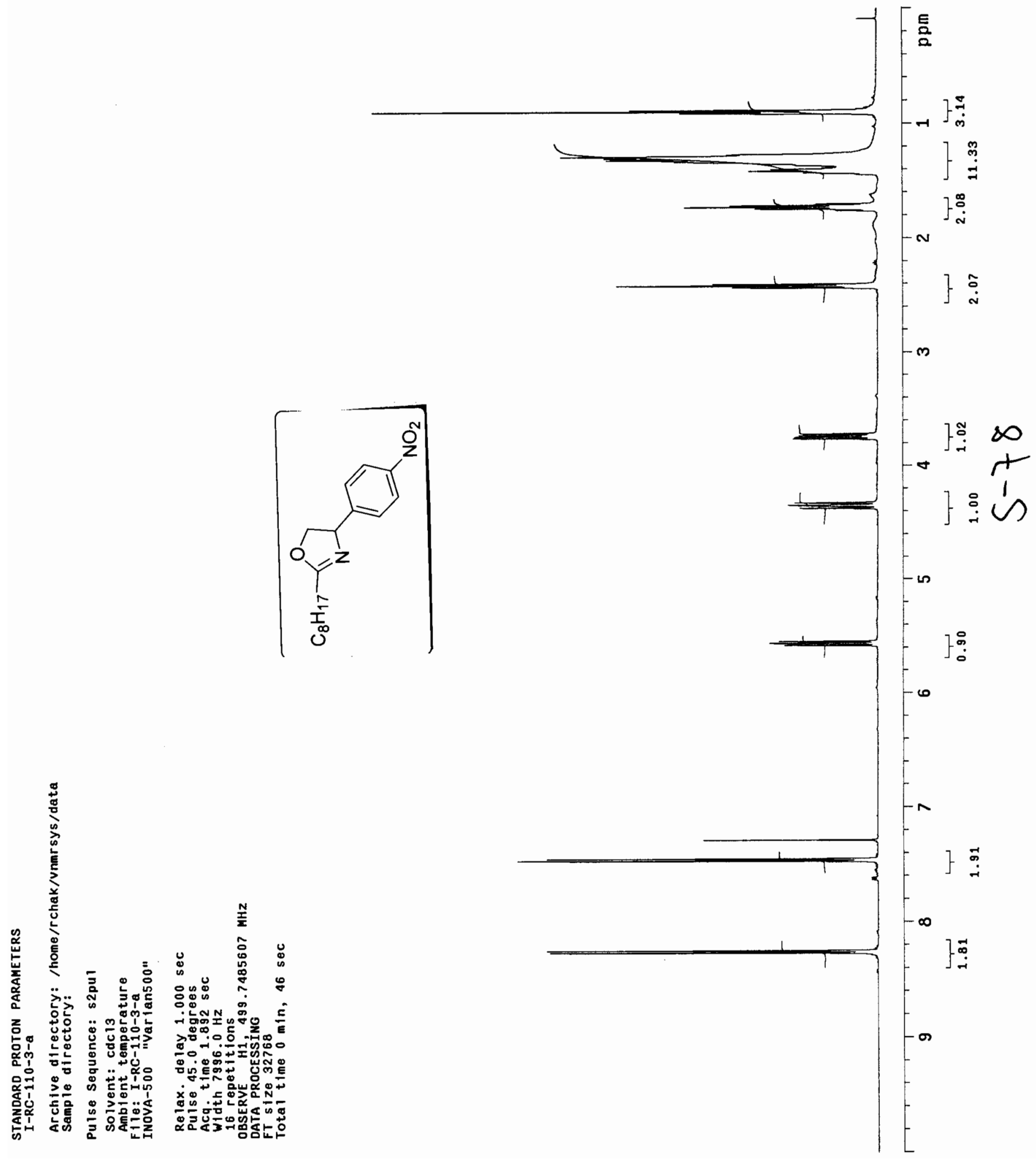



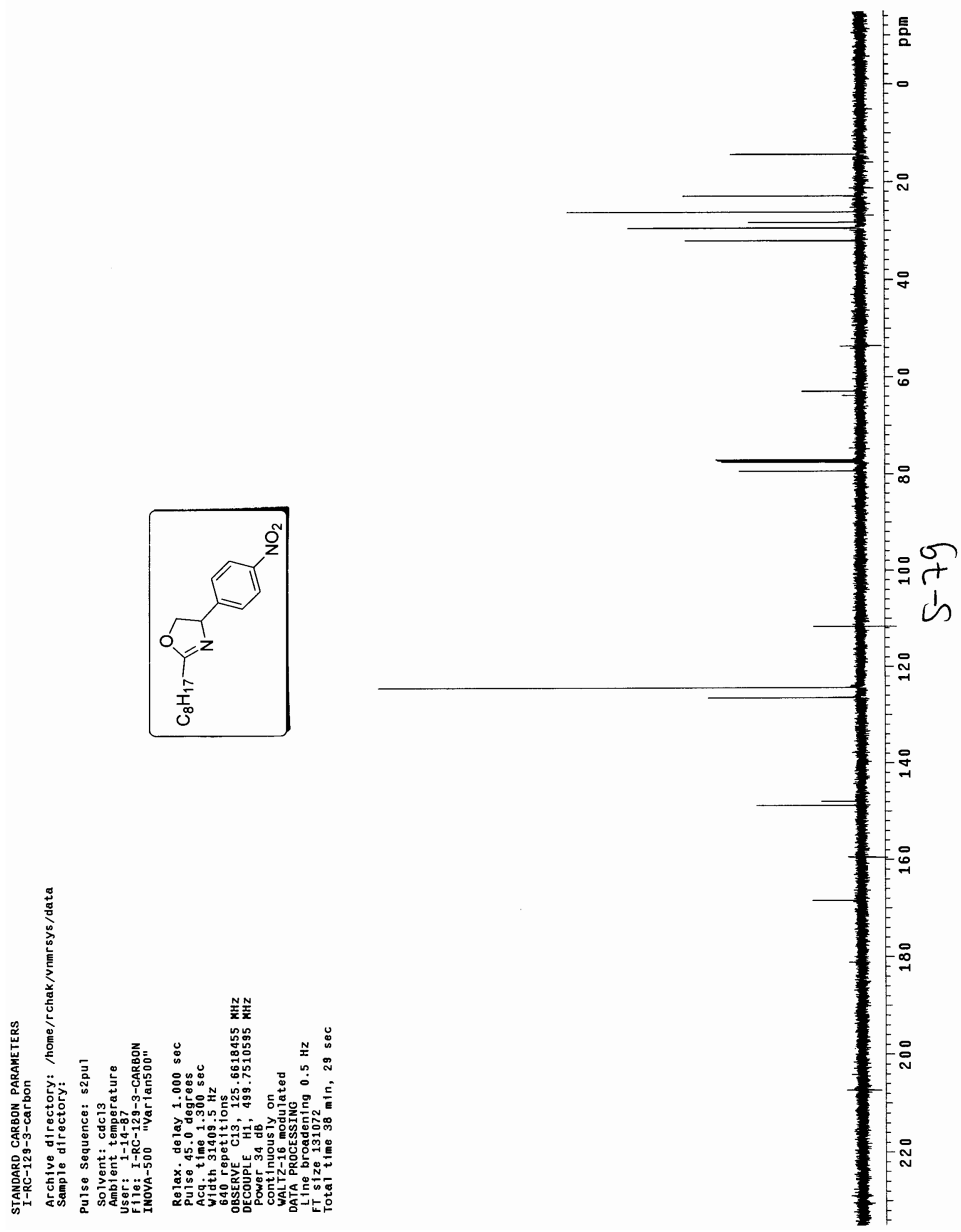


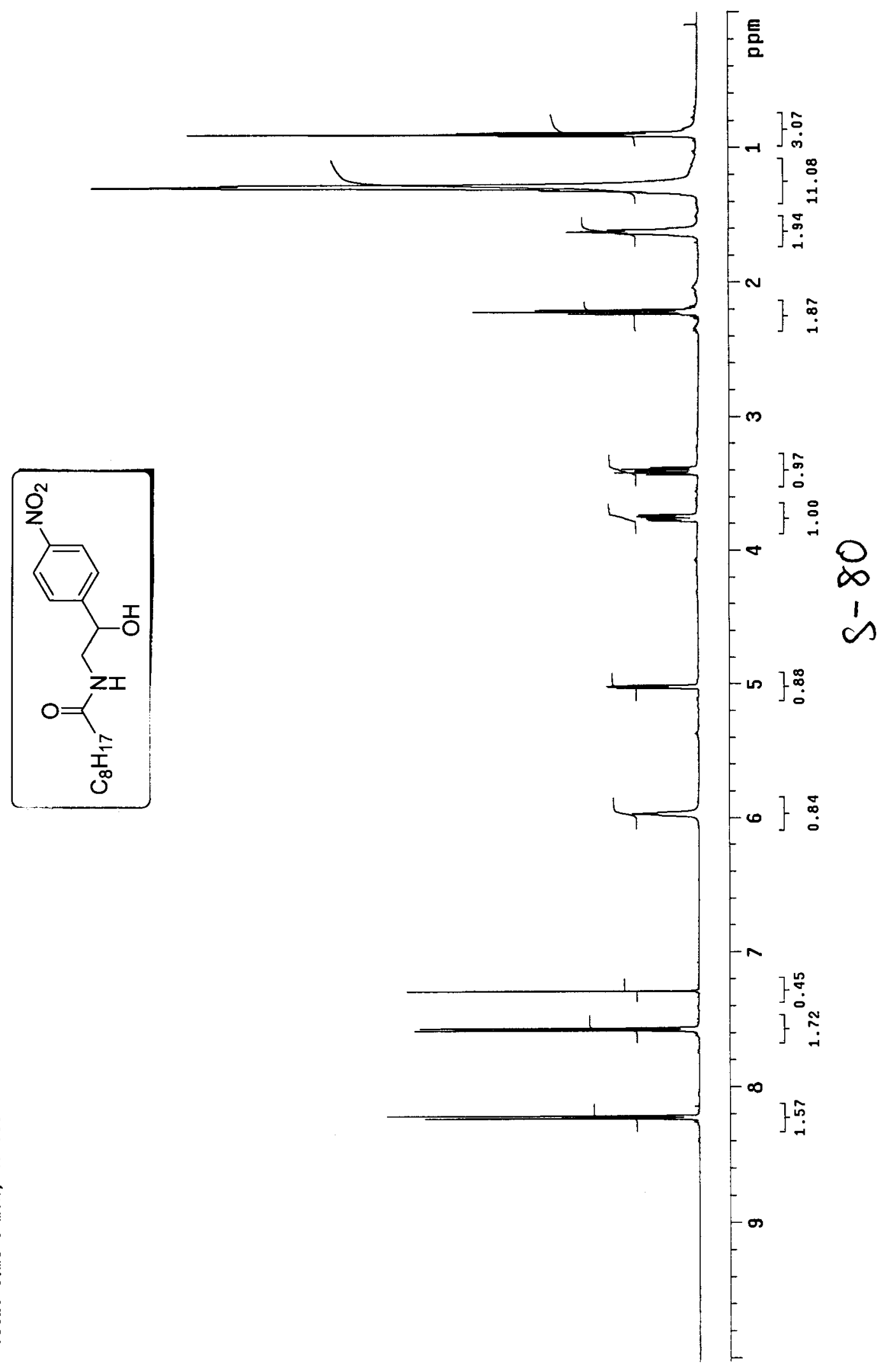




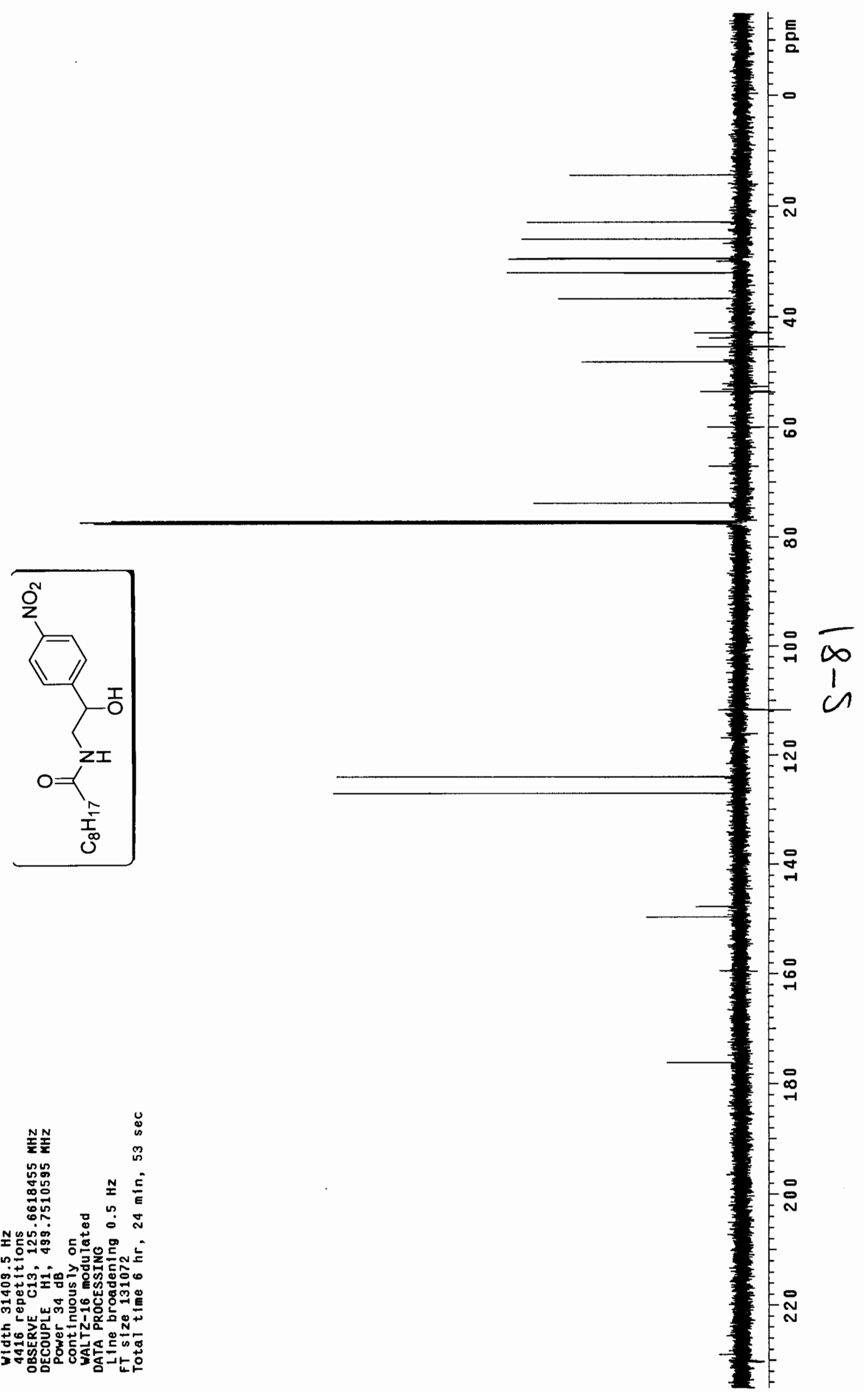




\section{Area \% Report}

Method Name: C:IEZStart|ProjectsUDefault|MethodsLAS.met

Data: $\quad$ C:IEZStart|ProjectsLDefault|DataU-RC-128-1-D

User: $\quad$ System

Acquired: $\quad$ 4/15/2005 12:01:27 PM

Printed: $\quad$ 6/14/2005 1:34:37 PM

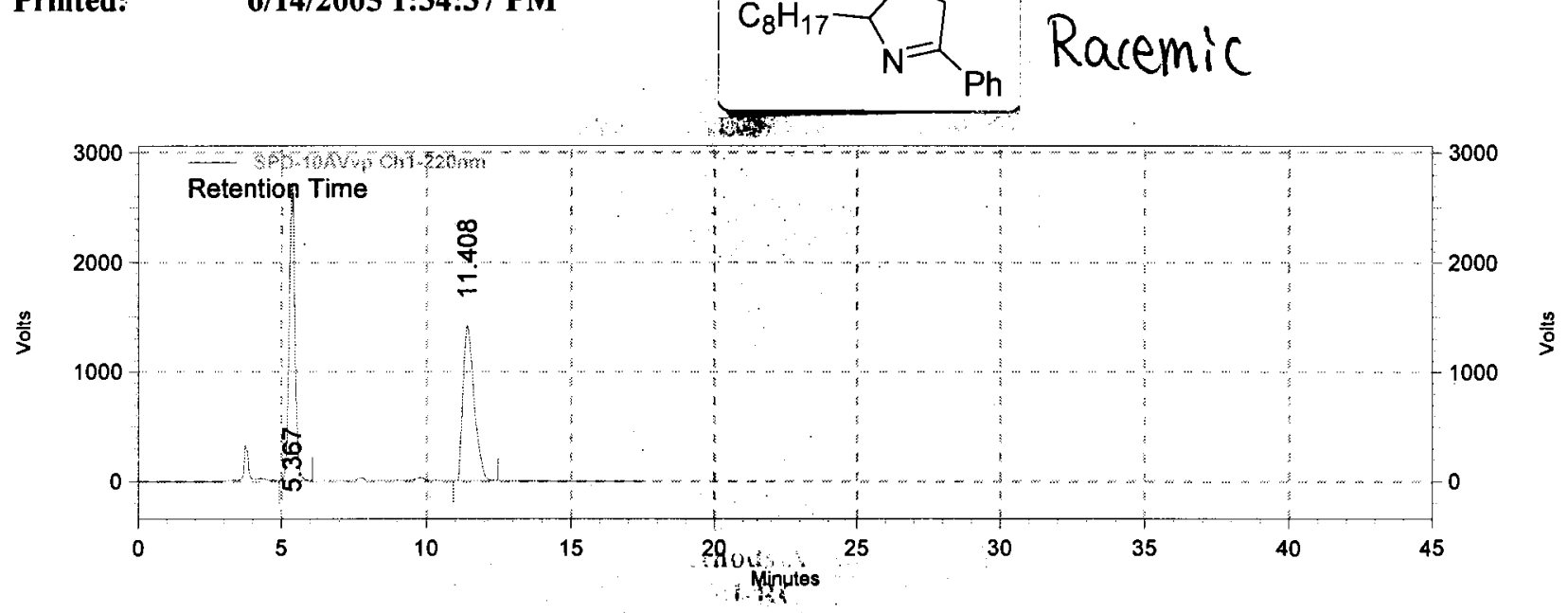

SPD-10AVvp

Results

\begin{tabular}{|c|c|c|c|c|}
\hline Retention Time & Area & Area \% & Height & Height $\%$ \\
\hline 5.367 & 37525895 & 49.79 & 2714168 & 65.75 \\
\hline 11.408 & 37837558 & 50.21 & 1414100 & 34.25 \\
\hline Totals & 75363453 & 100.00 & 4128268 & 100.00 \\
\hline
\end{tabular}

$S-82$ 


\section{Area \% Report}

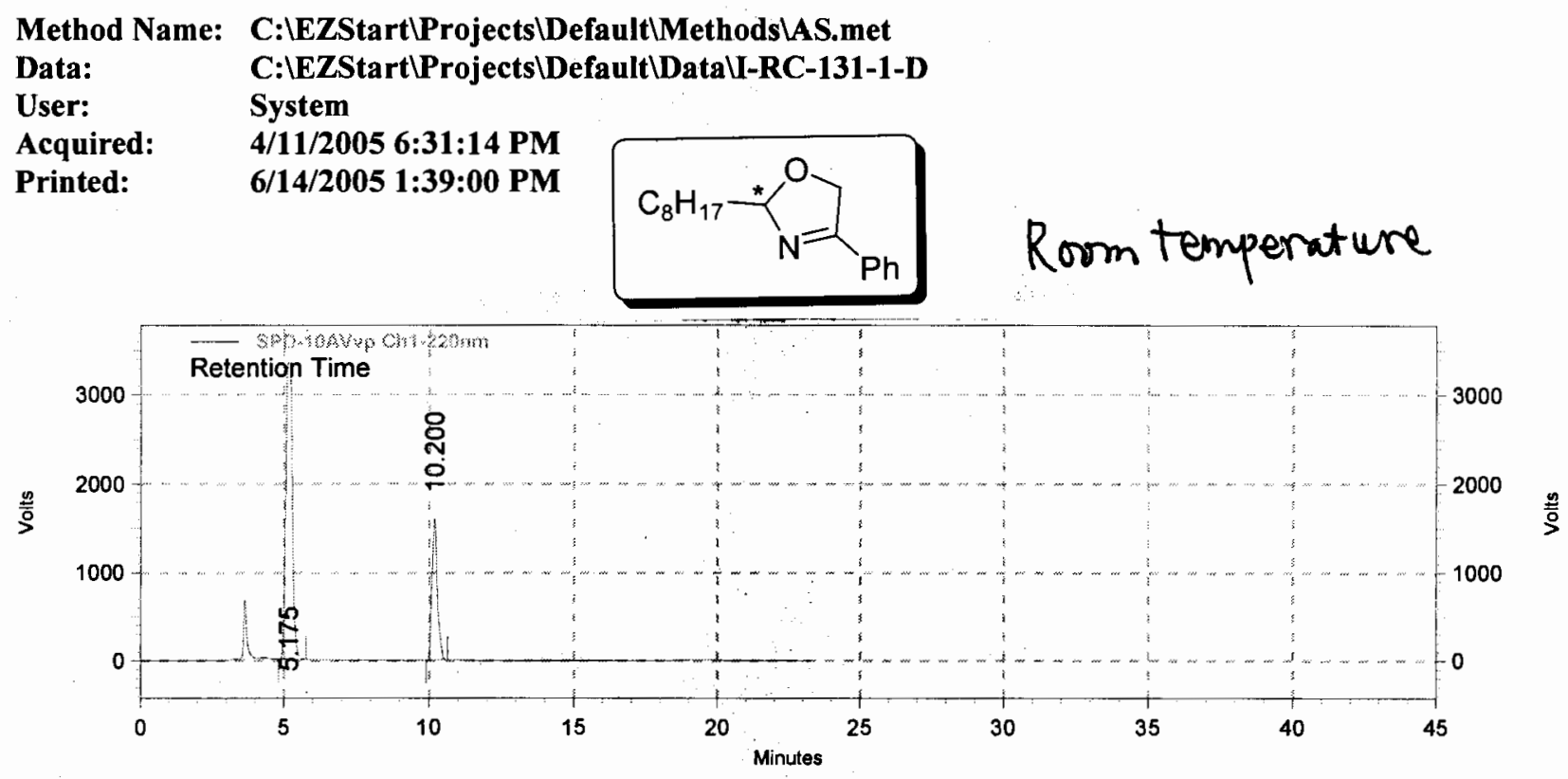

SPD-10AVvp

Ch1-220nm

Results

\begin{tabular}{r|rrrrr|} 
Retention Time & Area & Area \% & Height & Height \% \\
\hline 5.175 & 53741266 & 72.28 & 3347989 & 67.65 \\
10.200 & 20605220 & 27.72 & 1601254 & 32.35 \\
& & & & \\
\hline Totals & 74346486 & 100.00 & 4949243 & 100.00 \\
\hline
\end{tabular}

$5-83$ 


\section{Area \% Report}

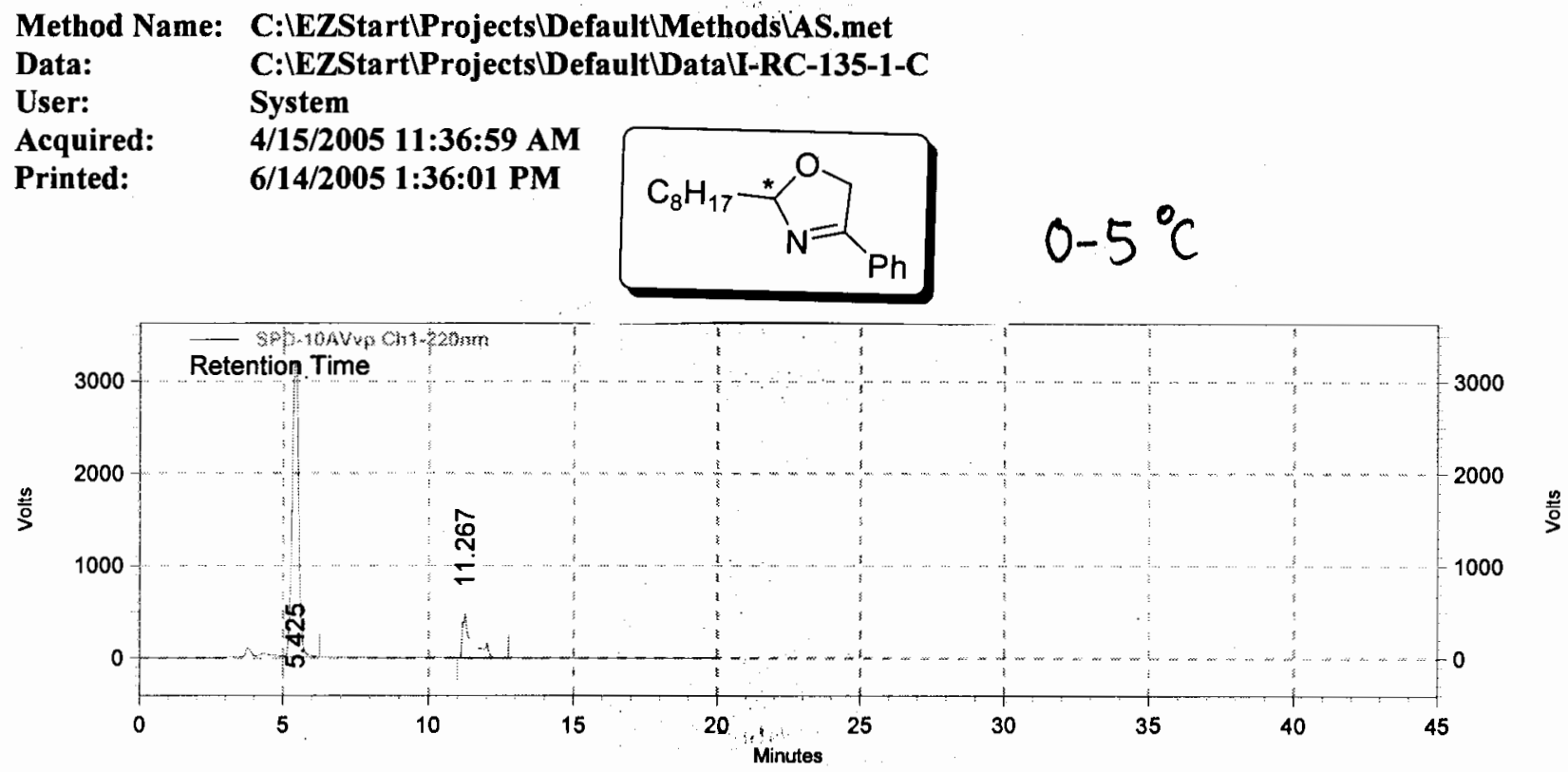

\section{SPD-10AVvp}

Ch1-220nm

Results

Retention Time

5.425

11.267

Area

Area \%

$50599490 \quad 82.39 \quad 3214572$

17.61

Height

Height \%

87.21

10813433

471645

12.79

Totals 


\section{Area \% Report}

Method Name: C:IEZStart|Projects\Default $\backslash$ Methods\AS.met

Data: C: $\quad$ IEZStart|ProjectsUDefault|DatalI-RC-142-1

User: System

Acquired: $\quad$ 4/21/2005 3:34:09 PM

Printed: $\quad$ 6/14/2005 1:41:04 PM
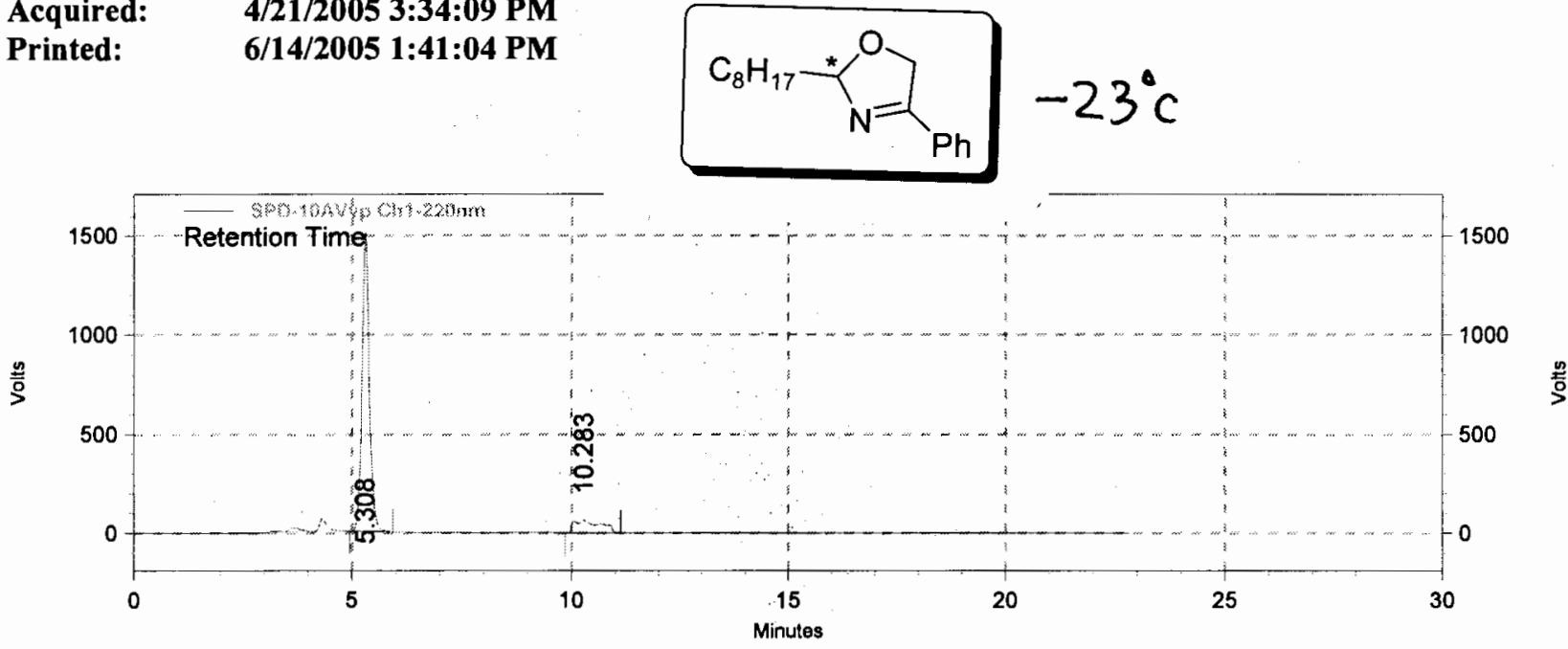

SPD-10AVvp

Ch1-220nm

Results

Retention Time

5.308

Area . Area \%

17439436

2673573

86.71

13.29

Height

Height \%

10.283

20113009

100.00

1577247

100.00 


\section{Area \% Report}
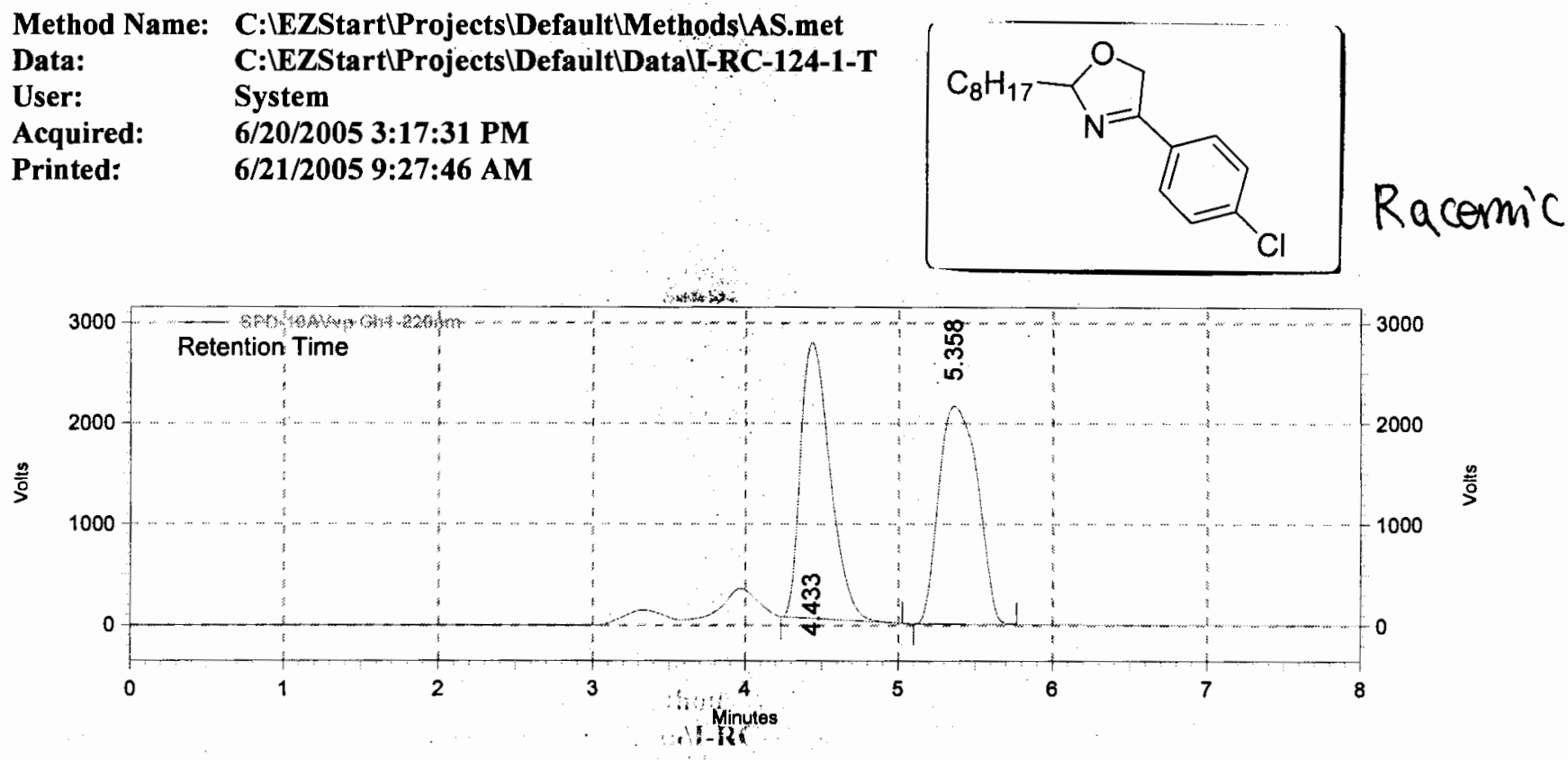

\section{SPD-10AVvp}

Ch1-220nm

Results

Retention Time

Area

Area \%

Height

Height \%

4.433

36907323

49.40

2737503

55.91

5.358

37803562

50.60

2158846

44.09

\begin{tabular}{|r|r|r|r|r|}
\hline Totals & 74710885 & 100.00 & 4896349 & 100.00 \\
\hline
\end{tabular}




\section{Area \% Report}

Page 1 of 1

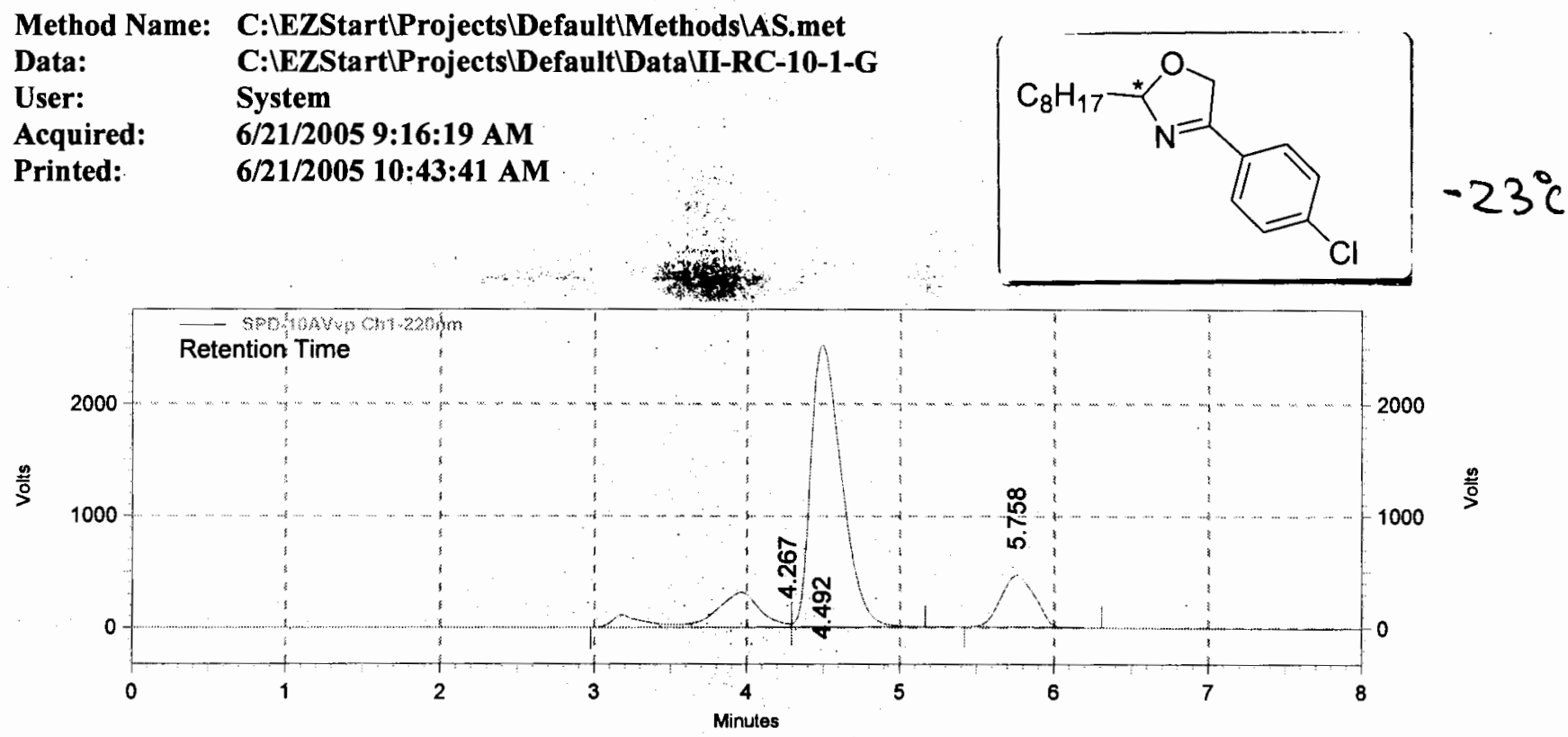

SPD-10AVvp

Ch1-220nm

Results

Retention Time

4.267

Area

Area $\%$

Height

Height \%

4.492

7604678

15.20

70.85

30510

1.01

5.758

6979623

13.95

465523

15.41

Totals 